\title{
An Analysis Tool for the Conceptual Design of High-Lift Systems
}

\author{
by \\ William J.M. Bissonnette \\ Bachelor of Engineering, Ryerson University, 2014 \\ A thesis
presented to Ryerson University \\ in partial fulfillment of the \\ requirements for the degree of \\ Master of Applied Science \\ in the Program of \\ Aerospace Engineering
}

Toronto, Ontario, Canada, 2016

(C)William J.M. Bissonnette 2016 
I hereby declare that I am the sole author of this thesis. This is a true copy of the thesis, including any required final revisions, as accepted by my examiners.

I authorize Ryerson University to lend this thesis to other institutions or individuals for the purpose of scholarly research

I further authorize Ryerson University to reproduce this thesis by photocopying or by other means, in total or in part, at the request of other institutions or individuals for the purpose of scholarly research.

I understand that my thesis may be made electronically available to the public. 


\title{
An Analysis Tool for the Conceptual Design of High-Lift Systems
}

\section{William J.M. Bissonnette}

Master of Applied Science, Aerospace Engineering, Ryerson University, Toronto 2016

\begin{abstract}
An aerodynamic analysis tool for the conceptual design of high-lift devices has been developed. The method employs a higher-order potential flow method that uses elements of distributed vorticity. The subsequent numerically robust model allows for strong wake interactions, even when using a relaxed wake. The method predicts lift and induced drag values that compare well with multiple data experiments, and, when implemented in a panel code, maximum lift predictions of a high-lift system are found with an error of $6 \%$ from experimental data. This method is used to assess the impact that various wake models have on lift and induced drag predictions. This study shows that significant errors can be introduced when employing a prescribed wake model set to extreme angles. Compared to an approach using CFD, the computational expense of these models is relatively low. A single analysis requires minutes, making these models suitable for the iterative conceptual design phase.
\end{abstract}




\section{Contents}

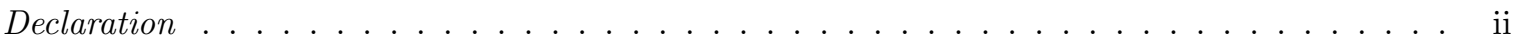

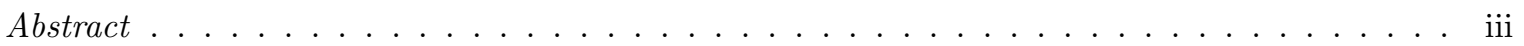

List of Tables . . . . . . . . . . . . . . . . . . . . . . . vi

List of Figures . . . . . . . . . . . . . . . . . . . . vii

List of Symbols . . . . . . . . . . . . . . . . . . . . . . . . . xi

1 Introduction $\quad 1$

1.1 High-Lift Devices . . . . . . . . . . . . . . . . . . . . . . 1

1.2 High-Lift Analysis Methods: A Brief History . . . . . . . . . . . . . . . . . . 4

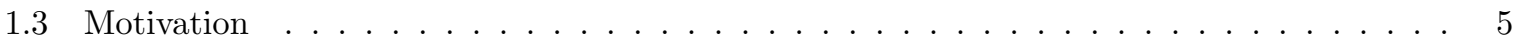

2 High-Lift Analysis $\quad 6$

2.1 Conceptual Design Analysis . . . . . . . . . . . . . . . . . . . . 6

2.2 Computational Fluid Dynamics . . . . . . . . . . . . . . . . . . . . . 10

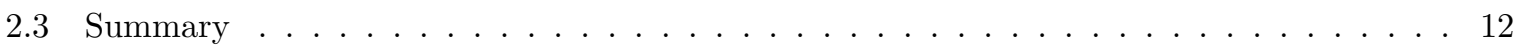

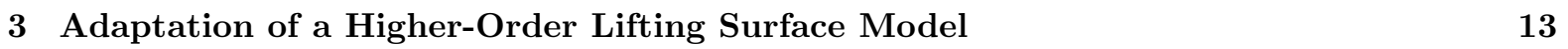

3.1 Implementation . . . . . . . . . . . . . . . . . . . . . . . . . . . 13

3.2 Comparison to Experimental Data $\ldots \ldots \ldots \ldots \ldots \ldots \ldots \ldots$

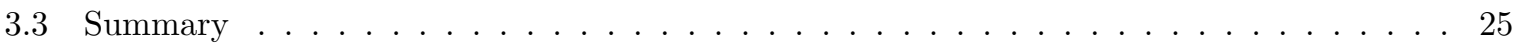

4 Extension to a Panel Method $\quad 26$

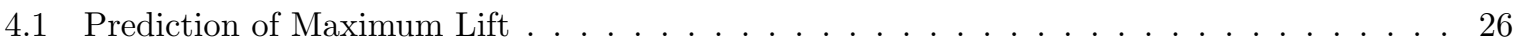

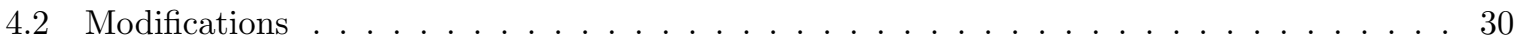


4.3 Surface Pressures . . . . . . . . . . . . . . . . . . . . . . . 32

4.4 Example Application . . . . . . . . . . . . . . . . . . . . . . . . . 40

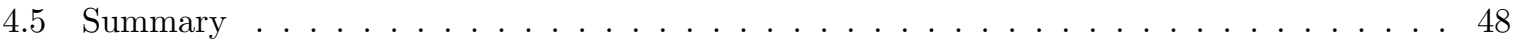

5 Analysis of Wake Shapes $\quad 49$

5.1 Singularity Issues $\ldots \ldots \ldots \ldots \ldots \ldots$

5.2 Wake Induced Velocities . . . . . . . . . . . . . . . . . . . . . 53

5.3 Comparison of Wake Shapes . . . . . . . . . . . . . . . . . . . . . . . . 54

5.3 .1 Single Rectangular Wing . . . . . . . . . . . . . . . . . . 54

5.3 .2 NASA Trap Wing . . . . . . . . . . . . . . . . . . . 58

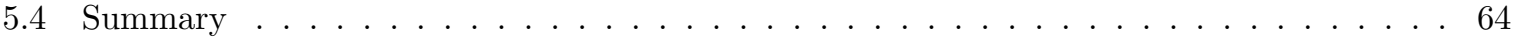

6 Conclusions $\quad 66$

6.1 Lifting Surface Method . . . . . . . . . . . . . . . . . . 66

6.2 Panel Method . . . . . . . . . . . . . . . . . . . . . . . . . 67

6.3 Wake Shapes . . . . . . . . . . . . . . . . . . . . . 67

$\begin{array}{ll}\text { Bibliography } & 68\end{array}$ 


\section{List of Tables}

2.1 Computation times for the NASA Trap Wing using CFD Methods. . . . . . . . . . . . . 12

3.1 Analyzed NASA Trap Wing configurations from the LaRC 14x22ft Entry. . . . . . . . . . 18 


\section{List of Figures}

1.1 Two-dimensional view of a wing with a slat and single slotted flap. . . . . . . . . . . 2

1.2 Lift-to-drag ratios of a transport aircraft at four flap deflection angles. . . . . . . . . . . 3

2.1 NASA Trap Wing in the NASA Ames 12 foot pressure wind-tunnel. . . . . . . . . . . . 11

3.1 A distributed vorticity element's composition. . . . . . . . . . . . . . . . . . . . 14

3.2 Distributed vorticity elements placed along the camber plane of a rectangular wing and the relaxed wake shed from the trailing edge. . . . . . . . . . . . . . 15

3.3 Rectangular wing with a 45 degree twist constructed using distributed vorticity elements. 16

3.4 Distributed vorticity elements placed along the approximate camber plane of the NASA Trap Wing LaRC Config. 1. . . . . . . . . . . . . . . . . . . . 17

3.5 NASA Trap Wing camber models. . . . . . . . . . . . . . . . . . . . . 17

3.6 NASA Trap Wing slat camber models. . . . . . . . . . . . . . . . . . . . 18

3.7 NASA Trap Wing LaRC Config. 1 wake plot after 20 timesteps at an angle of attack of 15 degrees. . . . . . . . . . . . . . . . . . . . . . . . . . . 19

3.8 NASA Trap Wing LaRC Config. 1 wake plot after 20 timesteps at an angle of attack of 15 degrees, as viewed from downstream looking towards the trailing edge. . . . . . . . . 19

3.9 Convergence of the predicted span efficiency factor of the NASA Trap Wing LaRC Config.

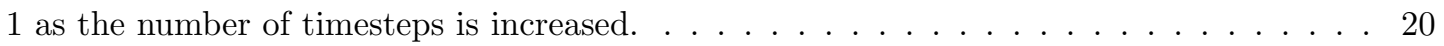

3.10 Computation time required for a single angle of attack analysis of the NASA Trap Wing LaRC Config. 1 as the number of timesteps is increased. Results were found on a personal computer using an Intel Core i7 processor capable of running at $2.9 \mathrm{GHz} . \quad \ldots . . \ldots . .21$ 
3.11 Convergence of the span efficiency factor as the number of elements is increased for the NASA Trap Wing LaRC Config. 1 at an angle of attack of 10 degrees. . . . . . . . . . . 22

3.12 Effect of the number of elements on the computation time for a single angle of attack analysis using an Intel Core i7 processor capable of running at $2.9 \mathrm{GHz} . ~ \ldots . . \ldots .23$

3.13 Aerodynamic predictions of the NASA Trap Wing LaRC Config 1. Flap deflected 25

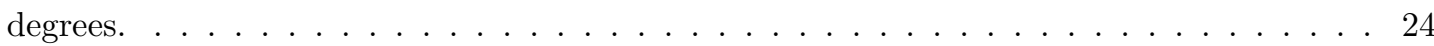

3.14 Aerodynamic predictions of the NASA Trap Wing LaRC Config. 8. Flap deflected 20

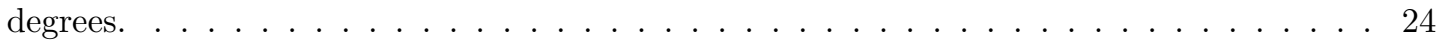

3.15 Total, induced, and profile drag of the NASA Trap Wing LaRC Config. 1. . . . . . . . . . 25

4.1 The pressure difference rule for maximum lift as a function of Mach number and Reynolds

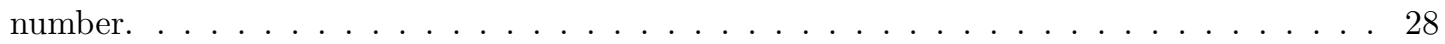

4.2 Comparison of wind tunnel measurements to panel code predictions for the RAE wing with flaps and slats. Maximum lift is predicted using the pressure difference rule. . . . . . 29

4.3 Method of defining panel geometry, beginning with the lifting surface method. . . . . . 30

4.4 Implementation of the Kutta condition with a panel method. . . . . . . . . . . . . 31

4.5 Analysis of pressures and velocities near the half span of a non-lifting cylinder of aspect ratio 20 exposed to a freestream flow of $1 \mathrm{~m} / \mathrm{s} \ldots \ldots \ldots \ldots \ldots$

4.6 Velocities along a vertical line through the center of a non-lifting wing. The dotted lines represent the top and bottom of the wing. The inset figures show the locations of the velocity profiles on the airfoil cross sections. $\ldots \ldots \ldots \ldots \ldots \ldots$

4.7 Predicted internal velocities due to the influence of the upper and lower surfaces. . . . . 37

4.8 Convergence of surface pressure coefficients near the half span of a non-lifting rectangular wing with an aspect ratio of 20 , as the number of chordwise elements, $m$, is increased

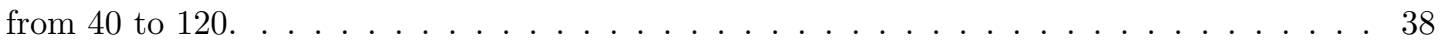

4.9 Convergence of surface pressure coefficients near the half span of a lifting rectangular wing with an aspect ratio of 20 , as the number of timesteps is increased from 10 to $100 . \quad \ldots 38$

4.10 Maximum lift angle of attack as predicted by the pressure difference rule. . . . . . . . . 39

4.11 The NASA Trap Wing LaRC Config. 1 surfaces modeled using triangular elements. . . . 40

4.12 NASA Trap Wing Config. 1 airfoil approximations. . . . . . . . . . . . . . . . . . . 41 
4.13 Aerodynamic predictions of the NASA Trap Wing LaRC Config. 1 using a panel method with a fixed wake. . . . . . . . . . . . . . . . . . . . . . . 42

4.14 Simplified multiple lifting element system modeled with a panel code. This figure shows the wake from the slat becoming fixed inside the main wing. . . . . . . . . . . . . . . . 43

4.15 The relaxed wake shed by the slat of the NASA Trap Wing modeled with a panel method at an angle of attack of 12 degrees. . . . . . . . . . . . . . . . . . . . . . . . 44

4.16 Aerodynamic predictions of the NASA Trap Wing LaRC Config. 1 using a panel method with a relaxed wake. . . . . . . . . . . . . . . . . . . . . . . 45

4.17 Predictions of the difference between the minimum pressure peak and trailing edge pressure in the spanwise direction for the NASA Trap Wing LaRC Config. 1. Each spanwise section corresponds to $10 \%$ of the halfspan. . . . . . . . . . . . . . . . . . . 46

4.18 Prediction of the minimum pressure peak value in the spanwise direction for the NASA Trap Wing LaRC Config. 1. Each spanwise section corresponds to $10 \%$ of the halfspan. . 47

4.19 Surface pressure predictions of the NASA Trap Wing LaRC Config. 1 at an angle of attack of 24 degrees using a modified relaxed wake. . . . . . . . . . . . . . . . . . . . . 48

5.1 A wing and trailing-edge flap system modeled using a traditional vortex lattice method. The discrete filaments used to model the wake shed from the main wing pass closely to the flap, resulting in large velocities at the control point locations. . . . . . . . . . . . 50

5.2 High-Lift system modeling simplifications. . . . . . . . . . . . . . . . . . . . . . 51

5.3 The velocities induced by the wake of a traditional vortex lattice method, as well as a higher-order continuous vorticity method, along three lines on a plane perpendicular to the wake. The lower inset shows the location of the plane where the velocities were found. 52

5.4 Effect of prescribed wake geometries on the aerodynamic predictions of a cambered rectangular wing using a panel method. . . . . . . . . . . . . . . . . . . . 53

5.5 Two prescribed wake models used to analyze a canard-wing fighter concept at an angle of attack of 10 degrees. . . . . . . . . . . . . . . . . . . . . . . . . . 54

5.6 Wake shapes analyzed on a rectangular wing with an aspect ratio of 10 at an angle of attack of 6 degrees. . . . . . . . . . . . . . . . . . . . . 56 
5.7 Comparison of lift and induced drag for five different wake shapes for a rectangular wing

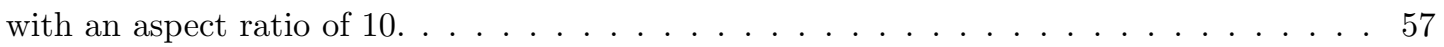

5.8 Effect on incoming flow velocity due to three prescribed wake shapes of equal strength. Wake 1 is aligned with the freestream (drag free), wake 2 is prescribed above the freestream (positive), and wake 3 below the freestream (negative). The lower inset shows the additional component of the resultant flow vector in the freestream direction as a wake is

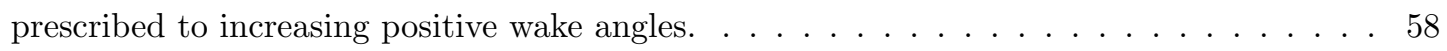

5.9 Wake shapes analyzed on the NASA Trap Wing LaRC Config. 1 at an angle of attack of

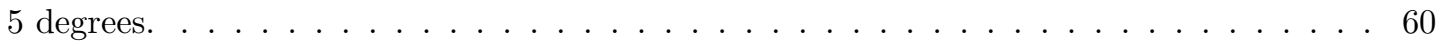

5.10 Drag predictions for the NASA Trap Wing LaRC Config. 1 using different wake types. . . 61

5.11 Lift predictions for the NASA Trap Wing LaRC Config. 1 using different wake types. . . 62

5.12 Corrected lift predictions. Values are corrected based on the conclusion that lift coefficients deviate $2.7 \%$ for every 10 degree difference between the prescribed wake angle and

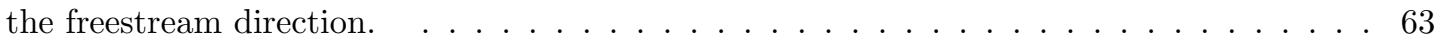

5.13 Predicted spanwise circulation distributions for the NASA Trap Wing LaRC Config. 1, at an angle of attack of 20 degrees. Values are normalized to the circulation at the main wing root section, as predicted with a relaxed wake. . . . . . . . . . . . 64 


\title{
List of Symbols
}

\author{
$\eta \quad$ local spanwise coordinate \\ $\Gamma \quad$ circulation \\ $\gamma \quad$ vorticity \\ $\gamma_{s p} \quad$ ratio of specific heats \\ $\rho \quad$ density \\ $\xi \quad$ local chordwise coordinate \\ $A, B, C$ circulation coefficients \\ b wingspan \\ c section chord length \\ $C_{D} \quad$ three-dimensional drag coefficient \\ $C_{L} \quad$ three-dimensional lift coefficient \\ $c_{l} \quad$ two-dimensional section lift coefficient \\ $c_{n} \quad$ two-dimensional section normal force coefficient \\ $C_{P} \quad$ local pressure coefficient \\ $C_{D i} \quad$ induced drag coefficient \\ $C_{D p} \quad$ three-dimensional profile drag coefficient
}




\begin{tabular}{|c|c|}
\hline$C_{L, \max }$ & maximum lift coefficient \\
\hline$C_{P, \min }$ & minimum pressure coefficient \\
\hline$C_{P, T E}$ & trailing edge pressure coefficient \\
\hline$C_{P}^{*}$ & critical pressure coefficient \\
\hline$D_{i n d}$ & induced drag force \\
\hline$e$ & span efficiency \\
\hline$g$ & local flap gap distance \\
\hline$L^{\prime}$ & section lift force \\
\hline$M$ & local Mach number \\
\hline$m$ & number of chordwise elements \\
\hline$M_{\infty}$ & freestream Mach number \\
\hline$n$ & number of spanwise elements \\
\hline$N^{\prime}$ & section normal force \\
\hline$p$ & local static pressure \\
\hline$p_{\infty}$ & freestream static pressure \\
\hline$u$ & streamwise velocity \\
\hline$V$ & velocity \\
\hline$v$ & spanwise velocity \\
\hline$V_{\infty}$ & freestream velocity \\
\hline$w$ & downwash velocity \\
\hline CFD & Computational Fluid Dynamic \\
\hline
\end{tabular}

CPU Central Processing Unit 
DVE Distributed Vorticity Element

PDR Pressure Difference Rule

RANS Reynolds Averaged Navier-Stokes 


\section{Chapter 1}

\section{Introduction}

\section{$1.1 \quad$ High-Lift Devices}

To maximize their overall efficiency, commercial transport aircraft must be able to adapt to a variety of flight requirements. Rudolph shows that often, commercial aircraft make use of slats and slotted flaps to achieve the required performance at low airspeeds [1]. An example of a wing section with a slat and single slotted flap is shown in Fig. 1.1. Historically, the main design considerations of these highlift devices were driven by the airfield performance that requires the aircraft to fly slow, maintain safe approach speeds, and achieve minimum climb rates without negative effects on the cruise performance $[2,3,4]$. More recently, designers have been focusing on reducing the weight and complexity of the highlift systems, which are a significant factor in the total cost of the entire aircraft [2]. Rudolph estimates that a single slat and double slotted flap system can total between $6 \%$ and $11 \%$ of the production cost; this estimate increases as more complex systems are used [1,2]. Van Dam describes that the high costs stem from the time consuming process needed to design and test such devices, since the flows, geometry, actuation, and supports are complex, heavy, and maintenance intensive [2].

Small changes in the design of these devices can result in large performance gains. If designed properly, the benefits of such devices are clear. The following performance gains for a generic largetwin engine commercial aircraft were given by Meredith [6]. 1) A $1.5 \%$ increase in the maximum lift coefficient equates to a $3000 \mathrm{~kg}$ (6600 lbs) increase in payload, corresponding to 33 extra passengers. 2) A $1 \%$ increase in the takeoff lift-to-drag ratio is equivalent to a $1270 \mathrm{~kg}$ (2800 lbs, 14 passengers) 


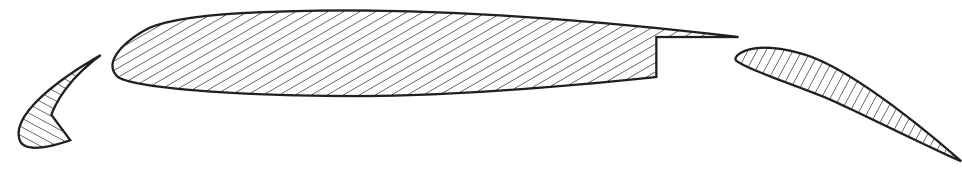

Figure 1.1: Two-dimensional view of a wing with a slat and single slotted flap [5].

increase in payload, or a $278 \mathrm{~km}(150 \mathrm{~nm})$ increase in range. 3) A 0.1 increase in the lift coefficient at a constant angle of attack is equivalent to reducing the approach attitude by about 1 degree. Ignoring the takeoff needs, this could allow shortening of the landing gear by approximately $35.5 \mathrm{~cm}$ (14 in) for a given tail strike attitude angle used and a decrease in structural weight by $635 \mathrm{~kg}$ (1400 lbs).

Specifically, the use of high-lift devices are necessary in order to achieve these performance gains since they allow aircraft to takeoff and land on runways of acceptable length without penalizing the cruise efficiency [2]. Van Dam showed that, compared to a simple wing, a wing with a retractable high-lift device can have an $18 \%$ higher cruise lift-to-drag ratio while maintaining a similar maximum lift and minimum drag for takeoff. This performance advantage during cruise is primarily due to the smaller wing area that is possible with high-lift devices.

In the early 1970s, Smith outlined the five underlying principals of slotted high-lift devices [7]. He described that slats primarily reduce pressure peaks on the downstream elements and aid in the attachment of flow over the beginning of the main wing. This so called slat effect allows higher angles of attack to be achieved without flow separation occurring. Flaps work by increasing the wing's camber and surface area. The presence of a slotted flap increases the angle of attack at the trailing edge of the upstream element, which requires an increase in circulation of the upstream element in order to satisfy the Kutta condition on the upstream element. This circulation effect increases lift on the upstream element, but also increases pressure recovery demands. Since a gap is formed with a slat or slotted flap, the dumping effect describes that the flow from the upper-surface of the forward element is discharged into a higher velocity region, reducing the pressure recovery demands. Therefore, an off-surface pressure recovery occurs as the deceleration of the flow is done without wall contact. Finally, according to Smith, each element in a multi-element system starts with a fresh boundary layer. Since this layer is thin, it can support greater positive pressure gradients without separation.

Knowing the mechanisms of how slotted devices work allows designers to analyze high-lift devices more accurately, and therefore, design more efficient systems. As a result, the design requirements 
of high-lift systems are increasingly becoming more complex. Today, high-lift wing design is highly multidisciplinary and includes structural and subsystem designs. A challenging factor of the high-lift design is that the development process usually starts with the cruise wing design $[8,9,10]$. Unfortunately, the sensitive nature of high-lift devices means that small changes to the cruise wing can render the highlift devices useless for the new configuration [4]. This can present a conflict as aircraft systems become more complex, timelines are shortened, and budgets are reduced.

The design of high-lift systems is largely based on the takeoff performance requirements as governed by regulatory authorities such as the Federal Aviation Administration [11]. The takeoff and initial climb segments are governed by an aircraft's stall speed, and therefore the maximum lift coefficient of the airframe. Upon entering the second segment climb, the optimum climb gradient depends on the aircraft's lift-to-drag ratio. This presents a design challenge, since, as it is shown in Fig. 1.2, a downward flap deflection on a transport aircraft increases the maximum lift coefficient while it also decreases the lift-to-drag ratio. As a result, the entire takeoff performance is a balance of the lift and drag forces [3].

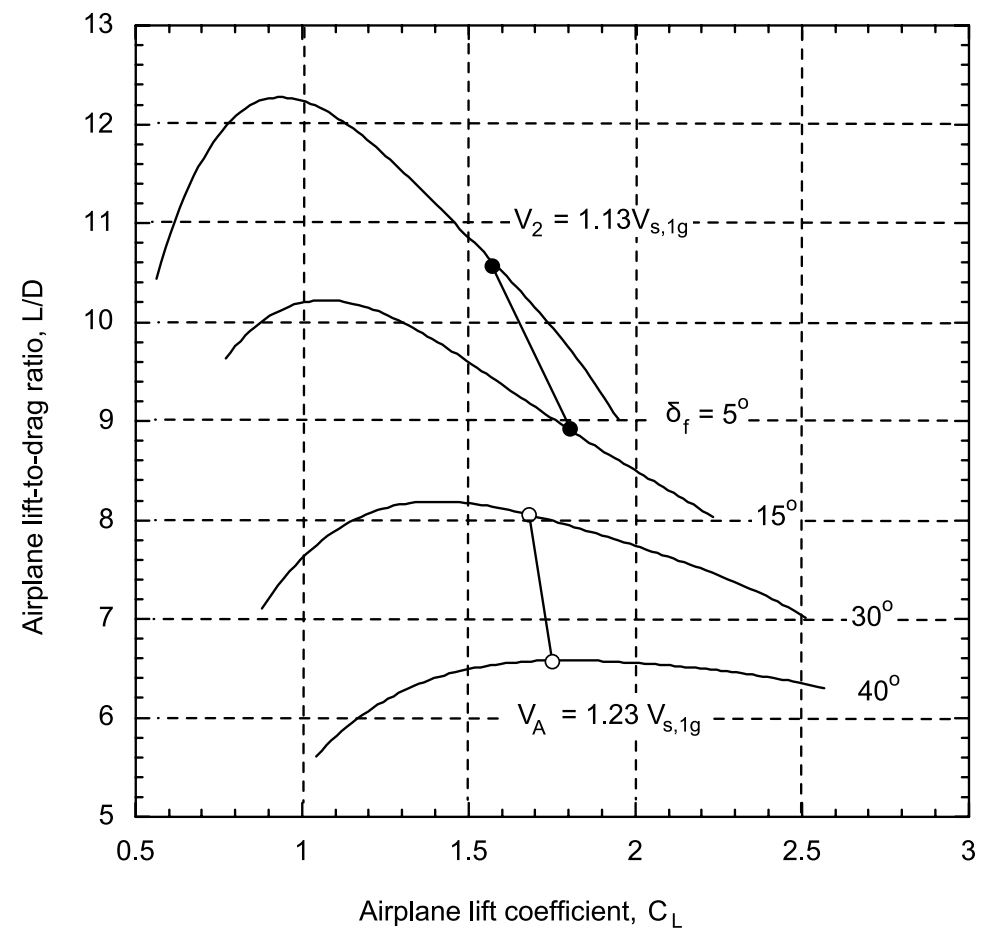

Figure 1.2: Lift-to-drag ratios of a transport aircraft at four flap deflection angles [2].

To solve this, the most efficient design must come from an iterative design process that incorporates 
not only the main wing design, but also takes a multidisciplinary optimization approach [8]. It is therefore essential to have a method that quickly and accurately analyzes the aerodynamic performance of high-lift devices early in the conceptual and preliminary design phases $[4,8]$. The method introduced herein has the potential to provide such an aerodynamic analysis tool.

\subsection{High-Lift Analysis Methods: A Brief History}

The computational analysis of high-lift systems was traditionally performed using inviscid methods [2]. These numerical methods often are used to formulate an inviscid solution using a potential flow model that represents lifting surfaces. A limitation of these methods is their underlying assumption of irrotational flow that neglects any viscous effects. Therefore, these methods cannot predict profile drag and flow separation, unless they are coupled with a viscous solver. Nonetheless, methods such as these can provide good performance predictions and have a computational efficiency advantage over finite difference methods (computational fluid dynamics) since the solution has less unknowns and requires solving for the strength of singularity elements instead of solving for the flowfield in the entire flow volume $[12,13]$.

For two-dimensional high-lift airfoil analysis, viscid/inviscid coupled methods tend to be less robust than finite difference methods that solve the two-dimensional Navier-Stokes equations with the use of Reynolds Averaged Navier-Stokes (RANS) solvers [2]. Due to the singular nature of the low-order numerical elements, problems with geometry modeling can occur. Advances in computational power have allowed many finite difference methods to be developed in the recent past, each tuned for specific applications. The accurate and consistent prediction of maximum lift, however, continues to be a problem for RANS methods $[2,14,15]$.

Less progress has been made in three-dimensional RANS analysis tools for aircraft with high-lift systems. These tools can be used to accurately analyze complicated systems, although the time required to achieve such solutions is often in the order of days [2]. This is too long for most conceptual design problems. Because of this computational expense, semi-empirical methods and inviscid methods such as lifting surface methods and panel codes, with and without viscous coupling, are still commonly used during the early design stages today. In fact, these methods were used at the conceptual design stage of many modern commercial aircraft including the Airbus A380, Airbus A350, and Boeing 777, before 
being validated with three-dimensional RANS solvers and wind-tunnel testing in later design phases $[4,8,10,16]$.

One of the drawbacks of potential flow methods is that, even when coupled to a viscous solver, they often cannot predict the maximum lift coefficient of a lifting system. As a result, these methods are largely used to determine performance deltas due to specific configuration changes, with wind-tunnel testing determining the baseline values. When high angle of attack analyses are necessary, semi-empirical methods and theoretical limits are often used at the conceptual design stage [7, 17].

\subsection{Motivation}

This thesis describes a method that analyzes high-lift devices at the conceptual design stage. The method has the efficiency advantages of potential flow models without many of the associated issues related to common low-order methods. This is due to the use of elements of distributed vorticity representing the lifting surfaces, rather than discrete vortex filaments. The velocity fields induced by these elements largely avoid any velocity peaks without the need for corrective measures, such as solid-core models. As a result, it avoids many of the numerical issues often encountered with more conventional potential flow methods, even when employing a relaxed-wake model and modeling several, closely interacting lifting surfaces.

The method is employed first as a lifting surface method, and aerodynamic predictions are compared to wind-tunnel data for multiple high-lift configurations. The method is then extended to a full panel code that determines surface pressures with the goal of estimating the maximum lift capabilities of the high-lift systems. These tools allow for an efficient analysis of high-lift devices at the conceptual design stage.

Ultimately, the robust nature of the higher-order method that was used for these models allows for analyses when strong interaction occurs between lifting surfaces, and where lower-order methods may suffer. The higher-order method allows for the automatic generation and relaxation of wake elements, a procedure that is often not performed with high-lift devices at the conceptual design stage due to the numerical issues typical for the more traditional methods. To determine the effects of prescribed wakes, a study was performed on the effects of wake positions on the aerodynamic predictions of high-lift devices. 


\section{Chapter 2}

\section{High-Lift Analysis}

Optimization studies performed during the conceptual design stages require computationally fast analysis tools capable of efficiently modeling and analyzing geometries. Historically, the three-dimensional analysis of high-lift systems was limited to lifting surface models and wind-tunnel testing. Complex shapes simply could not be modeled or analyzed efficiently using more advanced theoretical based methods. More recently, advancements in computational efficiency have allowed the use of advanced three-dimensional potential flow models alongside three-dimensional Euler solvers and two-dimensional Reynolds Averaged Navier-Stokes solvers to optimize high-lift devices during the preliminary design stages.

\subsection{Conceptual Design Analysis}

The potential flow lifting surface models and three-dimensional panel codes that are used during the conceptual design stages use singularity elements, typically formed with combinations of sources, sinks, and/or vorticies, to model a body exposed to a flow field $[2,8,10,16]$. To satisfy Kutta condition, vorticity must be shed from these lifting surfaces, which represents the shear layer in the wake. Drela describes that modeling low speed flow-fields with this method is attractive for a number of reasons, including [13]:

- In flow with thin viscous layers, very little accuracy is lost when the vorticity is lumped into vortex sheets and placed on body and wake surfaces, which is equivalent to an inviscid-flow approximation 
- Only body surfaces and possibly wakes have to be defined, whereas finite difference methods require an entire grid to be constructed throughout the flowfield

- Numerical panel methods, which employ the sheet representation, require roughly $1 / 100$ fewer unknowns than corresponding grid methods for a given level of accuracy

- In areas where the velocity or potential jump across a sheet is known, the sheet strength can be computed immediately.

The strengths of the singularity elements are not unique and must be determined via boundary conditions to satisfy the number of unknowns. This can be approached in one of two ways. The direct, Neumann method forces flow to be tangential to the surface by setting the normal flow velocity component equal to zero at control points on the surface. This simple condition is relatively easy to implement and is used with many lifting surface methods. The Dirichlet boundary condition solves for the surface element strengths necessary to enforce the velocity potential at control points, indirectly setting the velocities [12]. Often, the Dirichlet boundary condition is used to set a constant potential inside a body, thereby forcing the flow to have zero velocity. As such, it can only be used when thickness effects are modeled with a full panel code.

These boundary conditions provide enough equations to solve for flow about non-lifting bodies. However, for lifting cases, the amount of circulation is still not unique. To solve this, the Kutta condition is imposed along the trailing edge with the addition of a wake that models the shear layer coming off the trailing edge. The vorticity shed at this location defines the strength of the wake, which contributes to the induced velocities used to determine surface singularity strengths. Most low-order potential flow models use vortex filaments in order to represent the wake $[18,19]$. Their strength depends on the spanwise changes of the bound circulation. In the streamwise direction, their strength remains constant under steady conditions since a wake, unlike surfaces, cannot support any forces.

The shape of the wake can have impacts on the overall solution, and must be carefully considered [12]. Commonly used is the drag-free wake model, in which the wake-vortex filaments are aligned with the freestream direction. Other wake models use prescribed wake shapes to avoid any adverse numerical interaction of the wake filaments, for example, with downstream lifting surfaces. Nevertheless, prescribed wake shapes are not force-free and can result in inaccuracies of the predicted aerodynamic forces. In contrast to a drag-free wake, in a relaxed wake model the wake filaments are aligned with the local 
velocity field leading to a force-free wake. Relaxed wake models are able to capture second-order wake effects cased by the wake roll-up, which is significant if there is strong interaction between lifting surfaces and their wakes. Disadvantages of a relaxed wake model are an increase in computational expense due to the relaxation process, possible numerical issues related to the singular nature of the wake filaments, and challenges to determine induced drag using traditional approaches.

Potential flow methods with fixed and relaxed wake models can develop numerical issues related to the large velocities near the center of the vortex filaments that are used to model the shear layer in the wake. The large velocities, which are nonphysical, can cause adverse interference effects, for example, when analyzing multiple lifting surfaces or during the relaxation process. Although solid-core models can alleviate the issues of large velocities, they still result in a velocity distribution with local peaks that are nonphysical. Subsequently, the choice of core size can drive the solution.

After the addition of a wake to the solution, the singularity strength distribution across the lifting surface can be used to determine the lift it generates. This can be done in a number of ways. The first involves determining the surface velocities and performing a numerical integration of the vertical component of the surface pressures over the body. Of course, this requires a full panel code to determine upper and lower surface pressures. A second method to determine lift is by integrating the sectional lift at multiple spanwise locations across a surface. The section lift force, $\overrightarrow{L^{\prime}}$, can be found by applying the Kutta-Joukowsky theorem:

$$
\overrightarrow{L^{\prime}}=\left(\overrightarrow{V_{\infty}} \times \vec{\Gamma}\right) \rho
$$

where the circulation, $\vec{\Gamma}$, is perpendicular to the freestream velocity, $\overrightarrow{V_{\infty}}$, and is found knowing the surface singularity distribution. This method has the advantage that it can be used on lifting surfaces in addition to panel codes that model thickness.

Solving for the drag forces is more difficult than the solution for lift. Numerically, d'Alembert's paradox constitutes that a two-dimensional inviscid and incompressible solution has zero pressure drag, since the streamwise pressure contributions cancel. In three dimensions, the cancellation is not total, and results in a pressure drag force, which is the induced drag of the system. When integrating the pressure forces across the entire wing surface, the danger is that small errors in the surface pressures or with the integration can become very large when compared with the small pressure drag [13]. As 
a result, surface-pressure integrations usually require a large number of panels in order to sufficiently resolve the pressure changes, especially around the leading edge.

Induced drag is more commonly found via a Trefftz plane analysis. In the Trefftz plane, which is selected far downstream from the lifting surface and normal to the freestream, the influence of the surface bound vorticity is negligible and the wake shape is assumed to be constant in the streamwise direction $[12,13]$. As a result, the problem is decomposed into a two-dimensional integration of the wake induced velocities in the Trefftz plane, $S_{T}$, according to:

$$
D_{\text {ind }}=\frac{\rho}{2} \int_{S_{T}}\left(v^{2}+w^{2}\right) d y d z
$$

where $v$ and $w$ are the velocities induced in the plane of the wake and normal to the wake, respectively, and the freestream density is represented as $\rho$.

The Trefftz plane assumption that the wake shape is constant presents difficulties when a wake relaxation routine is used. To solve this, induced drag can be predicted by applying the Kutta-Joukowski theorem, Eq. 2.1, along the trailing edge of the wing using the wake induced velocities $[20,21]$. The induced drag is the streamwise component of the resulting force. This method can be used to find the induced drag since all of the vorticity produced by the lifting surface is shed along the trailing edge, and, under steady-state conditions, the wake itself does not support any streamwise changes in vorticity. In addition to being less sensitive to wake shapes than the Trefftz plane method, this method has shown to be relatively insensitive to surface panel density variations [20, 22].

The simplest potential flow methods used today to model high-lift systems are lifting surface methods. Low-order lifting surfaces model wings using multiple vortex lines or the equivalent doublet sheets. The constant strength elements are distributed in the spanwise and chordwise directions along the zerolift plane or the mean camber plane and, correspondingly, result in a stepwise circulation distribution that does not capture any thickness effects. A common implementation of this method is the vortex lattice method. Vortex lattice methods are widely used during aircraft development, especially during the conceptual design phase, due to their computational efficiency, which allows the quick analysis of large numbers of configurations [13]. Limitations of the method stem from the singular nature of vortex filaments that are used to represent the bound circulation and the wake vorticity. As a result of the singularities, tangential velocities that are induced by these filaments approach an infinite value close 
to the center of such filaments. Lifting surfaces can also be implemented with higher-order elements which model lifting surface strengths as higher-order polynomials, thus providing a continuous spanwise circulation distribution [12].

Lifting surface methods can be coupled with two-dimensional data in order to add predictions of viscous effects to the inviscid solution using a strip method approach. For simple flows, two-dimensional potential flow methods coupled with viscous solvers can be used [23]; however, more complex flows require two-dimensional RANS solvers to estimate these effects. These methods are used extensively in the early design phases of modern high-lift systems $[4,8,10,16]$.

The effects of wing thickness can be included in an inviscid solution with a full panel code. These methods are an extension of the lifting surfaces described above, where both the upper and lower surface geometries are modeled [24]. The major advantage of this type of analysis is that a surface flow solution for complex high-lift systems can be rapidly obtained. Until the relatively recent advancements in computational power, these methods were the most advanced methods for modeling complex systems such as high-lift devices. Since the surfaces are modeled, entire aircraft configurations can be analyzed with these methods early in the design phase $[2,4,8]$.

As with lifting surfaces, panel methods can make use of low-order or high-order elements to model surfaces. Examples of commonly used low-order panel methods include VSAERO [18] and PMARC [19], both of which use constant strength source and doublet distributions over each surface panel. VSAERO is fully coupled to a viscous boundary layer solver, and was used extensively in the early design process of the Airbus A380 [8]. The most common commercially available higher-order panel method is PAN AIR, which uses quadratic doublets and linear sources to model surfaces $[25,26]$. Without a viscous solver, panel methods often require viscous decambering to reduce the predicted lift. For high-lift devices, this is commonly done by reducing flap deflection angles.

\subsection{Computational Fluid Dynamics}

As an alternative to potential flow based methods, computational fluid dynamics (CFD) can be used to solve the aerodynamic forces that lifting elements develop. A full Reynolds Averaged Navier-Stokes solution enables the prediction of viscous effects. If the equations are simplified to remain inviscid, the resulting formulas are known as the Euler equations. Both of these methods require the entire flow field 
to be modeled as a grid, rather than only the surface as is the case with potential flow models.

In 2010, the American Institute of Aeronautics and Astronautics (AIAA) held the first CFD HighLift Prediction Workshop (HiLiftPW-1). The conference presented the results of a NASA challenge to assess the numerical prediction capability of current-generation CFD technology for wings in high-lift configurations [27]. Participants employed modern CFD solvers in an attempt to accurately predict wind-tunnel data of the NASA Trap Wing (shown in Fig. 2.1). Many of the participants achieved results that showed good agreement with the experimentally obtained data $[28,29,30,31,32]$, however, the methods proved to be computationally expensive and time consuming. Table 2.1 below shows typical computing times of the multiple element NASA Trap Wing system for three of the participants. Many hours were needed to run a single analysis using the RANS CFD methods. This presents a challenge.

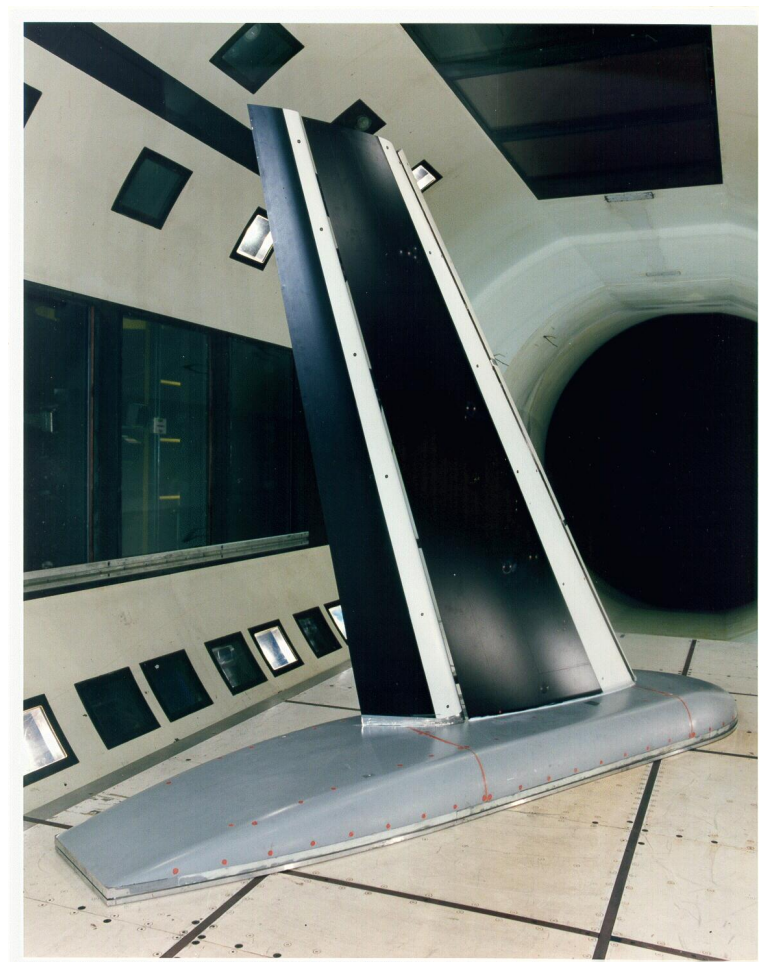

Figure 2.1: NASA Trap Wing in the NASA Ames 12 foot pressure wind-tunnel [33].

Computational fluid dynamics can attain results which agree very well with experimental data. Nevertheless, even though they may save time over wind-tunnel testing, the required computational time makes them inefficient for conceptual design and multidisciplinary optimization approaches, where multiple analyses must be run in a short amount of time. In addition, the grid generation necessary 
for CFD quite often requires manual inputs, rendering the process inefficient for an iterative design [9]. For these reasons, modern high-lift conceptual design and optimization still has limited use for three-dimensional computational fluid dynamics $[2,4,8,10,16]$.

Table 2.1: Computation times for the NASA Trap Wing using CFD Methods.

\begin{tabular}{lccc} 
Participant & Grid Points & Number of CPUs & Wall Clock Time \\
\hline Murayama, M., et al. [28] & $15-37$ million & 48 & $30-80$ hours \\
Pulliam, T., et al. [29] & 25 million & 64 & 5.8 hours \\
Sclafani, A.J., et al. [30] & 25 million & 24 & 26 hours
\end{tabular}

\subsection{Summary}

High-lift device design at the conceptual design stage requires an iterative optimization to achieve the most efficient design. The aerodynamic analysis during these studies must be able to quickly predict performance changes due to design modifications. Three-dimensional RANS methods have the ability to predict the aerodynamic forces on high-lift devices well, but the time required and the need for user-refined grid generations still renders them inefficient for iterative design studies. For this reason, potential flow methods such as lifting surface methods and full panel codes are still used today. However, many of these methods are subject to numerical issues, which must be accounted for. 


\section{Chapter 3}

\section{Adaptation of a Higher-Order Lifting Surface Model}

As mentioned, the conceptual design of modern high-lift devices incorporates potential flow models which may suffer due to numerical singularity issues. As such, a higher-order potential flow model has been adapted to predict the aerodynamic forces on high-lift devices. This method differs from conventional panel codes and vortex lattice methods in that it uses continuous vorticity instead of discrete filaments, thus it has fewer singularity issues than conventional approaches. The method provides a simple and reliable induced drag calculation, even when a relaxed wake model is used. The approach was described in Ref. 5 and is summarized in this chapter.

\subsection{Implementation}

The method is based on the multiple lifting line method developed by Horstmann [34], which divides a lifting surface into elementary wings modeled using a parabolic spanwise circulation distribution. The strength of the circulation, $\Gamma$, is defined as:

$$
\Gamma(\eta)=A+B \eta+C \eta^{2}
$$


and the shed vorticity distribution is then:

$$
\gamma(\eta)=B+2 C \eta
$$

where $\eta$ is the local spanwise coordinate.

The higher-order method employed here is an extension of this method developed by Bramesfeld and Maughmer [35]. As shown in Fig. 3.1, an element is created that consists of two elementary wings of Horstmann's multiple lifting line method. The combination of two elementary wings that have opposite strengths forms a Distributed Vorticity Element, or DVE. Subsequently, a distributed vorticity element consists of two vortex filaments that satisfy Helmholt's law in the streamwise direction (shown as the $\xi$ direction in Fig. 3.1) with a vortex sheet. These elements can be placed along the zero-lift plane or along the mean camber plane of the lifting surfaces. Accordingly, the wing circulation is represented by one or several second-order splines of the spanwise bound circulation distribution, interspersed with vortex sheets with first-order splines of spanwise vorticity distribution.

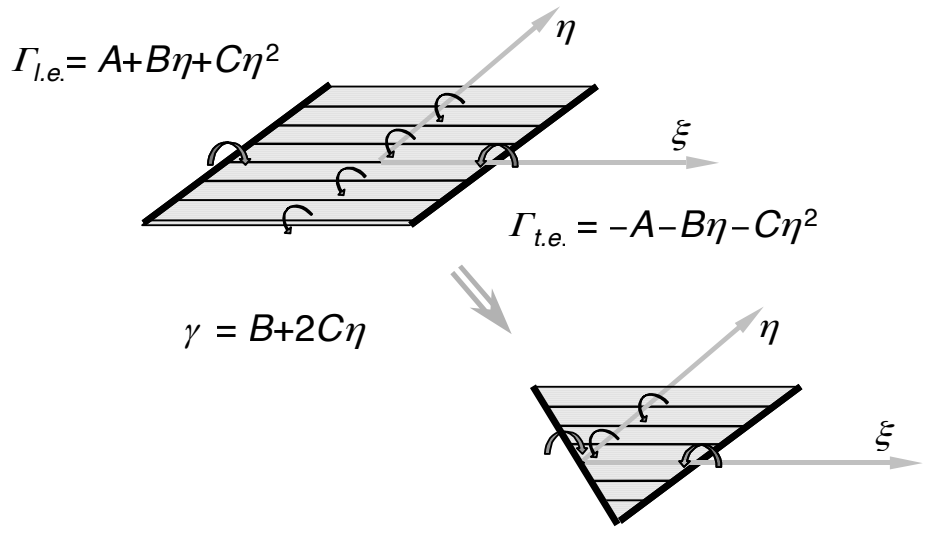

Figure 3.1: A distributed vorticity element's composition. Adapted from Ref. 35.

A time stepping method is used to emit a new spanwise row of distributed vorticity elements into the wake each timestep that the wing is moved forwards. This wake can then be fixed to a specified direction, or allowed to move with the local velocities to form a relaxed wake, as is shown in Fig. 3.2. Under steady conditions, streamwise circulation in the wake is constant and depends only on the vorticity shed into the wake along the trailing edge, therefore, the spanwise filaments in the wake are removed. 
As a result of the continuous vorticity distribution in the wake, the induced velocities in the wake are finite, therefore avoiding many of the singularity problems of traditional potential flow methods without requiring a solid core model or similar numerical corrections.

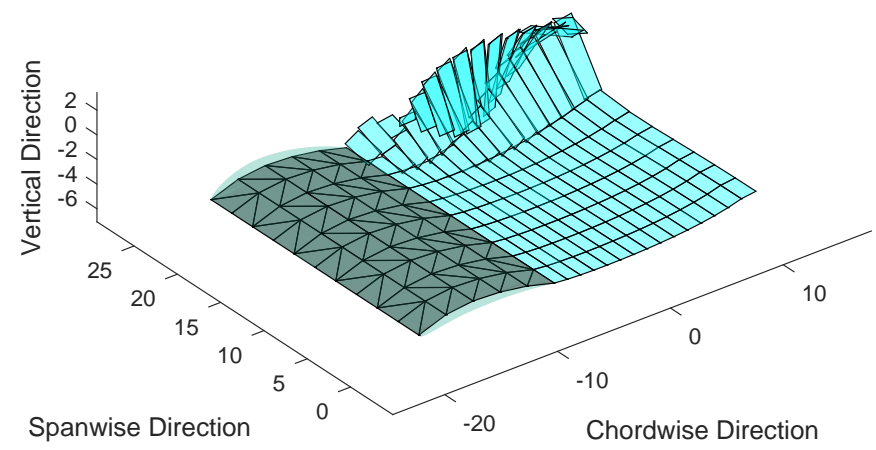

Figure 3.2: Distributed vorticity elements placed along the camber plane of a rectangular wing and the relaxed wake shed from the trailing edge [36].

The method computes lift forces along the bound circulation of the lifting lines using the KuttaJoukowski theorem, Eq. 2.1. Induced drag is computed along the trailing edge using the vorticity passed into the wake at this location, and the velocities that the wake induces along the trailing edge. A more detailed description of the method is given in Ref. 22 .

A major advantage of this approach that is implemented in the analytical tool called FreeWake, is its ability to model multiple lifting surfaces and study the interactions between them, as shown in Refs. 5 and 37. In FreeWake, trapezoidal distributed vorticity elements are placed along the zero-lift plane, essentially building the lifting surface using flat plates.

The condition that the elements must remain planar can cause gaps to form on the lifting surface if twist is introduced into the wing, as seen in Fig. 3.3a. To solve this, Combes extended the FreeWake method to model wings using triangular elements [38]. As seen in Fig. 3.1, by reducing the length of one of the sides of each element to zero, the elements become triangular. Thus, as shown in Fig. 3.3b, the method can be used to model twist distributions without the formation of gaps inbetween elements. 
The robustness of this method allows for the elements to be placed on the curved mean camber plane, rather than zero-lift plane.

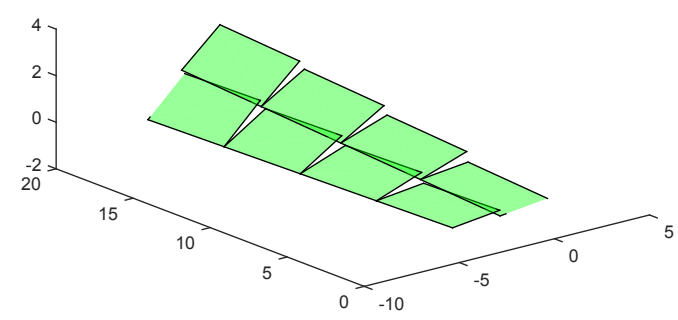

(a) Trapezoidal elements.

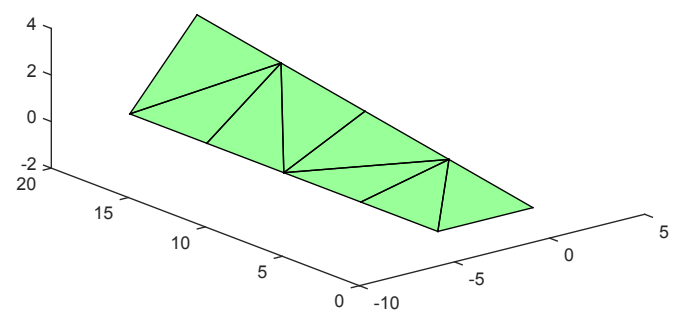

(b) Triangular elements.

Figure 3.3: Rectangular wing with a 45 degree twist constructed using distributed vorticity elements.

To model high-lift devices, a geometry input tool was developed, as described in Refs. 5 and 39. This method supports the quick modeling of complex geometries from an array of input methods to support the users needs. Modifications can also be made to already created geometries to easily support iterative optimization tasks. Finally, the geometries can be plotted to ensure they have been correctly modeled.

\subsection{Comparison to Experimental Data}

To determine the accuracy of this method, the NASA Trap Wing was used as a source of data. Using this model provides a comparison to a complex geometry set which has been analyzed by modern CFD solvers $[28,29,30,31,32]$.

Given the availability of the CAD models for multiple NASA Trap Wing configurations, the geometries were modeled with distributed vorticity elements using the developed design tools. Figure 3.4 shows the geometry for the NASA Trap Wing LaRC Full Span Config. 1 setup as imported into the tool. Note that the elements have been extended to the symmetry plane (top of image) to remove the gap made

when the slat and flap were deflected. This gap was sealed in the wind-tunnel experiment, as seen in Fig. 2.1.

The first NASA Trap Wing configuration used for the analysis was the LaRC Full Span Config. 1. This configuration has a 30 degree slat deflection and a 25 degree flap deflection. The model was input as 3 separate wings, without the body used in the NASA Trap Wing data experiment. The body, which 


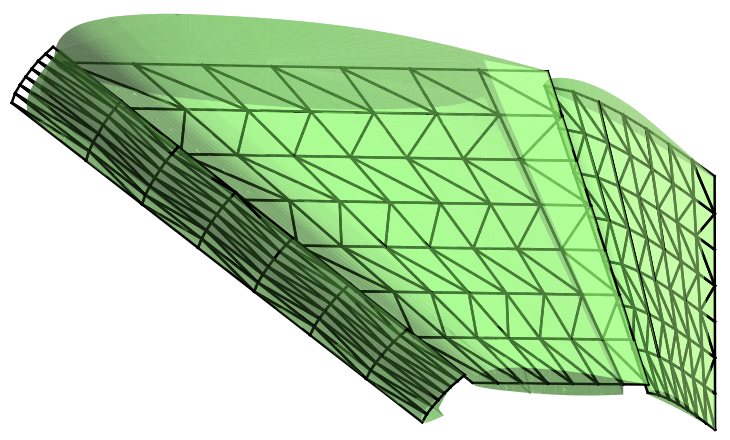

Figure 3.4: Distributed vorticity elements placed along the approximate camber plane of the NASA Trap Wing LaRC Config. 1.

can be seen at the bottom of Fig. 2.1, was removed to make the analysis less complex. The camber-line used for the flap element was approximated using the analytical tool XFOIL [23]. The camber-line for the main wing is modeled as a flat plate. Figure 3.5a shows that this is a reasonable approximation. Figure 3.5b shows the camber-line on the flap as modeled for the analysis.

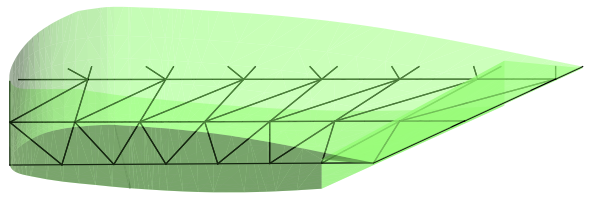

(a) Main wing.

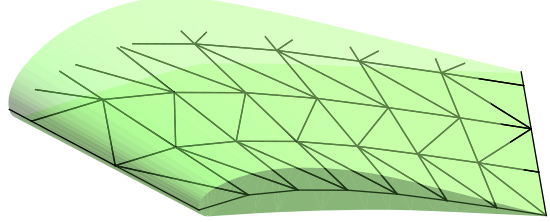

(b) Flap.

Figure 3.5: NASA Trap Wing camber models.

Estimating the camber line of complex geometries can be difficult. Two possibilities for modeling the camber line of the NASA Trap Wing slat are shown below. Because of the method used to apply camber to the panels, the camber line in Fig. 3.6a is impossible to achieve. As shown by the arrows, the camber line must be offset perpendicular to the defined chord line. The selected camber-line is shown in Fig. 3.6b.

The second NASA Trap Wing configuration analyzed was the LaRC Full Span Config. 8. This configuration reduces the flap deflection from Config. 1 by five degrees to show the effects of performance deltas due to small flap deflections. A summary of the analyzed NASA Trap Wing configurations is shown in Table 3.1 below. 


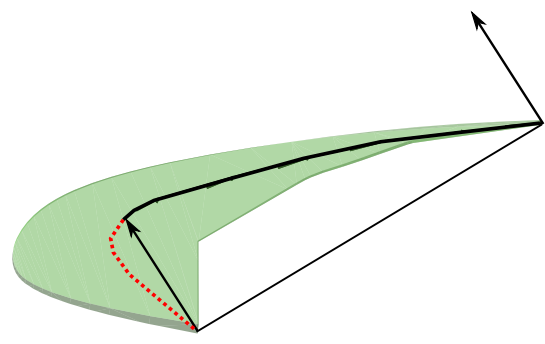

(a) Model A (not possible).

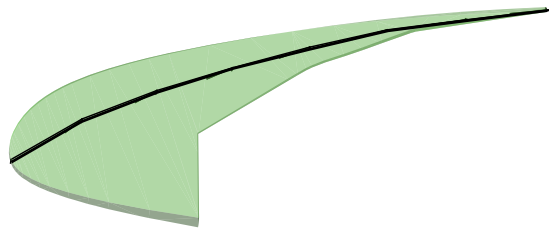

(b) Model B (selected).

Figure 3.6: NASA Trap Wing slat camber models.

Table 3.1: Analyzed NASA Trap Wing configurations from the LaRC 14x22ft Entry.

\begin{tabular}{c|cc|cc} 
& \multicolumn{3}{|c|}{ Slat } & \multicolumn{3}{c}{ Flap } \\
Config. & Deflection & Gap/Chord & Deflection & Gap/Chord \\
\hline 1 & 30 & 0.015 & 25 & 0.015 \\
8 & 30 & 0.015 & 20 & 0.015
\end{tabular}

For the study presented below, results were obtained using relaxed and fixed wake models. When using a fixed wake model, the wake is aligned with the freestream direction as it is emitted from the trailing edge of each lifting surface. In contrast, using a relaxed wake allows the wake elements to move with the local velocity field.

The figures below provide a graphical representation of the predicted relaxed wake shape of the NASA Trap Wing LaRC Config. 1 after 20 timesteps, at an angle of attack of 15 degrees. Note that the slat, main element and flap (plotted in grey) each shed their own vortex sheets into the wake from their respective trailing edges. Plotting each of these wakes in different colors provides a relatively simple method to distinguish between elements. Figure 3.7 shows an isometric view of the system, with the slat, main wing and flap moving towards the bottom left of the figure. The plot shows the wake shed from the slat (plotted in dark blue) passing through the main wing at multiple locations. Although this is physically impossible, it is of little concern since the slat itself has very little circulation and thus little effect on the overall forces. In addition, Willis shows that the velocities induced by a body piercing wake are almost identical to that of a properly treated body conforming wake [40]. 


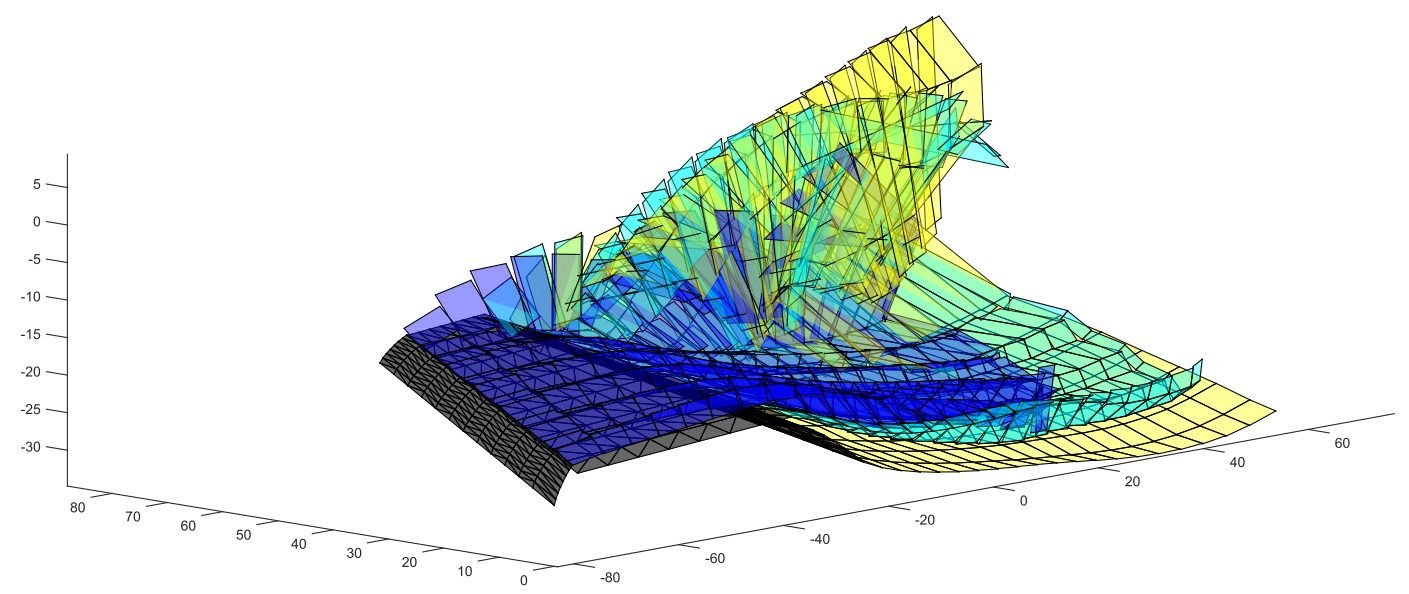

Figure 3.7: NASA Trap Wing LaRC Config. 1 wake plot after 20 timesteps at an angle of attack of 15 degrees.

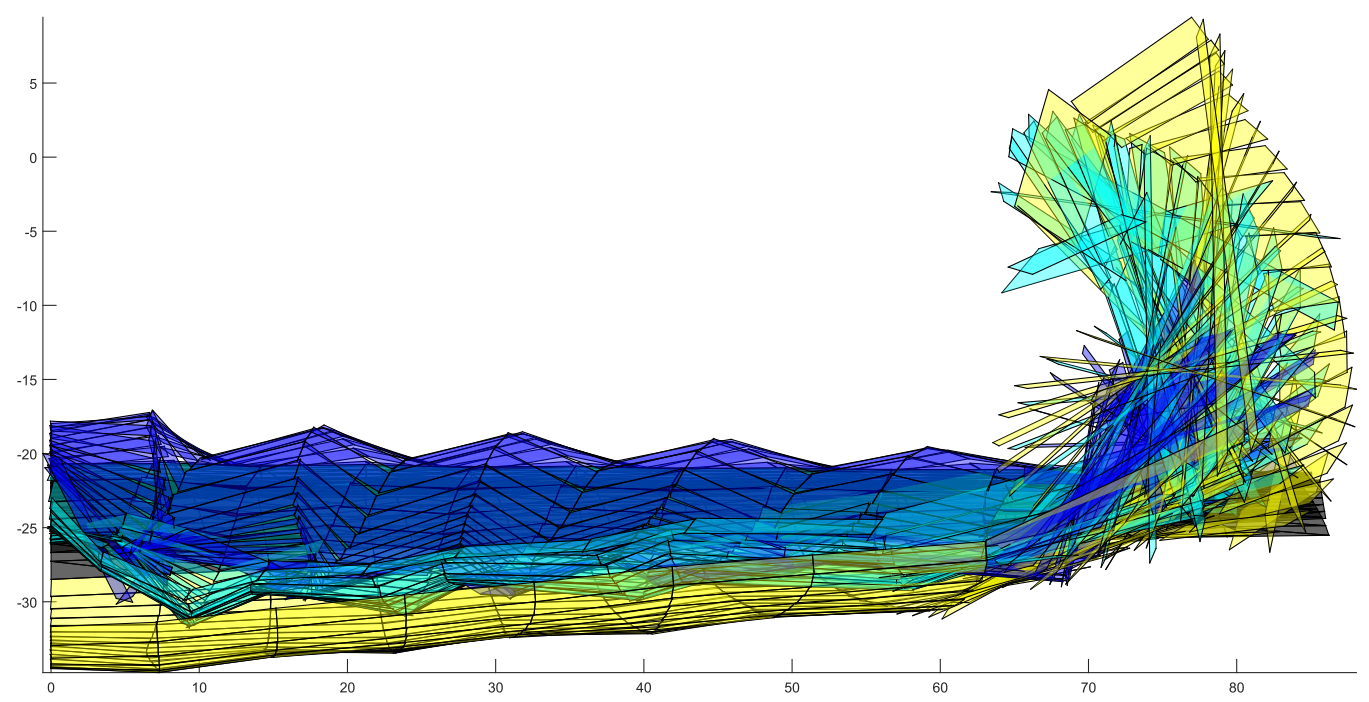

Figure 3.8: NASA Trap Wing LaRC Config. 1 wake plot after 20 timesteps at an angle of attack of 15 degrees, as viewed from downstream looking towards the trailing edge.

Figure 3.8 shows the wake plotted from downstream of the three element system. Clearly visible is the roll up of the right edge of the wake. Note that both Figs. 3.7 and 3.8 show the wake passing through itself. As mentioned previously, the robustness of this method allows this to happen without numerical issues occurring. In addition, the bumpiness of the wake in the spanwise direction, as seen 
clearly by the vortex sheet shed by the slat in Fig. 3.8, does little to affect the results, again, since the lightly-loaded slat has little effect on the overall forces. This effect is probably due to the local induced velocities as the wakes interact with each other and the lifting surfaces.

The time stepping method used results in large changes in the strength of the shed vorticity during the first few timesteps. Figure 3.9 shows the convergence of the span efficiency factor for the NASA Trap Wing LaRC Config. 1 as the wake evolves, using both relaxed and fixed wake models. In this case, the slat, main wing and flap are each modeled using 10 spanwise elements and 12 chordwise elements each, for a total of 360 surface elements. Convergence can be achieved for both fixed and relaxed wake models after approximately 20 timesteps; the only difference is that the results obtained using a relaxed wake have slight variations that quickly dampen. For all of the analyses presented, the time stepping distance has been set to one quarter of the main wing's root chord length. Therefore, the effects of the time stepping distance are not being studied here. Note that these results, and all results presented from this point on, have been found using triangular distributed vorticity elements, as shown in Fig 3.4.

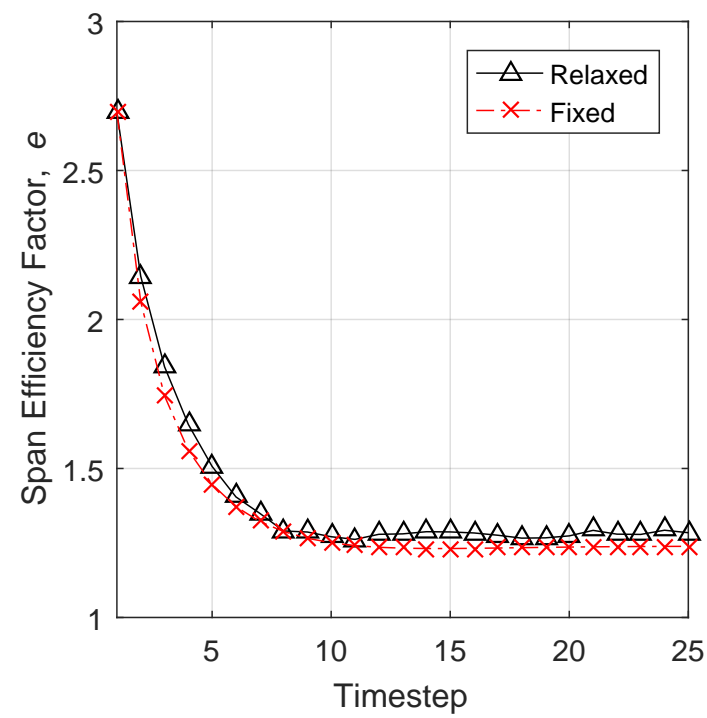

Figure 3.9: Convergence of the predicted span efficiency factor of the NASA Trap Wing LaRC Config. 1 as the number of timesteps is increased.

The results were obtained using a personal computer with an Intel ${ }^{\circledR}$ Core $^{\mathrm{TM}}$ i7 processor capable of running at $2.9 \mathrm{GHz}$. The computation time required to attain these results is plotted in Fig. 3.10. Since the number of elements in the wake grows which each timestep, the computation time per iteration 
increases as the analysis continues. In addition, Fig. 3.10 clearly shows a required increase in convergence time for a relaxed wake model, due to the relaxation process. On average, an analysis using a relaxed wake model required $25 \%$ more time than fixed wake analysis.

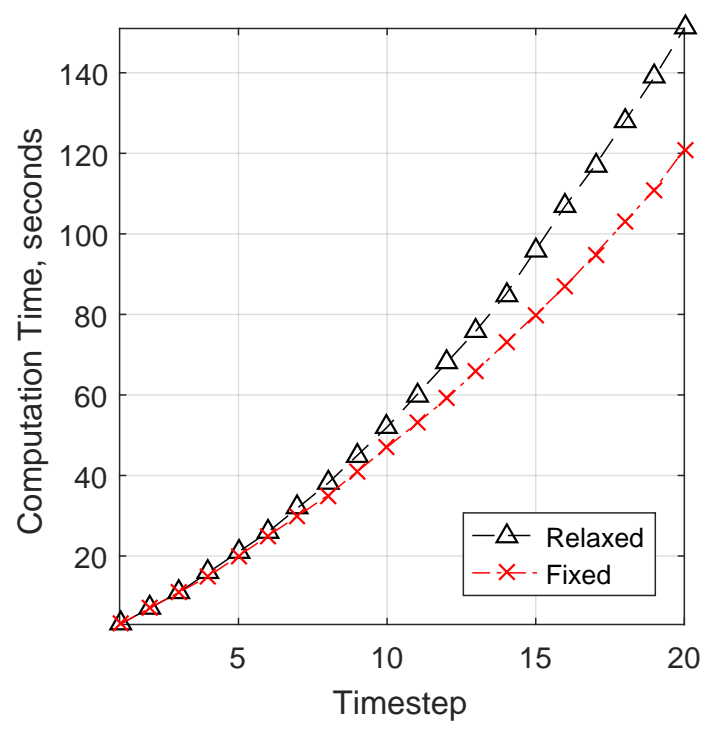

Figure 3.10: Computation time required for a single angle of attack analysis of the NASA Trap Wing LaRC Config. 1 as the number of timesteps is increased. Results were found on a personal computer using an Intel Core i7 processor capable of running at $2.9 \mathrm{GHz}$.

Figures $3.11 \mathrm{a}$ and $3.11 \mathrm{~b}$ present the convergence of the span efficiency factor as the number of spanwise elements, $n$, and chordwise elements, $m$, are increased. Convergence is found when 10 spanwise and 12 chordwise elements are used. Since this method is designed to be used in an iterative conceptual design process, when many analyses need to be run quickly, the least number of elements should be used that still generates a reliable solution. Figure 3.12 shows the average computation time for a single angle of attack analysis for the NASA Trap Wing, as the number of elements used to model the surfaces is increased. These results were achieved with 20 timesteps, the least amount possible while still achieving convergence, as seen in Fig. 3.9. In comparison to the results presented in Table 2.1, the results achieved here were computed with significantly lesser computational expense.

In addition to using the least number of elements to achieve convergence in the least amount of time, Fig. 3.12 shows that a fixed wake analysis requires less computational effort than a relaxed wake analysis. This is expected, since the relaxing procedure must be run each timestep, and the number of 


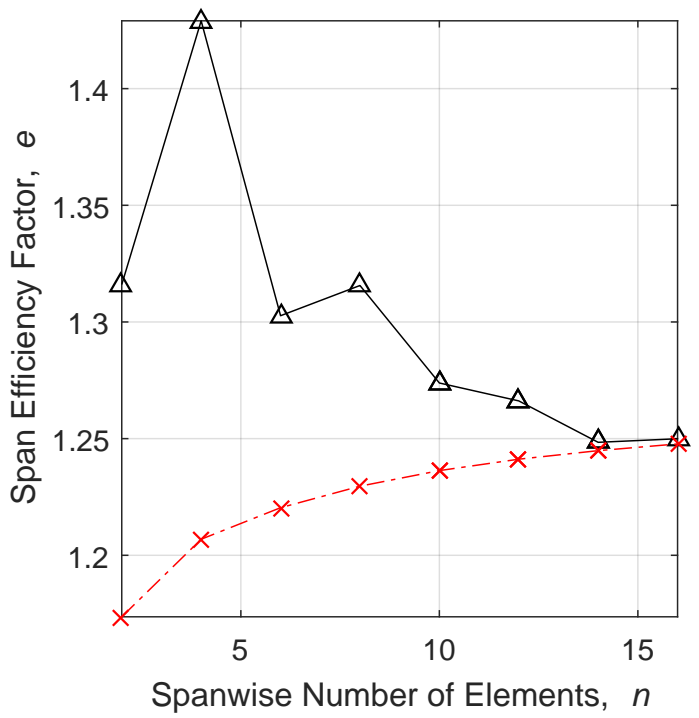

(a) Varying number of spanwise elements, $m=12$.

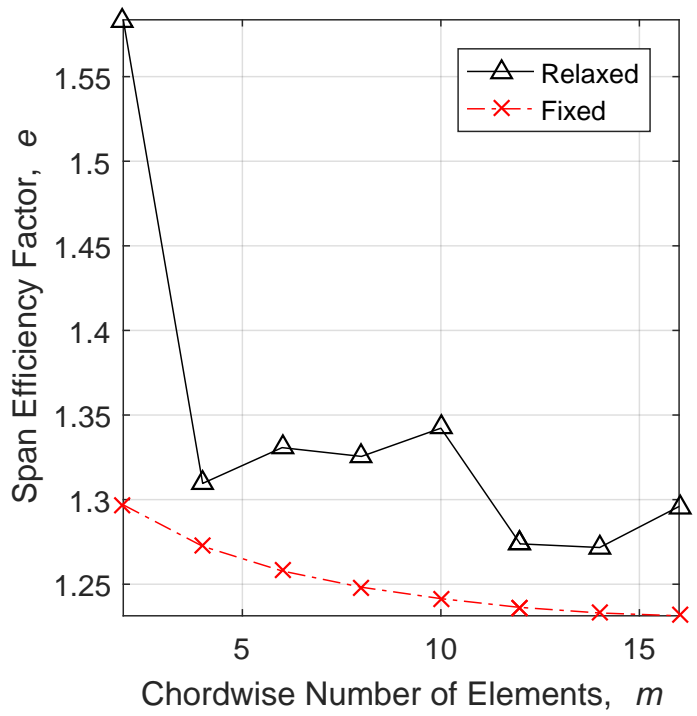

(b) Varying number of chordwise elements, $n=10$.

Figure 3.11: Convergence of the span efficiency factor as the number of elements is increased for the NASA Trap Wing LaRC Config. 1 at an angle of attack of 10 degrees.

wake elements increases as the number of spanwise elements on the lifting surfaces increase.

Knowing the differences in the aerodynamic predictions due to the wake type used is important, since a fixed wake can be used to save time over a relaxed wake. Analyzing both Figs. 3.11 and 3.12 , it can be seen that a fixed wake approach saves computational effort, with little deviations in the results as compared to a relaxed wake approach. In practice, these differences are not always known. For this reason, a study on the effects of wake shapes on aerodynamic predictions was completed and compiled in Ref. 41, and is summarized in Chapter 5.

Figures 3.13 and 3.14 show the predicted lift and drag values for the two NASA Trap Wing configurations that were analyzed. It is assumed that above an angle of attack of 30 degrees and below 5 degrees, viscous effects become large. Since this method neglects viscous effects, this study only compares the linear range inbetween 5 and 30 degrees of angle of attack. For both configurations, lift predictions show good agreement with the wind-tunnel data, for both wake shapes. This suggests that this method can be used to quickly predict changes to the lift coefficient due to small flap deflections with little error.

A limitation of this method can be seen in the drag values presented in Figs. 3.13a and 3.14a. Since profile drag is not predicted, there is a large difference between the predicted drag values and the wind- 


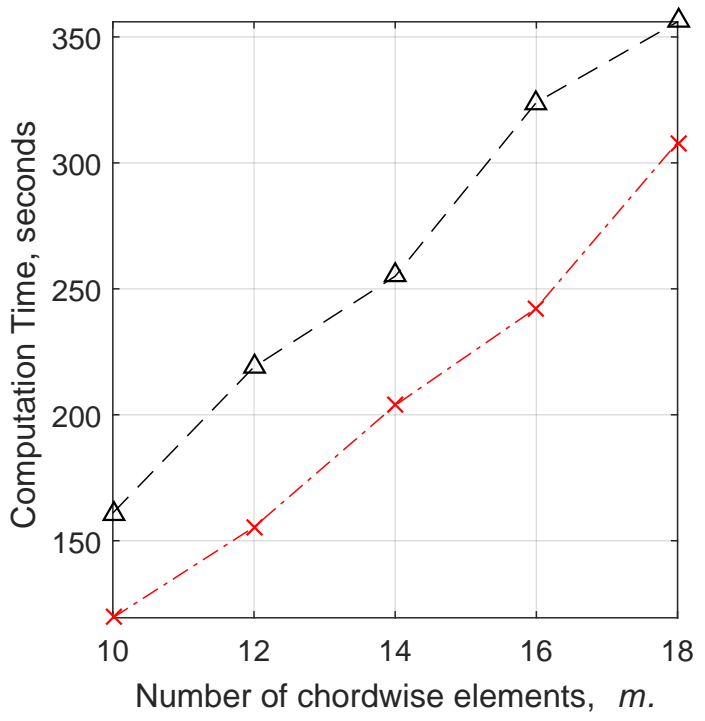

(a) Various number of chordwise elements, with 12 (b) spanwise elements.

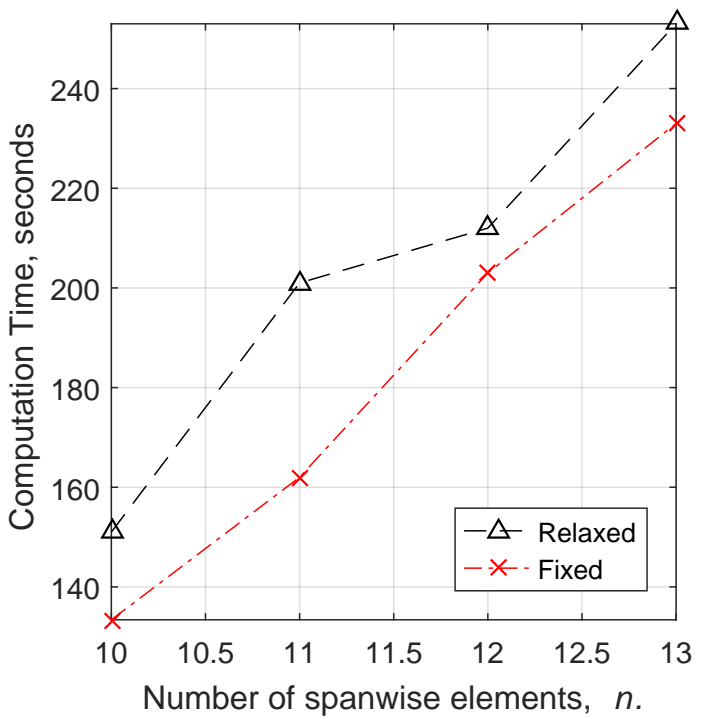

(b) Various number of spanwise elements, with 14 chordwise elements.

Figure 3.12: Effect of the number of elements on the computation time for a single angle of attack analysis using an Intel Core i7 processor capable of running at $2.9 \mathrm{GHz}$.

tunnel data. To estimate profile drag, the following method is used, as shown in Fig. 3.15. For a given lift coefficient, the predicted induced drag is subtracted from the NASA Trap Wing total drag that was measured experimentally, thus leaving only the profile drag forces. This profile drag prediction can then be added back into induced drag predictions for other configurations to estimate total drag. Of course, this method requires the total drag of the system before hand, and can only be used when analyzing small performance deltas due to configuration changes. Nevertheless, it is a relatively simple and fast approach to estimate the entire performance of a complex geometry.

Overall, differences appear in the induced drag predictions for a given lift coefficient, especially at high angles of attack. These differences are due to the changes in wake induced velocities along the trailing edge locations with the different wake types, and are explained in the wake shape study in Chapter 5. 


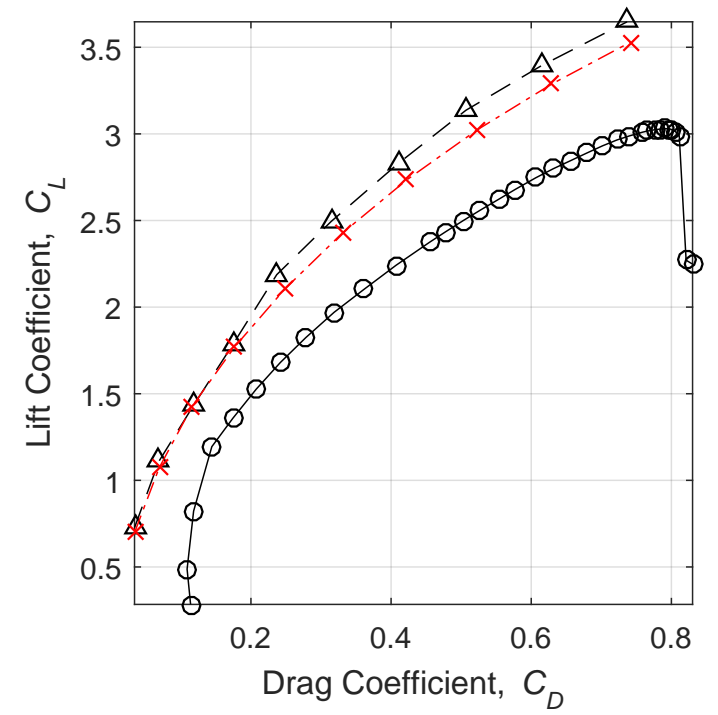

(a) Drag Polar.

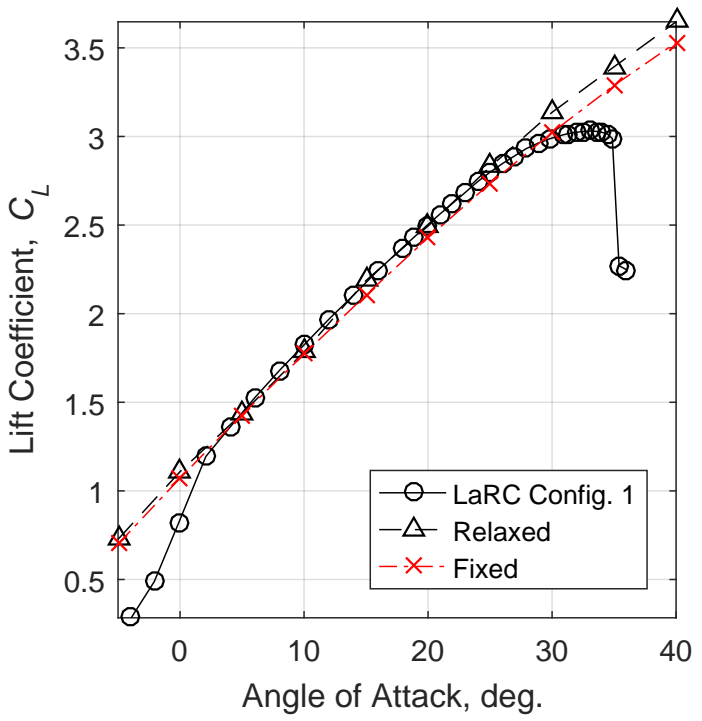

(b) Lift Curve.

Figure 3.13: Aerodynamic predictions of the NASA Trap Wing LaRC Config 1. Flap deflected 25 degrees.

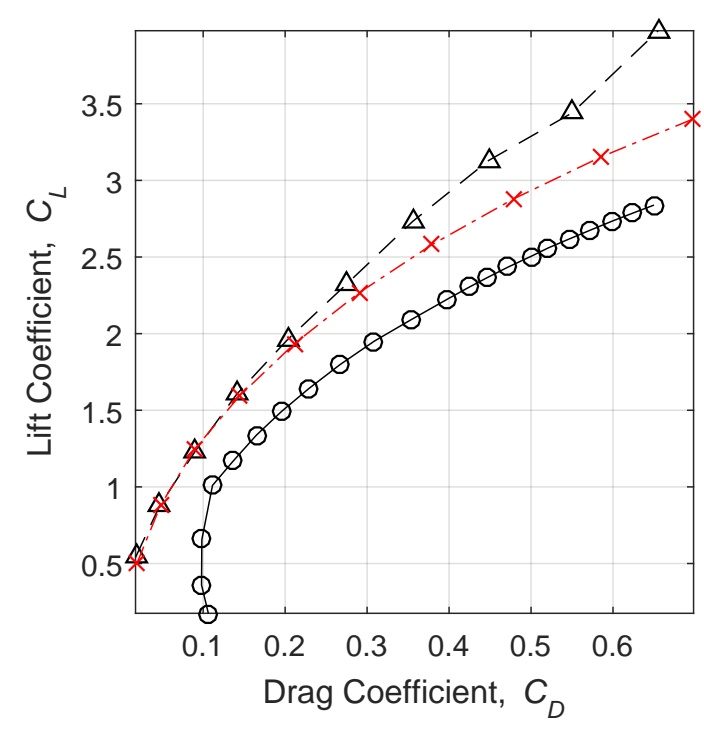

(a) Drag Polar.

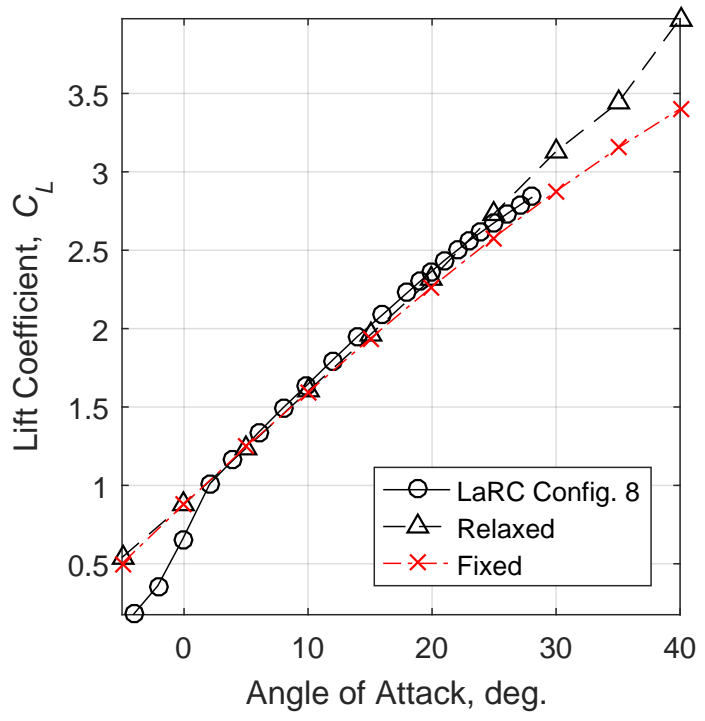

(b) Lift Curve.

Figure 3.14: Aerodynamic predictions of the NASA Trap Wing LaRC Config. 8. Flap deflected 20 degrees. 


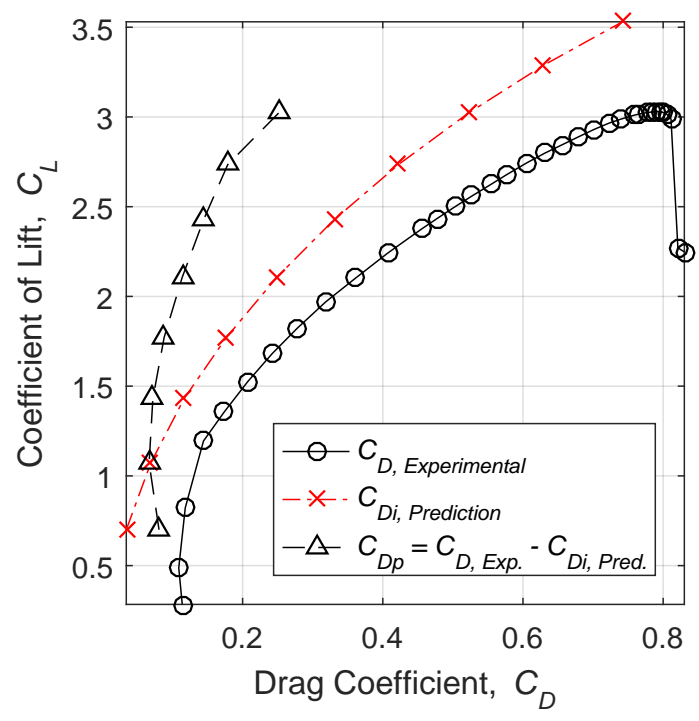

Figure 3.15: Total, induced, and profile drag of the NASA Trap Wing LaRC Config. 1.

\subsection{Summary}

Studies were undertaken with the developed investigative tool in order to demonstrate its suitability for an inviscid analysis of high-lift systems during the conceptual design phase. Both fixed and relaxed wake models were employed to compare the computed lift and induced drag coefficient predictions with experimental data. Although small differences were evident in the predictions of the two wake models, analysis of the results indicates that the tool is generally capable of computing lift and induced drag values. The predicted results show good agreement with the experimental data. Two configurations were tested, which showed that changes in lift due to a flap deflection are captured well.

It is also evident that the fixed wake model is computationally less expensive than the relaxed wake model. Lift predictions are similar when comparing the two wake types, however induced drag predictions vary, especially at high lift coefficients. Determining the reasons for these differences were the primary motivation behind the wake shape study presented in Ref. 41, and summarized in Chapter 5.

Overall, this method is proposed as a quick analysis tool for high-lift devices during the conceptual design phase that requires an iterative approach. Its relatively low computational needs make possible that a single analysis can be completed in minutes using a personal computer, compared to hours required by modern CFD solvers, even when using hundreds of computing nodes. 


\section{Chapter 4}

\section{Extension to a Panel Method}

The maximum lift of an aircraft is an important design parameter during the conceptual design stage, since it is critical for takeoff and landing calculations. Unfortunately, the prediction of $C_{L, \max }$ is difficult with most analysis methods, including advanced RANS solvers [2]. The limited ability of most analyses methods to predict maximum lift, coupled with the yet to be specified geometry during the conceptual design phase, are challenges for an accurate prediction. As a consequence, theoretical limits and comparisons to empirical data are often used to quickly estimate the maximum lift capabilities of a design.

There are multiple methods to predict maximum lift limits of a design, some based on theoretical calculations and some based on wind tunnel data. All of these methods, however, rely on a prediction of surface pressures, in particular of the leading edge suction peak. In order to find these pressures, the lifting surface method described previously was extended to a full panel code. A full panel code provides the ability to model thickness effects and attain surface pressure distributions.

\subsection{Prediction of Maximum Lift}

Several maximum lift conditions were outlined by Smith [7]. These methods are based on theoretical calculations of flow properties and observations made from wind tunnel testing. One method Smith described is the critical pressure coefficient that uses isentropic flow relations at a Mach number of 1 . Another method is J.P Mayer's 0.7 vaccuum relation, which finds a theoretical limit to the pressure 
coefficient assuming a surface static pressure of 0.7 of vacuum pressure over the upper-surface of the wing. Although these methods provide some insight into the theoretical maximum lift capabilities of an aircraft, they have some disadvantages, as discussed further below. Another approach to predict maximum lift is the pressure difference rule of Valarezo and Chin [17]. The pressure difference rule is largely based on maximum lift capabilities of airfoils in wind tunnels and adds the effect of Reynolds numbers to the prediction.

Smith describes that a theoretical maximum lift condition occurs when the highest local velocities over an airfoil reach a Mach number of 1.0. If flow at a point on the surface of a wing is considered to be isentropic, the isentropic flow relations can be formulated with the definition of dynamic pressure and speed of sound to solve for the the local pressure coefficient, $C_{p}$. This relationship can be given as a function of local Mach number, $M$, the freestream Mach number, $M_{\infty}$, and the ratio of specific heats, $\gamma_{s p}$, as:

$$
C_{p}=\frac{2}{\gamma_{s p} M_{\infty}^{2}}\left[\left(\frac{1+0.2 M_{\infty}^{2}}{1+0.2 M^{2}}\right)^{3.5}-1\right]
$$

The position where the local Mach number is equal to 1.0 will have the critical pressure coefficient, $C_{p}^{*}$, which depends only on the freestream Mach number. This simplifies Eq. 4.1 to:

$$
C_{p}^{*}=\frac{2}{\gamma_{s p} M_{\infty}^{2}}\left[\left(\frac{1+0.2 M_{\infty}^{2}}{1.2}\right)^{3.5}-1\right]
$$

This isentropic relation will give a minimum $C_{p}^{*}$ of -13 for a freestream Mach number of 0.223 , which is similar to minimum suction peak values obtained in wind tunnel testing for single element airfoils [17]. A limiting $C_{p}^{*}$ of -13 is a useful 'rule of thumb' for preliminary high-lift calculations [17, 42]. A major flaw, however, is that this rule is independent of Reynolds number, which has a strong influence on maximum lift. In general, an increase in Reynolds number leads to a higher maximum lift coefficient. Additionally, this criterion is not applicable to airfoils with leading edge devices, which, as repeatedly measured in experiments, have shown to have suction peaks much lower than -13 [7].

A second limit to maximum lift can be set given with the assumption that the theoretical minimum pressure over the upper-surface is a perfect vacuum. Beginning with the definition of $C_{p}$ rewritten using 
the speed of sound in a calorically perfect gas:

$$
C_{p}=\frac{p-p_{\infty}}{1 / 2 \rho_{\infty} V_{\infty}^{2}}=\frac{p-p_{\infty}}{\left(\gamma_{s p} / 2\right) p_{\infty} M_{\infty}^{2}}
$$

a limit can be found by setting the local static pressure, $p$, equal to zero, which yields a limit of $M_{\infty}^{2} C_{p}=$ -1.43. Experimental evidence of lifting surfaces above a Mach number of 0.4 has, however, shown that the minimum pressure coefficient usually occurs when the upper-surface pressure is approximately 0.7 of vacuum pressure, which corresponds to a limit of $M_{\infty}^{2} C_{p}=-1.0$ in Eq. 4.3. This is equivalent to the surface static pressure being $30 \%$ of the freestream static pressure. This criterion is known as J. P. Mayer's 0.7 vacuum pressure relation $[7,17]$ and can be used as a theoretical limit when high-lift devices are used.

The semi-empirical method of Valarezo and Chin can be used to provide a maximum lift prediction for freestream Mach numbers below 0.4 [17]. The pressure difference rule, as it is often referenced to, is a method that relates the maximum pressure difference over an airfoil's upper-surface to wind tunnel data to estimate maximum lift, as a function of chord Reynolds number and Mach number. This relation is plotted in Fig. 4.1.

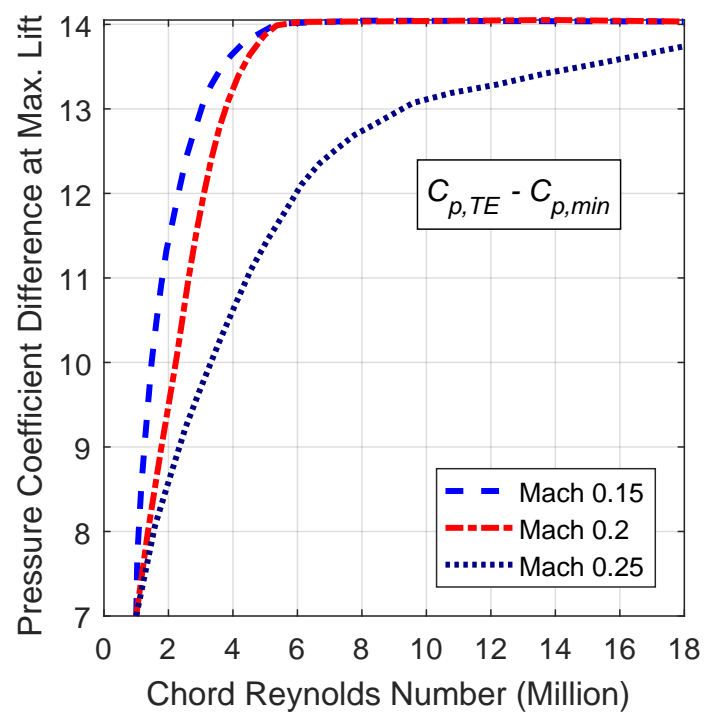

Figure 4.1: The pressure difference rule for maximum lift as a function of Mach number and Reynolds number. Adapted from Ref. 17. 
After producing surface pressure predictions, the method is performed on spanwise sections by taking the difference between the pressure coefficient at the trailing edge and at the suction peak. For the corresponding chord Reynolds number and flow Mach number, maximum lift is estimated when the magnitude of the value is equal to that found in Fig. 4.1. As a result, this method also predicts the critical location of where flow separation will first occur.

For a multi-element system, the pressure difference rule is applied on each of the elements. A properly configured multi-element system will, however, always stall when either the leading edge device or main wing stalls. This stall behavior is because the trailing edge devices are usually well protected from flow separation since, as the geometric angle of attack increases, the actual flap angle of attack decreases due to increasing downwash generated by the forward elements [17].

Valarezo and Chin show that wind-tunnel results of maximum lift of a wing with a high-lift device can be predicted well with the pressure difference rule. Figure 4.2 shows the prediction of maximum lift for a swept wing model with a leading edge slat and a trailing edge slotted flap. Although the angle of attack at maximum lift is not predicted well, the method is able to predict the maximum lift coefficient well for three different flap deflection angles on the system. The lift predictions in this example were found with an inviscid model with reduced flap deflections to account for viscous effects. This viscous decambering is a common practice in inviscid high-lift analyses to represent boundary layer losses [2].

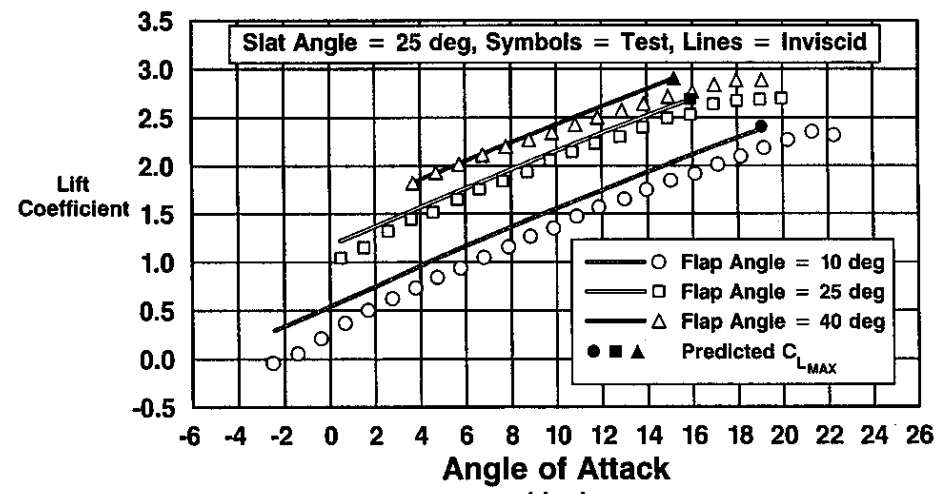

(deg)

Figure 4.2: Comparison of wind tunnel measurements to panel code predictions for the RAE wing with flaps and slats. Maximum lift is predicted using the pressure difference rule [17]. 


\subsection{Modifications}

To determine the applicability of these maximum lift prediction methods requires an analysis capable of finding the upper surface pressures. A lifting surface approach cannot be used for this due to the numerical infinite suction peak at the leading edge. In order to achieve the desired pressures, while still having the benefits of the higher-order method described in Chapter 3, the distributed vorticity element method was adapted to a full panel method that models thickness effects.

The major difference between the developed panel method and the zero-thickness lifting surface model described in Chapter 3 is the definition of the geometry. To simplify the required changes, the geometry is defined by 'wrapping' a lifting surface around the wing. As is shown in Fig. 4.3, the leading edge of the lifting surface becomes the lower-surface trailing edge in the panel code, and the lifting surface trailing edge becomes the trailing edge upper-surface. The current implementation of the lifting surface method easily allows for this transformation by taking care of the respective element orientations, normal directions and force directions.

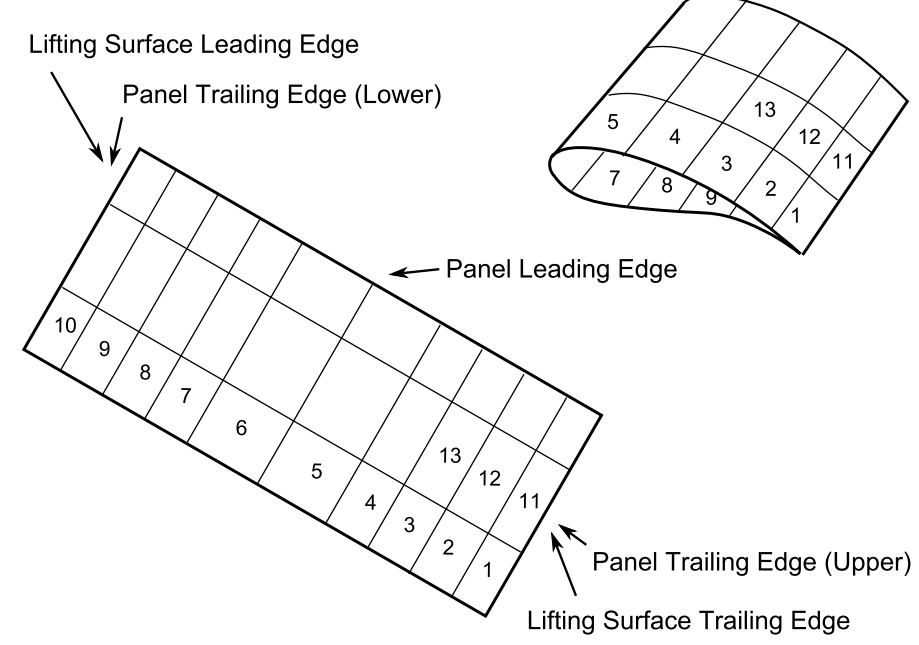

Figure 4.3: Method of defining panel geometry, beginning with the lifting surface method. Adapted from Ref. 12.

This modification required a few specific changes besides the modeling of the geometry. Specific attention is required at the trailing edge, since two lifting surfaces become joined at this location. To 
satisfy Kutta condition, the generation of the wake elements must now take into account the vorticity shed from the upper and the lower surfaces. As shown in Fig. 4.4, at the wing's trailing edge, the upper-surface element has a trailing edge strength of $-\Gamma_{U}$, the lower-surface trailing edge has a strength of $+\Gamma_{L}$, and the leading edge of the wake strength is defined as $+\Gamma_{W}$ [12]. If any circulation is present along the trailing edge, velocities normal to the freestream will be induced and flow will not leave the trailing edge smoothly. In order to satisfy Kutta condition, zero normal velocity and thus zero circulation must be present at the wing trailing edge,

$$
-\Gamma_{U}+\Gamma_{L}+\Gamma_{W}=0
$$

and therefore, the strength of the wake elements is the difference of the trailing edge upper and lower surfaces:

$$
\Gamma_{W}=\Gamma_{U}-\Gamma_{L}
$$

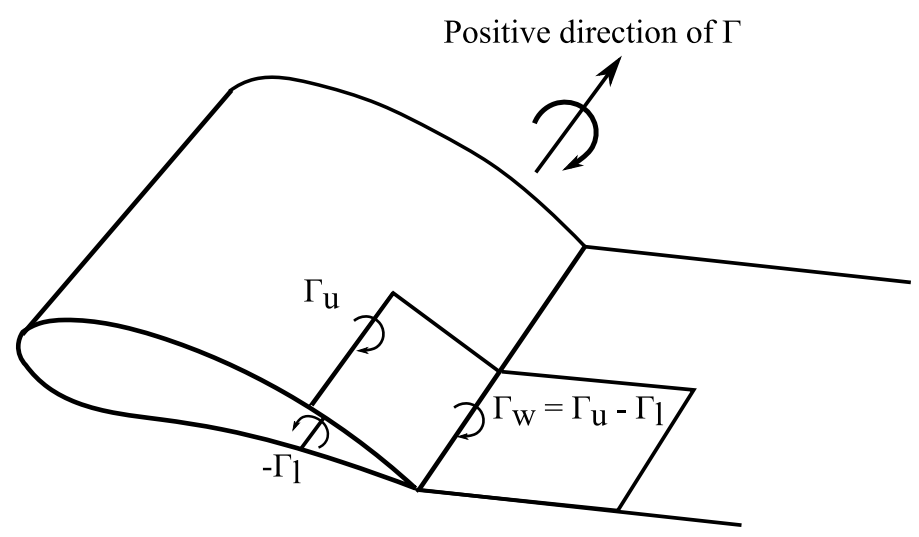

Figure 4.4: Implementation of the Kutta condition with a panel method. Adapted from Ref. 12.

Equation 4.5 extends to the vorticity in the wake as well. Since the vorticity in the wake, $\gamma_{w}$, is the differential of the spanwise circulation at the leading edge of the wake, $\Gamma_{w}$, the wake's vorticity can be found as the difference of the upper and lower surface vorticity.

After correctly defining the wake, the lift and induced drag forces require treatment since both involve 
the trailing edge of the lifting surfaces. The lift force requires attention since it is determined along the bound circulation, which, in the panel method, includes the trailing edge of the wing. This is because, as is seen with element number 10 in Fig. 4.3, the lifting surface leading edge becomes the lower-surface trailing edge in the panel method. In the lifting surface method, element number 10 is included in the lift force calculation since it has a defined circulation strength. However, in the panel method, element number 10 is moved to the trailing edge where there is zero overall circulation. To account for this in the numerical solution, the lower-surface trailing edge row of elements is removed from the lift force calculation. This is done for the surface normal force as well as the wake induced lift force.

Attention is also required to the numerical implementation of the induced drag, which is found by applying the Kutta-Joukowski theorem along the trailing edge using the same method as described in Chapter 3. In this method the drag force is calculated as the cross product of the wake induced velocity and the circulation at the wake leading edge, $\Gamma_{W}$. To implement this in the panel method, the circulation must be adjusted according to Eq. 4.5, in order to account for both the vorticity shed from both the upper and lower surfaces.

Since the panel method models thickness, a Dirichlet boundary condition can be used to solve for the singularity distributions required to set the total velocity inside the bodies to zero. However, a Dirichlet boundary condition is not used, since the method that is currently used to implement boundary conditions to the solution can only force a single component of the velocity to a desired value. To force all three velocity components inside a body to a specified value, as would be required with a Dirichlet boundary condition, would provide two extra boundary conditions. This would create an overconstrained system of equations, which would have to be solved using a 'curve fit' approach through each equation, and a final solution would require a method to reduce the associated errors. Because of the challenges associated with the Dirichlet boundary condition, the herein presented panel method uses a Neumann boundary condition that is identical to that used in the method of Chapter 3 , in order to satisfy flow tangency at control points on the panel surfaces.

\subsection{Surface Pressures}

Two methods were explored to determine the surface pressures, which are required for the prediction of maximum lift. The first method is based on the Kutta-Joukowski theorem, Eq. 2.1, which determines the 
local section normal force along the center line of each surface element using the local bound circulation, $\Gamma$, and the local chord length of each element, $c$. The subsequent section normal force coefficient is:

$$
c_{n}=\frac{N^{\prime}}{1 / 2 \rho V_{\infty}^{2} c}=\frac{\rho V \Gamma}{1 / 2 \rho V_{\infty}^{2} c}
$$

where the normal force, $N^{\prime}$, is a result of the Kutta-Joukowski theorem since the local velocity, $V$, is tangential to the surface and not in the freestream direction. This equation can be simplified to the form:

$$
c_{n}=\frac{2 V \Gamma}{V_{\infty}^{2} c}
$$

Since the surface pressure is of interest, the normal force coefficient can be related to the pressure coefficient knowing that the normal force coefficient is the difference between the upper and lower surface pressure coefficients:

$$
c_{n}=C_{p l}-C_{p u}
$$

The pressure term of actual interest is the pressure on the outside of the body, or the upper-surface pressure, $C_{p u}$, in this case. Theoretically, the velocity inside a body is zero, which leads to a stagnation pressure coefficient of unity under the surface. This results in $C_{p l}$ having a value of one, and the value of the desired pressure can be found as:

$$
C_{p u}=1-c_{n}
$$

Combining equations 4.7 and 4.9 , and dropping the $u$ subscript for simplicity, the surface pressure, as found from the section lift coefficients, is found as:

$$
C_{p}=1-\frac{2 V \Gamma}{V_{\infty}^{2} c}
$$

The second method of finding surface pressures uses surface velocities directly and relates them to the surface pressures according to Eq. 4.11, which non-dimensionalizes the surface static pressures to 
the freestream static pressure and is simplified with Bernoulli's equation to:

$$
C_{p}=1-\left(\frac{V}{V_{\infty}}\right)^{2}
$$

When applied to the developed panel code, the two methods of predicting surface pressures show vastly different results. Figure $4.5 \mathrm{a}$ shows the predicted surface pressures along a chordwise line at $52.5 \%$ of the span of a non-lifting cylinder of aspect ratio 20 . Immediately, it can be seen that the pressure predictions are not smooth. The waviness in the surface pressure plots are due to the use of triangular elements used to model the geometry. Since two triangles are required to make up one planar quadrilateral surface, only every second element is used to model geometry changes in the chordwise direction. As a result, every second element is required to have greater circulation strength to turn the flow.

When compared to the analytical solution for the pressures around a non-lifting cylinder, it can be seen that the sectional lift method of predicting surface pressures shows good agreement, while the direct surface velocity method under predicts the pressures. According to Eq. 4.11, this means that the surface velocities are too small. Figure $4.5 \mathrm{~b}$ shows the streamwise velocities, $u$, and the spanwise velocities, $v$, through the center of a cylinder as predicted with the current panel method and compared to the analytical streamwise velocities. Given that the freestream velocity is $1.0 \mathrm{~m} / \mathrm{s}$, the streamwise flow velocity at the top and bottom should be $2.0 \mathrm{~m} / \mathrm{s}$. Note how the velocities are correct just off the surface, however they are very small on the surface. This is likely due to the use of discrete vortex filaments to model the bound circulation. To resolve this, the vortex filaments should be replaced with vortex sheets to represent a continuous vorticity dirtribution in the chordwise direciton. Nonetheless, it can be reasoned that this is why the pressures as predicted with the direct velocity method are too small.

The surface pressures found using the local section lift method show good agreement to the analytical solutions for both lifting and non-lifting flows, and therefore, the sectional lift method of finding the pressures will be used herein. In addition, this method provides better insight into the accuracy of the method since the overall lift of the wing is found using the Kutta-Joukowski theorem and not by using a surface pressure integration approach.

Figure $4.5 \mathrm{~b}$ also shows that the velocities inside the body are not exactly zero (due to the use 


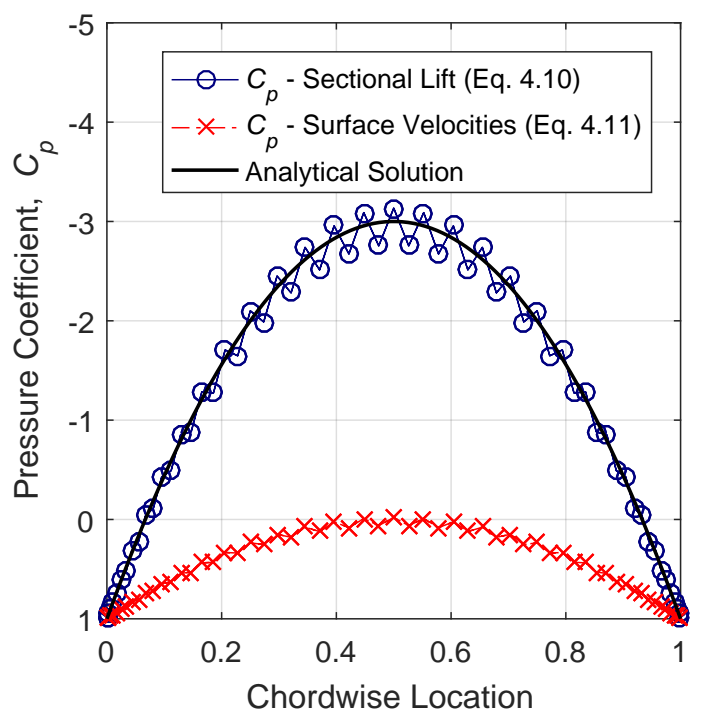

(a) Chordwise surface pressure predictions.

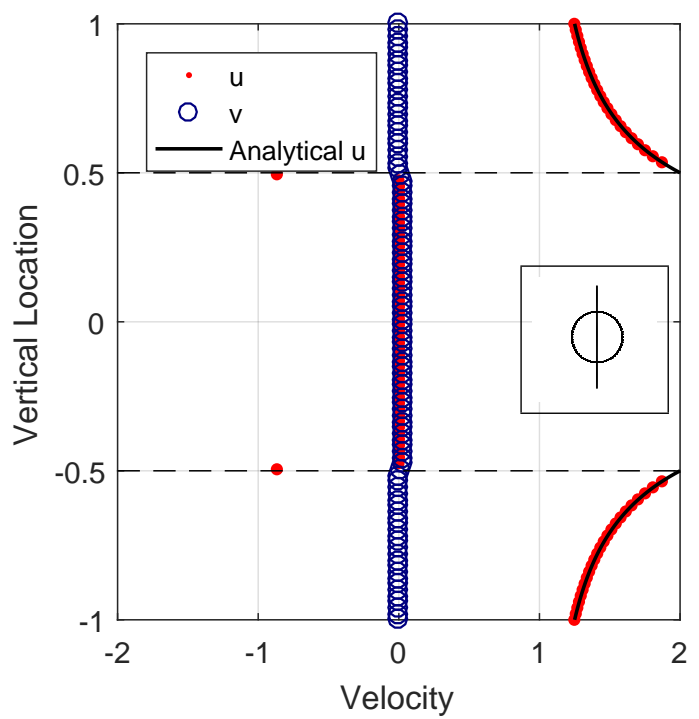

(b) Velocities along a vertical line through the center of a cylinder. The dotted lines represent the height of the top and bottom of the cylinder. The inset shows the velocity profile location on the cylinder cross section.

Figure 4.5: Analysis of pressures and velocities near the half span of a non-lifting cylinder of aspect ratio 20 exposed to a freestream flow of $1 \mathrm{~m} / \mathrm{s}$.

of a Neumann boundary condition and not a Dirichlet boundary condition). This should nullify the assumption made to generate Eq. 4.9 and also render the pressures found with the sectional lift method incorrect. However, as is shown in Fig. 4.5a, the surface pressures can be predicted well using the sectional lift method of Eq. 4.10. This effect is explored further in Fig. 4.6. When modeling a wing with a symmetric airfoil using 40 elements in the chordwise direction, spanwise flows exist inside the wing. On the surface, the velocities are equal to a value less than the velocity immediately above the surface, but greater than the velocity below the surface. The only known method to reduce the internal flow velocity is to increase the number of elements used to model the airfoil. As seen in Fig. 4.6b, increasing the number of chordwise elements to 120 sets the velocities inside the wing closer to zero. Nevertheless, small spanwise components remain and the velocities on the surface are incorrect.

The origin of these residual velocities can be described by looking at the idealized non-lifting system in Fig. 4.7. If the upper and lower surfaces of a non-lifting symmetrical wing that is at a zero angle of attack are treated as separate lifting bodies, each surface generates an equal lift force in opposing 


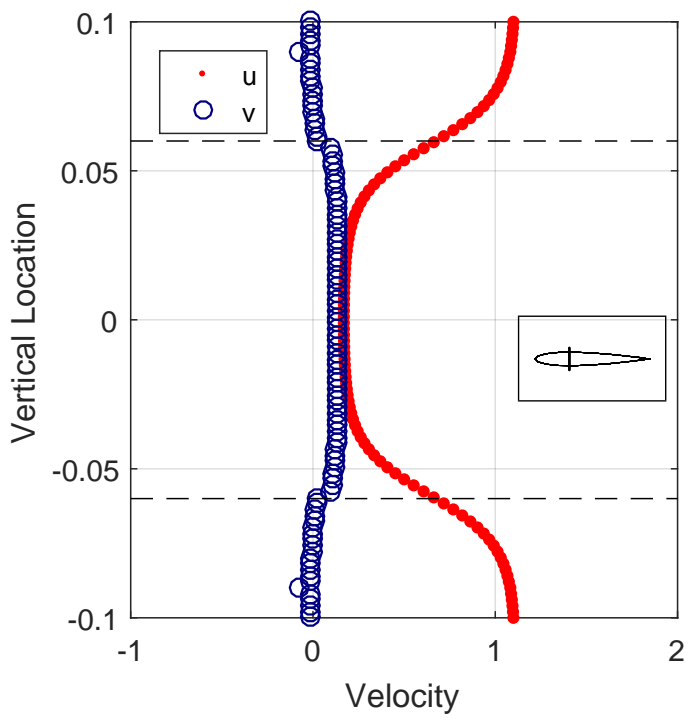

(a) 40 chordwise elements.

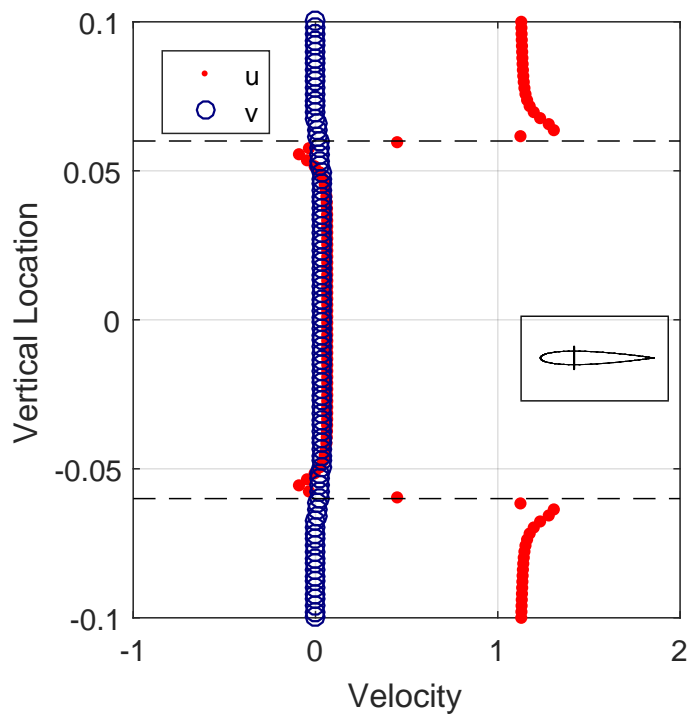

(b) 120 chordwise elements.

Figure 4.6: Velocities along a vertical line through the center of a non-lifting wing. The dotted lines represent the top and bottom of the wing. The inset figures show the locations of the velocity profiles on the airfoil cross sections.

directions. For a wing of finite span, this will result in vorticity distributions in the directions shown in Fig. 4.7, since it can be assumed that circulation will decrease towards the wingtips. This vorticity, contained in the vortex sheets, will induce inboard velocities above and below the wing, and outboard velocities inside the wing.

Inside the wing, the influence of the upper and lower surfaces are additive, causing a spanwise flow towards the wingtip within the bounds of the wing. Above the wing, the contribution of the uppersurface dominates over the contribution of the lower-surface, which is of the opposite direction but is reduced due to its relatively larger distance. This effect also applies to the lower-surface. As a result, for a symmetrical wing, the system is left with induced spanwise flow components that are relatively small outside of the wing, but relatively large inside the wing. Directly on the surface, the contribution of the local vortex sheet is ignored, and thus the induced velocities from the opposite surface are not countered with anything, leaving velocities on the surface which have outboard components. This effect becomes more pronounced as the upper and lower surfaces are moved closer together.

Reducing the internal spanwise flow components is one reason why a relatively large number of 


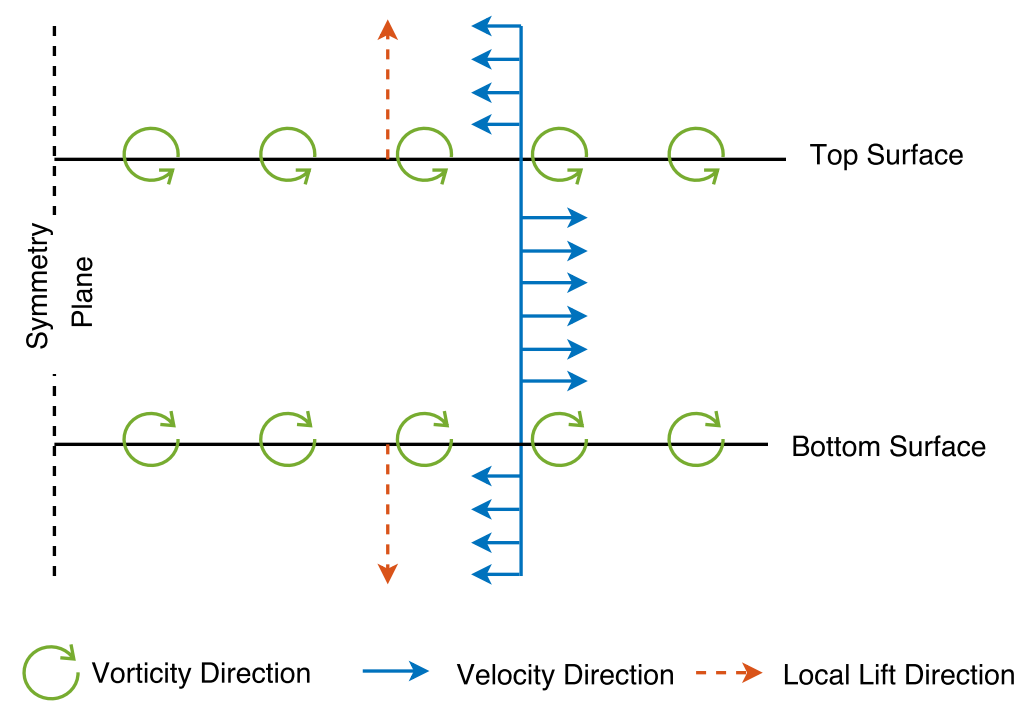

Figure 4.7: Predicted internal velocities due to the influence of the upper and lower surfaces.

elements is required to model surfaces using the herein presented method. A second reason is that surfaces with large curvature require fine discretization in order to properly define the geometry using planar elements. Unfortunately, the relatively large number of elements increases the computational time required for a converged solution.

When analyzing flows about a non-lifting body, computational effort is kept to a minimum because only one timestep is required since the wake is of zero strength. Figure 4.8 shows the comparison of the surface pressures that were derived using the herein described approach to inviscid XFOIL predictions. The surface pressures that were determined using the present approach were found at $52.5 \%$ of the span of a wing with a rectangular planform and aspect ratio of 20. A large number of elements were required both at the leading and trailing edges. Too many elements near the trailing edge, however, will cause a spike in the velocities, as can be seen when 120 elements are used to model the airfoil. This is due to the use of discrete vorticies that model the bound circulation, and depends on the trailing edge angle and number of elements located there.

For lifting bodies, a large number of timesteps are required, since the iterative solution method cannot satisfy Kutta condition and flow tangency in the same iteration. In addition, surface pressures are found at element leading edges, and not at the control points where flow tangency is established. As a result, the flow is not necessarily tangent to the surface at the locations where the pressures are found. This effect is especially noticeable near the trailing edge and increases with more elements positioned there. 


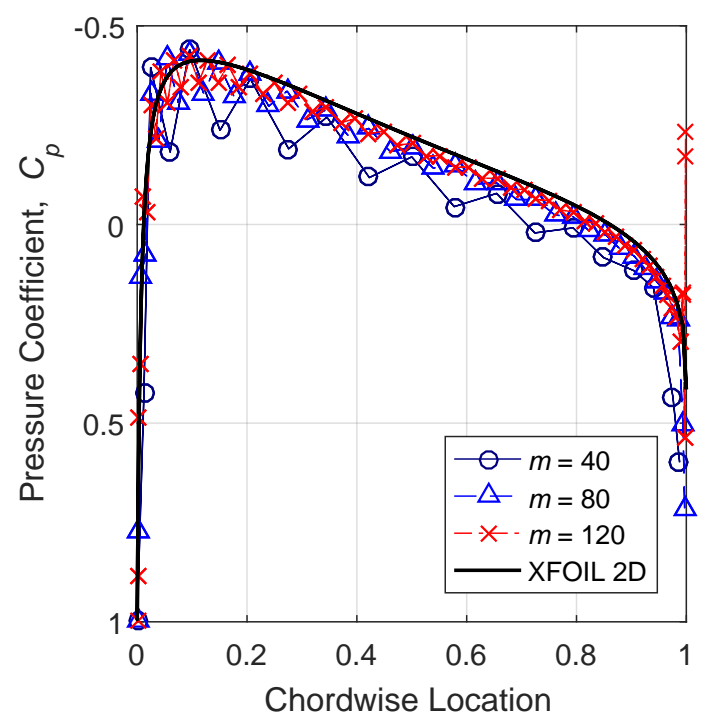

Figure 4.8: Convergence of surface pressure coefficients near the half span of a non-lifting rectangular wing with an aspect ratio of 20 , as the number of chordwise elements, $m$, is increased from 40 to 120.

The solution to this problem is to run more timesteps and allow the trailing edge upper and lower surface pressures to join. This, of course, means an increase in computational time needed to find a solution. Figure 4.9 shows this convergence of the pressure coefficient predictions at $52.5 \%$ of the halfspan of a

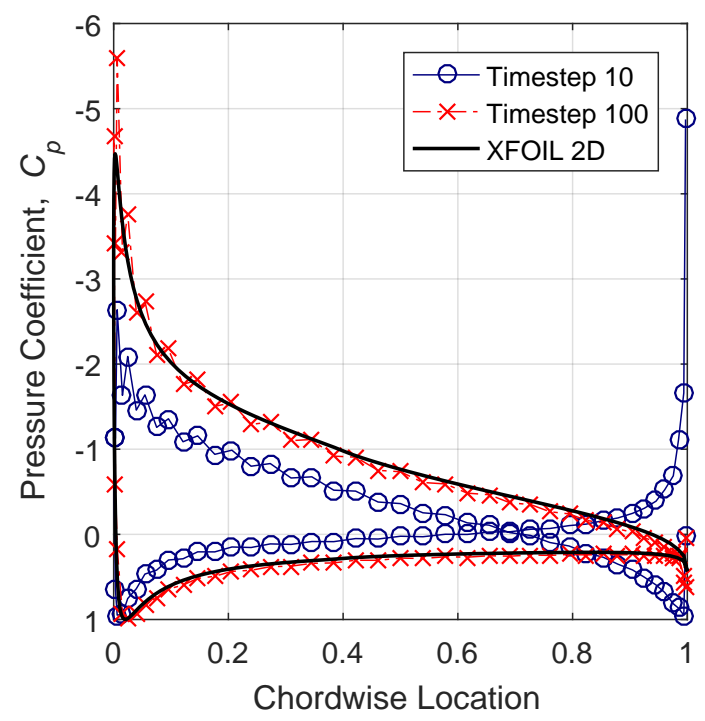

Figure 4.9: Convergence of surface pressure coefficients near the half span of a lifting rectangular wing with an aspect ratio of 20, as the number of timesteps is increased from 10 to 100. 
rectangular wing with an NACA 2412 airfoil, with an aspect ratio of 20, at an angle of attack of 10 degrees. The predictions are compared to the two-dimensional inviscid solution from XFOIL at the same section lift coefficient of 1.3. The leading edge pressure peak can be refined by adding more elements there, or by averaging the pressure coefficients to remove the waviness.

Allowing sufficient timesteps for a fully converged solution, the aforementioned maximum lift prediction methods can be applied to the upper-surface pressures of a wing section to estimate when the section stalls. An example application is shown in Fig. 4.10 that shows results when applying the pressure difference rule to spanwsise sections along the halfspan of a rectangular wing with an aspect ratio of 20. The plot shows the difference between the leading edge suction peak and the trailing edge pressure, and the corresponding maximum difference from the pressure difference rule, using Fig. 4.1, based on a chord Reynolds number of 2.3 million at a Mach number of 0.2 . The pressure difference rule method predicts that the wing first sees maximum lift between an angle of attack of 15 and 16 degrees. The method also suggests that the critical location where separation occurs first is the inboard section close to the wing root.

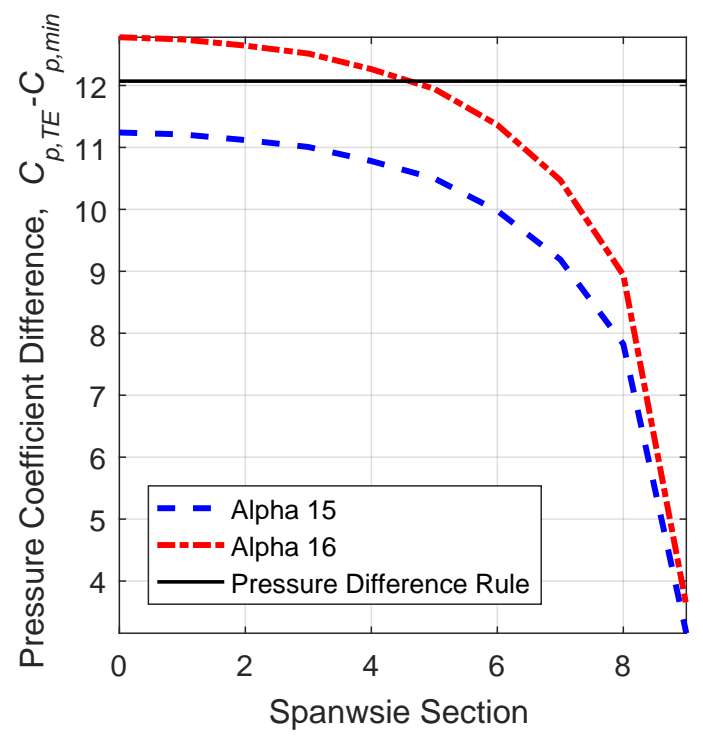

Figure 4.10: Maximum lift angle of attack as predicted by the pressure difference rule.

According to the isentropic relation, Eq. 4.2, the local Mach number of the flow reaches 1.0 when the minimum pressure coefficient is -16.3. This occurs when the angle of attack of the system is between 18 and 19 degrees. Finally, J.P Mayer's 0.7 vacuum pressure relation, which is based on high-subsonic 
Mach numbers, predicts maximum lift at a minimum pressure coefficient of -25 . This occurs at an even higher angle of attack.

\subsection{Example Application}

An analysis of the NASA Trap Wing Config. 1 was completed with the panel code to test the applicability of the maximum lift prediction methods to high-lift devices using the developed panel method. For this analysis, the Trap Wing was modeled using ten elements in the spanwise direction and forty elements to model the airfoil of each lifting surface, as shown in Fig. 4.11. This totals to 1200 elements. Referring to Fig. 3.11a, using ten elements to model the span shows good convergence in a relatively limited amount of time with the lifting surface method. Thus, it was used for the analysis with the panel method. Unfortunately, the number of chordwise elements has to be high in the panel method in order to accurately model the airfoil shapes, and the number of timesteps has to be large to achieve convergence.

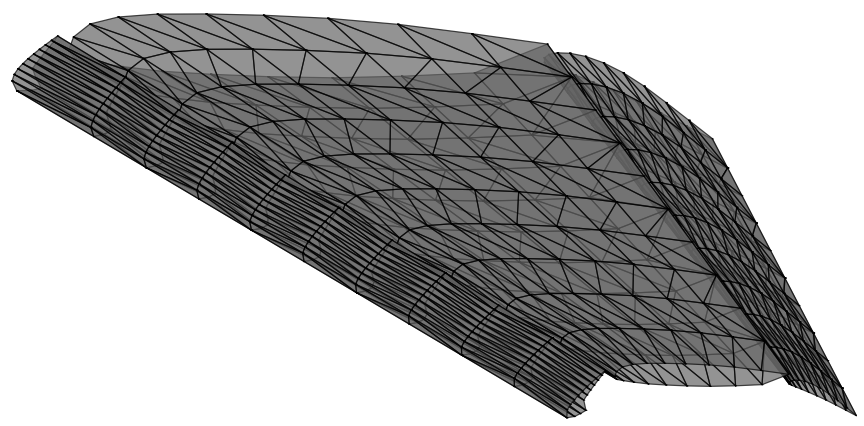

Figure 4.11: The NASA Trap Wing LaRC Config. 1 surfaces modeled using triangular elements.

Using 40 elements to model each of the NASA Trap Wing airfoil geometries represents the surfaces well, with two noticeable exceptions. These are shown in Fig. 4.12, where the CAD model represents the actual location of the surfaces. The first exception is the cove area at the trailing edge of the main wing, as shown in Fig. 4.12b. Dispersing the elements in the chordwise direction in a full-sinusoidal manner would increase the accuracy of the cove, however, for this analysis, the elements were positioned using a half-sinusoidal distribution to increase the number of elements at the leading edge. The second 
inconsistency in the model is the increase in the trailing edge angle of the slat, as shown in Fig. 4.12a. The tight geometry at this location can result in unwanted pressure peaks. To solve this, the angle between the upper and lower surfaces at the trailing edge of the slat has been increased from 0.8 degrees to roughly 25 degrees. There were no changes that were required to be made to the flap, as seen in Fig. $4.12 \mathrm{c}$.

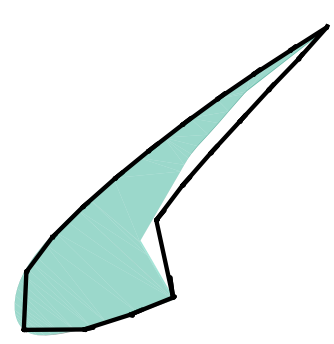

(a) Slat.

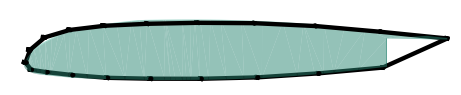

(b) Main Wing.

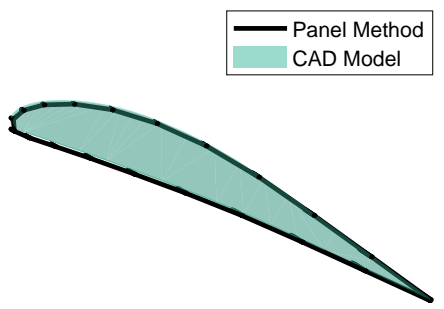

(c) Flap.

Figure 4.12: NASA Trap Wing Config. 1 airfoil approximations.

Figure 4.13 shows the prediction of lift and induced drag as found with the panel method using a fixed wake, and compared to the wind-tunnel results as well as the lifting surface predictions as presented previously in Chapter 3. As expected, there is an increase in the slope of the lift curve when compared to the lifting surface results, however, it is not as large an increase as expected. To reduce any errors caused by this, the flap angle should be reduced as it is commonly done with inviscid panel methods in order to compensate for decambering effects as the upper-surface boundary layer thickens [2, 17]. However, the flap angle was not corrected for the herein presented results.

In general, induced drag predictions of the panel method are larger than the predictions of the lifting surface method for given lift coefficients. Unfortunately, the exact reason for this is unknown. Comparing spanwise pressure distributions has suggested that a possible reason for these inconsistencies is that the predicted lift values are too low when using the panel method. If the lift predictions increased, the drag polar of the panel method would be shifted vertically upwards, closer to the prediction of the lifting surface. However, this is only a speculation into the inconsistencies, therefore no attempt was made to artificially 'correct' these results by offsetting the values, and the study was completed with the results shown in Fig. 4.13. 


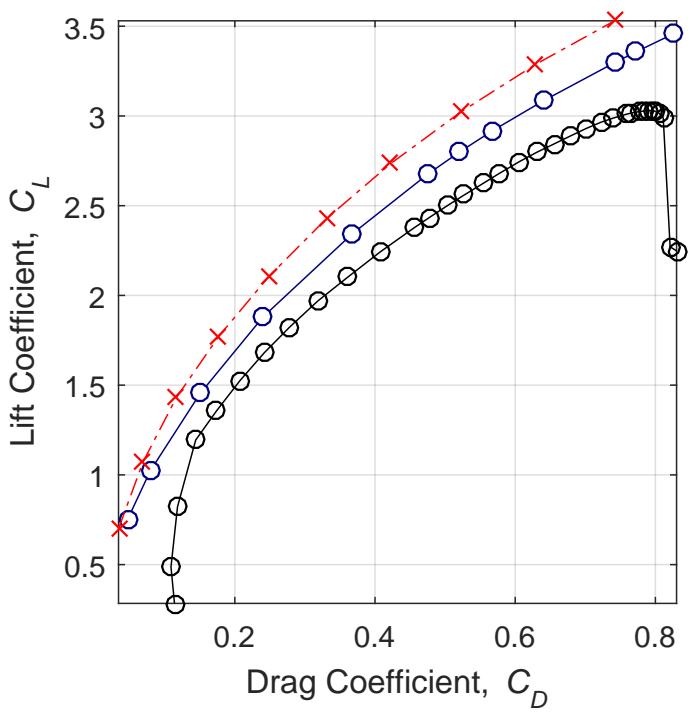

(a) Drag Polar.

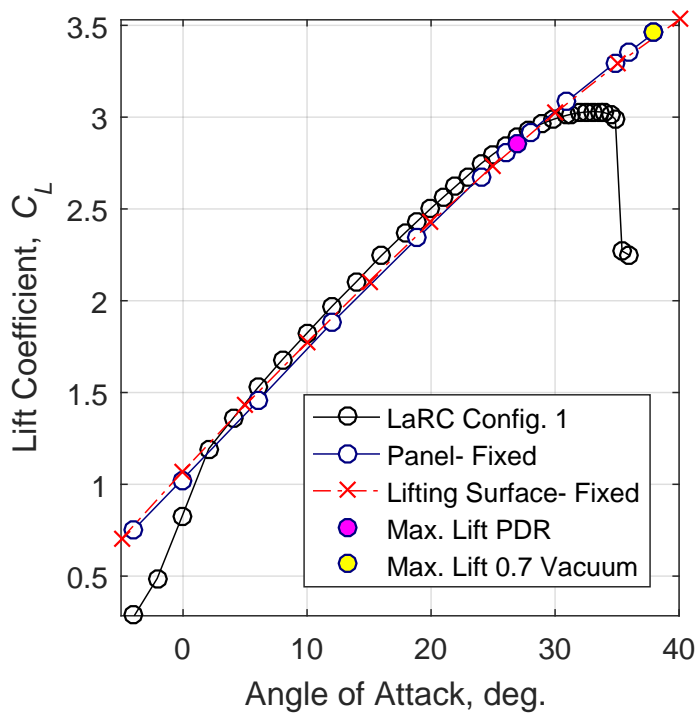

(b) Lift Curve.

Figure 4.13: Aerodynamic predictions of the NASA Trap Wing LaRC Config. 1 using a panel method with a fixed wake.

The maximum lift predictions of the pressure difference rule (PDR) and 0.7 vacuum relation are shown in Fig. 4.13b. The pressure difference rule predicts the maximum lift coefficient to within a $C_{L}$ of 0.18 , and the 0.7 vacuum relation finds the maximum angle of attack to within 3 degrees. These predictions are not as accurate as those made by RANS solvers in the AIAA CFD High-Lift Prediction Workshop (HiLiftPW-1), however, they were found with much less computational effort. These results required, on average, 21 minutes per angle of attack to achieve convergence on a personal computer using an Intel Core i7 processor capable of running at $2.9 \mathrm{GHz}$. Additionally, the accuracy of these maximum lift predictions could be increased with further studies into panel density effects.

As mentioned, the results of the panel method predictions presented above were found using a freestream fixed wake. Although a relaxed wake, free of singularity issues, can be modeled using this method, a problem occurs when modeling multiple closely interacting lifting elements. Errors are introduced when wakes are allowed to move inside a body where velocities are very small. When inside, this nonphysical effect causes the wakes to become 'stuck' in the body, as if they have become fixed to the body. A simplified example is shown in Fig. 4.14, where the relaxed wakes from a leading edge slat and a symmetrical main wing at an angle of attack of zero are plotted. In the figure, the halfspan wings are 
traveling from right to left with a symmetry plane at the location of the fuselage. As seen in the figure, the wingtip is modeled as an open end. Clearly visible is the wake emitted from the leading edge slat (plotted in dark blue) extending downstream into the main wing. Inside the main wing, the induced velocities are equivalent to the freesteam velocity, but in the opposite direction. This moves the wake from the slat forward the same distance that the main wing wing moves forwards in a given timestep. As a result, the wake emitted from the slat moves along with the main wing instead of extending downstream. At the open wing tip, a small outboard component of the velocity exists inside the main wing, causing the wake from the slat to leak out.

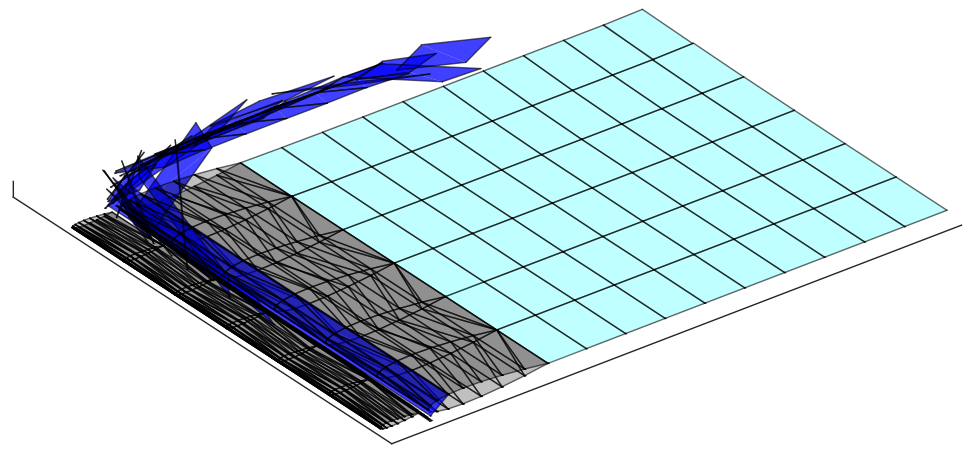

Figure 4.14: Simplified multiple lifting element system modeled with a panel code. This figure shows the wake from the slat becoming fixed inside the main wing.

When extended to more complex high-lift systems, the issue of a wake becoming fixed inside a body is unpredictable and occurs only at certain spanwise locations and at specific angles of attack. The unmodified relaxed wake shed by the slat of the NASA Trap Wing is shown in Fig. 4.15a. Figure 4.16 shows the lift and drag predictions using the panel code with an unmodified relaxed wake, and compares them to the results using a relaxed wake with the lifting surface method of Chapter 3 and the available wind-tunnel data. As can be seen, this unmodified relaxed wake introduces errors to the lift and induced drag predictions. Small errors are introduced to the lift and large errors are introduced to the drag predictions when using a relaxed wake.

This problem was explored by Olson and Albertson [43]. They provide two methods to try to remove the issue of the wake becoming fixed inside downstream elements. The first method involves modifying 


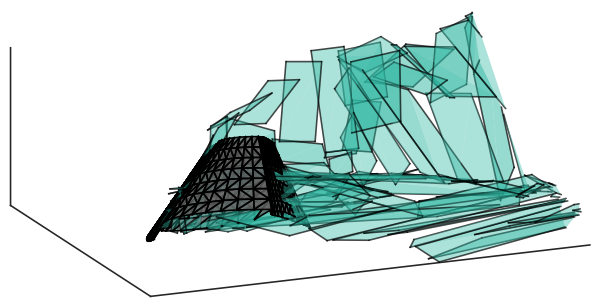

(a) An unmodified relaxed wake which moves with the (b) A modified relaxed wake case, in which the post local velocities.

Figure 4.15: The relaxed wake shed by the slat of the NASA Trap Wing modeled with a panel method at an angle of attack of 12 degrees.

the timestep size so that the wakes are correctly forced above or below downstream elements. This was attempted in this study, however it was deemed to be not a viable solution. Increasing the timestep size did not remove the issues entirely and decreasing the timestep size caused a large increase in the computation time necessary for convergence. The second method presented by Olson and Albertson is to remove the slat from the model, thus removing the issue of the slat wake passing through downstream elements. Since stall is assumed to be driven by the slat, it was felt that the method of removing the slat altogether was not useful without making further corrections.

In this study, the method used to reduce the errors with the relaxed wake was to force the post trailing edge row of elements emitted from the slat to an angle of 30 degrees above the horizontal, as seen in Fig 4.15b. The wake was then allowed to relax starting with the second downstream row. As a result, the location at which the velocity is computed in the relaxation procedure is always outside the wing, free of issues.

The lift and induced drag predictions that were found using the modified relaxed wake method are also shown in Fig. 4.16. The results behave much nicer across all the angles of attack tested, when compared to the unmodified relaxed wake. The lift predictions using the modified relaxed wake show good agreement to both the available wind-tunnel data and the relaxed wake predictions using the lifting surface method of Chapter 3. When compared to the lifting surface method, a large increase in the induced drag coefficent can be seen when using the modified relaxed wake. This is similar to 


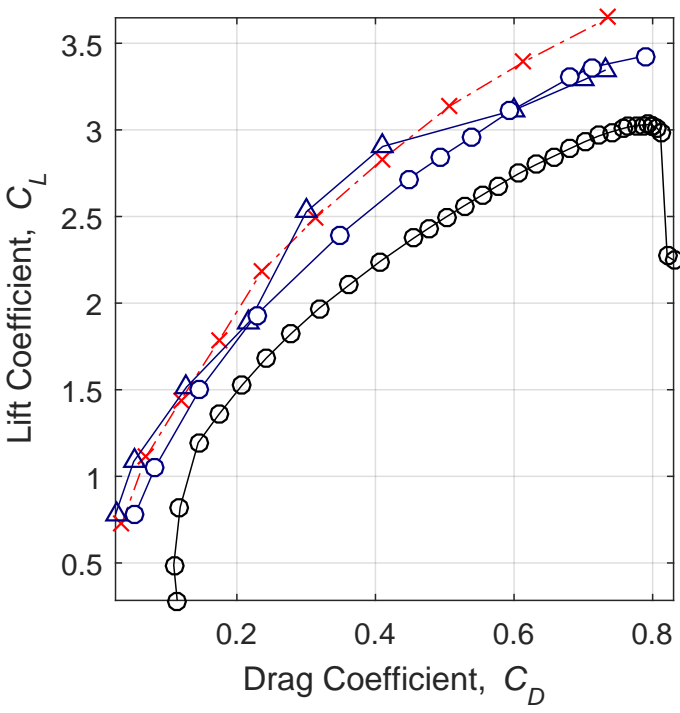

(a) Drag Polar.

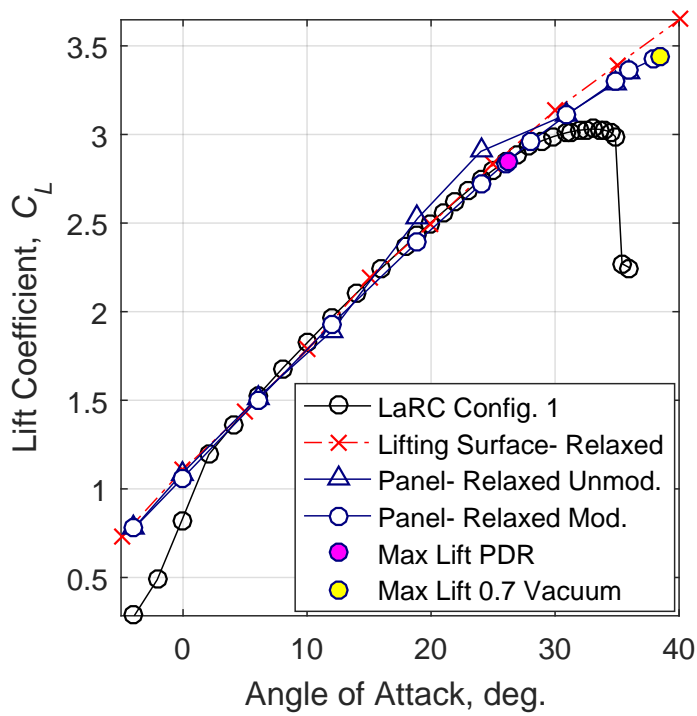

(b) Lift Curve.

Figure 4.16: Aerodynamic predictions of the NASA Trap Wing LaRC Config. 1 using a panel method with a relaxed wake.

the observation made when using a fixed wake approach. The predictions of maximum lift using the modified relaxed wake are shown in Fig. 4.16b. These predictions are similar to those made using a fixed wake approach; the pressure difference rule predicts the maximum lift coefficient to within 0.178 , and the 0.7 vacuum relation finds the maximum angle of attack to within 3.7 degrees. These results were found running the panel method in parallel with two cores on a personal computer using an Intel Core i7 processor capable of running at $2.9 \mathrm{GHz}$. With this computational performance increase, the results for each angle of attack analysis were found in 22 minutes, approximately the same amount of time as the results found with a fixed wake.

To further explore the maximum lift predictions found using the modified relaxed wake, the results of the pressure difference rule study are plotted across the span of each wing in Fig. 4.17. In each figure, the wing root is denoted as section ' 0 ' and the wing tip is section ' 9 '. Note how the pressures over the main wing are well away from the maximum pressure difference across the entire span. Most of the flap span is also well protected from stall, except at the wing tip where the leading edge pressure peak on the flap is very high. This increase in the minimum pressure is not seen when modeling the system using a fixed wake. This leads to the conclusion that the roll-up at the wing tip of the main wing's wake has 
a large impact on the pressures on the upper surface of the flap. When the wake elements are rolled up, they move further away from the flap surface, thereby inducing smaller downwash velocities and allowing for lower pressures on the upper surface of the flap.

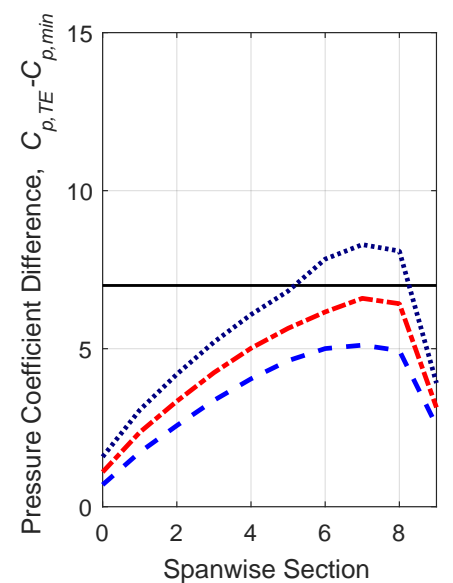

(a) Slat.

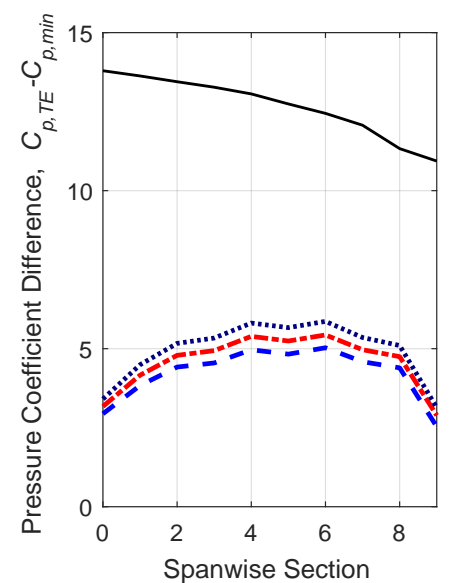

(b) Main Wing.

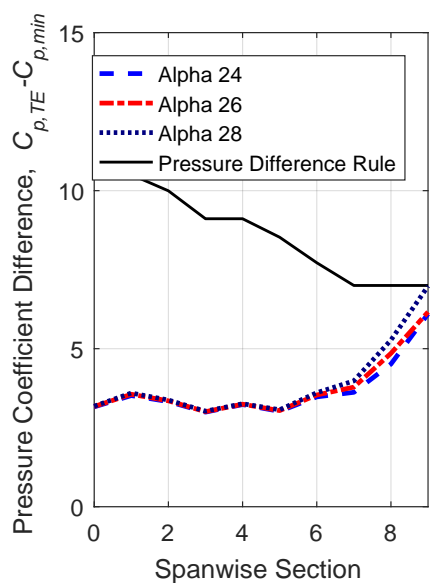

(c) Flap.

Figure 4.17: Predictions of the difference between the minimum pressure peak and trailing edge pressure in the spanwise direction for the NASA Trap Wing LaRC Config. 1. Each spanwise section corresponds to $10 \%$ of the halfspan.

The maximum lift predictions for the slat, seen in Fig. 4.17a, reach the maximum pressure difference at an angle of attack of 26 degrees inbetween $70 \%$ and $80 \%$ of the halfspan. This critical semi-span location on the slat is similar to that of $80 \%$ as found by the Boeing team in the AIAA CFD HighLift Prediction Workshop (HiLiftPW-1) [30]. It must be noted that the pressure difference rule is not intended predict the stall angle, rather, it is meant to provide the maximum lift coefficient $[2,17]$. It does this relatively well, with an error of $6 \%$.

Using the 0.7 vacuum relation, as shown in Fig. 4.18, predicts the maximum angle of attack to within roughly $10 \%$. This extrapolated result is plausible since the 0.7 vacuum relation is meant to provide the angle of attack when the minimum pressure coefficient occurs [7], which can happen at angles of attack larger than when the maximum lift coefficient is reached. Similar to the results found with the pressure difference rule, the 0.7 vacuum relation finds the critical location inbetween $70 \%$ and $80 \%$ of the halfspan of the slat, and the main wing and most of the flap are well protected from stall. Any further conclusions made from the results are only speculative, since viscous effects are not captured by the panel method. 


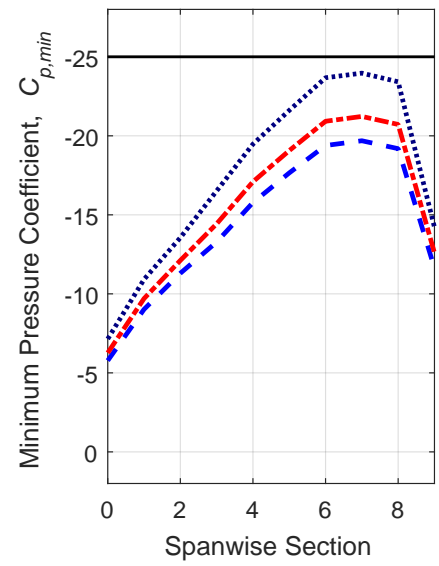

(a) Slat.

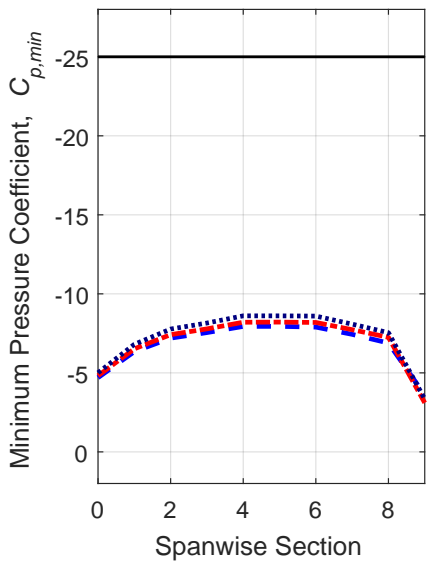

(b) Main Wing.

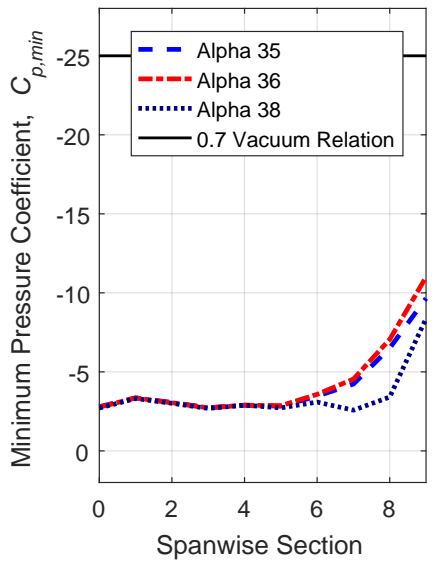

(c) Flap.

Figure 4.18: Prediction of the minimum pressure peak value in the spanwise direction for the NASA Trap Wing LaRC Config. 1. Each spanwise section corresponds to $10 \%$ of the halfspan.

Of course, both the pressure difference rule and the 0.7 vacuum relation rely on a good prediction of the surface pressures. Two examples of the chordwise pressure distributions as found with the panel method using the modified relaxed wake are shown in Fig. 4.19. Shown by the black circles are the available pressures from the wind-tunnel tests. The predictions at both $41 \%$ and $65 \%$ of the halfspan show good agreement with wind tunnel data for the slat, and decent agreement to the experimental data for the flap. Over the main wing, the upper-surface pressures are insufficiently low while the lower-surface pressures are too high. This does not affect the predictions of the pressure difference rule, however, since the difference between the leading edge and trailing edge pressures are similar to the wind-tunnel results. Additionally, as mentioned, the maximum lift is assumed to be driven by the slat and not the main wing.

It must be noted that these pressures, and the pressures used in the maximum lift prediction methods, were found by smoothing the actual pressures predicted by the panel code. This involved a running average of the values to remove most of the waviness introduced by the triangular elements. Even with smoothing, it can be seen that irregularities exist near the trailing edge of each lifting surface. Therefore, the second-to-last values were used in the calculation of the pressure difference rule. 


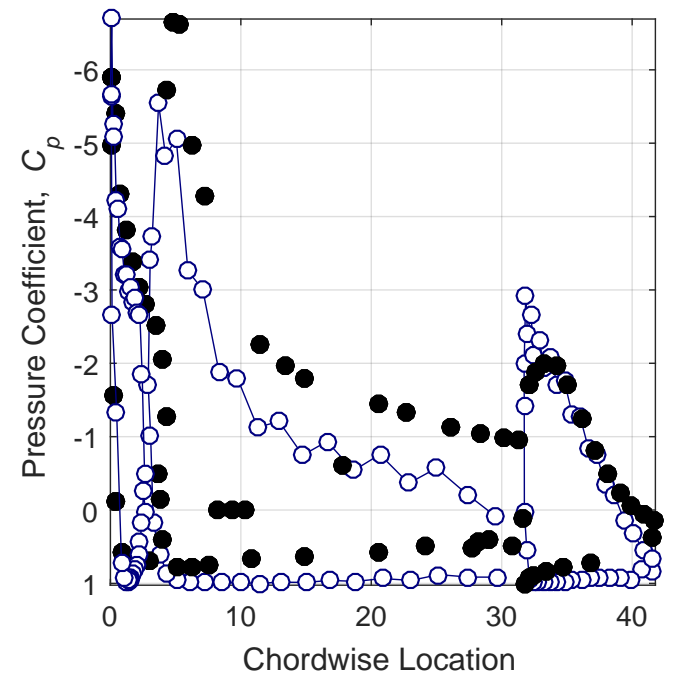

(a) $\operatorname{Eta}=41 \%$

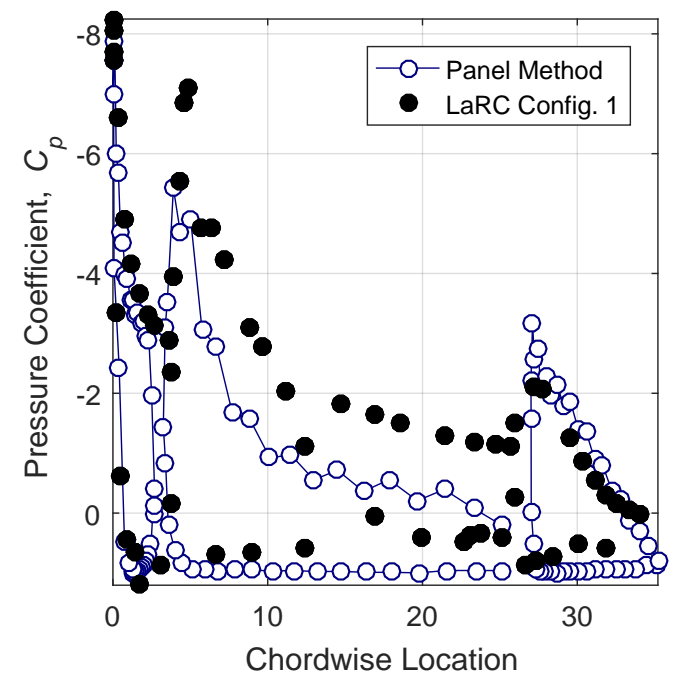

(b) $\mathrm{Eta}=65 \%$

Figure 4.19: Surface pressure predictions of the NASA Trap Wing LaRC Config. 1 at an angle of attack of 24 degrees using a modified relaxed wake.

\subsection{Summary}

The surface pressures found with the developed panel method allow for a prediction of the maximum lift capabilities of a high-lift system. As was described, they are based on theoretical formulas and wind-tunnel observations to form a set of limits based on flow parameters. The pressure difference rule of Valarezo and Chin is able to predict the maximum lift coefficient to within roughly $6 \%$ of the windtunnel data, and J.P Mayer's 0.7 vacuum pressure relation is able to predict the angle of attack when the minimum pressure coefficient occurs to within roughly $10 \%$. Both methods predict that the critical location is inbetween $70 \%$ and $80 \%$ of the slat's span, the same prediction as modern CFD methods [30]. Without a viscous boundary layer representation, these theoretical methods are the most advanced for determining the maximum lift coefficient, and were relatively easy to implement in the developed panel method.

Even with more advanced solvers, the prediction of maximum lift is difficult, and depends largely on the geometry. Since designs change rapidly in the conceptual design phase, these quick, theoretical limits can be used to predict the maximum lift, and, as this study found, the predictions of maximum lift compare well wind-tunnel data. 


\section{Chapter 5}

\section{Analysis of Wake Shapes}

The analyses presented in Chapters 3 and 4 indicate that the choice of wake model clearly impacts the aerodynamic predictions. To further explore this trend, and to better understand the issues related to the wakes, a study was completed to find the advantages and disadvantages of using different wake shapes to analyze high-lift devices [41]. This chapter summarizes the results of the study.

As mentioned, modeling the shear layer in the wake is used to also enforce the Kutta condition along the trailing edge of a wing. The vorticity shed at this location defines the strength of the steady-state wake, which contributes to the induced velocities used to determine the surface singularity strengths. For lower-order potential flow models, such as traditional vortex lattice methods, the wake is often represented using vortex filaments $[12,18,19]$. Relaxing the wake model by moving it with the local velocities generates a wake with zero flow normal to the wake vorticity, which does not support any forces. Due to the mathematical nature of vortex filaments, however, modeling complex wakes and lifting systems can become challenging and may require the manual placement of the wake in order to achieve a viable solution. In such cases, the wakes may not be aligned with the local streamlines and the wake vorticity will generate lift and drag forces. Furthermore, since the wake induces velocities on the lifting surfaces, modifying the position of the wake will impact surface circulation distribution depending on the wake type used. In this chapter the influence is discussed that different wake models have on the aerodynamic forces developed by their corresponding lifting surfaces. 


\section{$5.1 \quad$ Singularity Issues}

Modeling multiple lifting surfaces with discrete vortex filaments can be numerically difficult due to the singular nature at the center of such filaments. This becomes a problem when a trailing filament is positioned close to a downstream numerical control point, as shown in Fig. 5.1. The resulting large velocities that the singularity element induces can cause computational issues as well as result in an unrealistic flow situation. This problem worsens if the wake is relaxed and filaments in the wake come within close proximity of one another. Because of the large velocities that they induce on one another, the filaments can experience relatively large displacements. As a result, the relaxation process can become erratic.

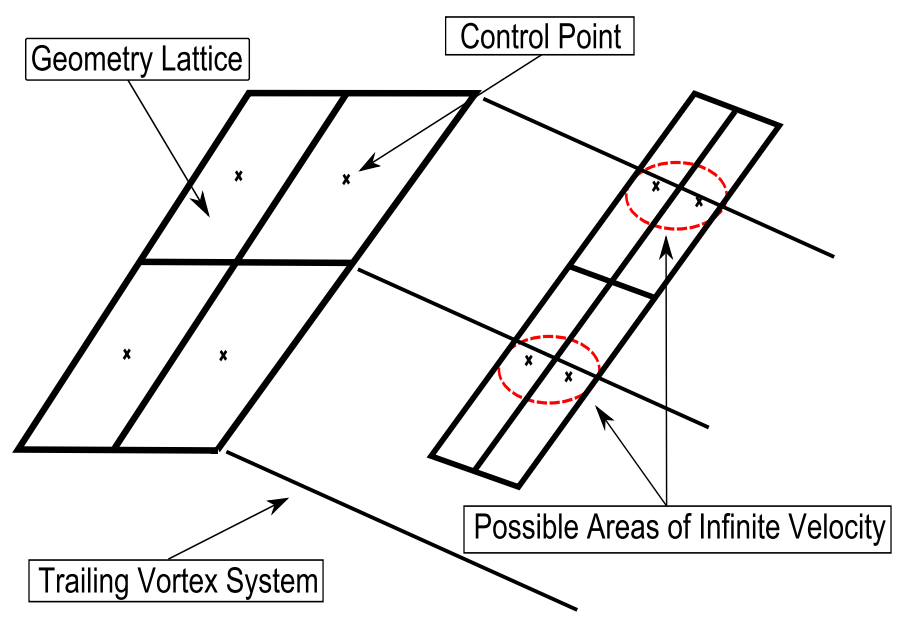

Figure 5.1: A wing and trailing-edge flap system modeled using a traditional vortex lattice method. The discrete filaments used to model the wake shed from the main wing pass closely to the flap, resulting in large velocities at the control point locations.

Multiple methods have been developed to deal with the behavior of the filaments. For example, the numerical issues can be eliminated with use of a solid core model; however the size of the core can easily become a driving factor of the solution. Some potential flow methods offer damping factors to limit the movement of the wake during the relaxation process, and some methods include near field velocity corrections to smooth out velocity peaks near discrete filaments by replacing them with multiple vortex lines of lesser strength distributed over panel edges $[18,19]$. In the case of Ref. 43 , lifting surfaces had to be removed altogether to reduce wake/surface interaction issues. 
Depending on the potential flow solver used, the user may have to manually define the wake position. In some cases, this can lead to geometry simplifications, such as those done during the analysis discussed in Ref. 25. As shown in Fig. 5.2, leading and trailing edge spanwise discontinuities had to be treated; the triple slotted flap geometry was merged into a single slotted flap, and the wakes from each element were prescribed parallel to the downstream surface. With these careful modifications, it was found that the simplifications did not compromise the results [25].

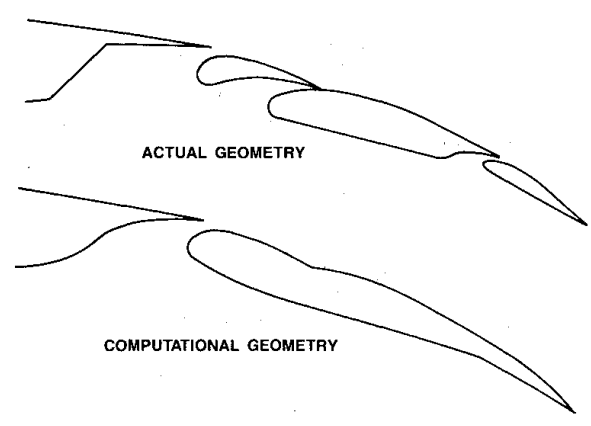

(a) Geometry merging.

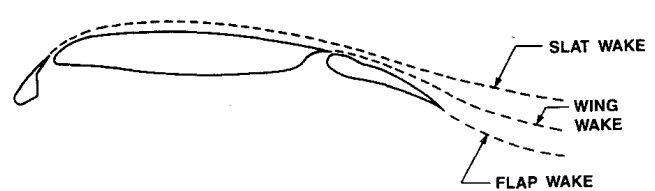

(b) Wake prescribing.

Figure 5.2: High-Lift system modeling simplifications. Adapted from Ref. 25.

For this study, the higher-order potential flow method that is described in Chapter 3 is used to model the lifting surfaces and their wakes. Since this model uses elements of continuous vorticity instead of discrete vortex filaments to model the wake, the numerical issues, as described above, are largely eliminated without the use of a solid core model or manual wake modifications.

Figure 5.3 shows the wake induced downwash velocities along three lines in a plane perpendicular to the wake downstream of a wing. The center figure shows the location of the vortex filaments of a traditional vortex lattice method, shown by the black circles. These filaments induce a discontinuous downwash velocity distribution that is shown by the dashed line. Although these discontinuities can be joined with the use of a solid core model, the method still incorrectly predicts upwash in the location directly behind the wing, which is in contrast to experimental observations and is a nonphysical effect. Alternatively, the continuous vorticity wake of the higher-order method described in Chapter 3 is represented by the blue horizontal line. The downwash velocities induced by this method are shown by the solid blue line. Without any intervention, the curve is continuous and the method correctly predicts only downwash velocities behind the wing and upwash on either side beyond the wingtips. 
The upper and lower plots in Fig. 5.3 show the velocities induced along a line one quarter chord above the wake and one quarter chord below the wake, respectively. At these locations, both of the velocity distributions are continuous, however the velocities induced by the vortex filaments are wavy, especially near the edge of the wake where the strength of the filaments is highest. This nonphysical effect is eliminated when modeling the wake using vortex sheets; the vortex sheet induced velocities are smooth at all locations surveyed.
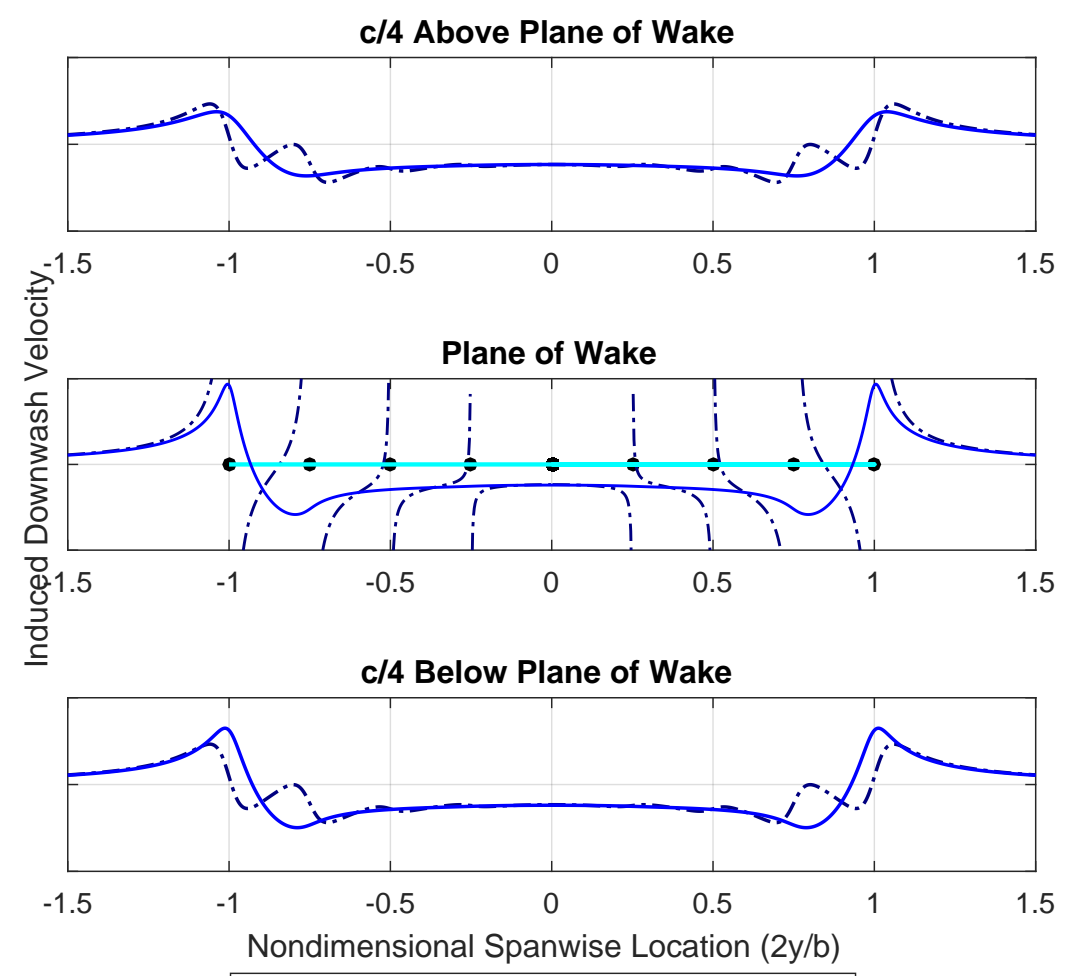

- Vortex Filament Location

Vortex Sheet Location

-.-・Velocity Induced by Vortex Filaments

— Velocity Induced by Vortex Sheet

Figure 5.3: The velocities induced by the wake of a traditional vortex lattice method, as well as a higherorder continuous vorticity method, along three lines on a plane perpendicular to the wake. The lower inset shows the location of the plane where the velocities were found. 


\subsection{Wake Induced Velocities}

Historically, the positioning of the wake in a potential flow solution was not of importance; except for unique cases where strong interaction was expected between lifting elements [24, 26]. For most wing-body configurations, aligning the wake with the streamwise direction produced adequate results. However, as shown in Fig. 5.4, even simple geometries can produce an array of results depending on the type of wake chosen for the analysis. In this case, it was found that Wake 'c' provided predictions that showed the best agreement to experimental data.

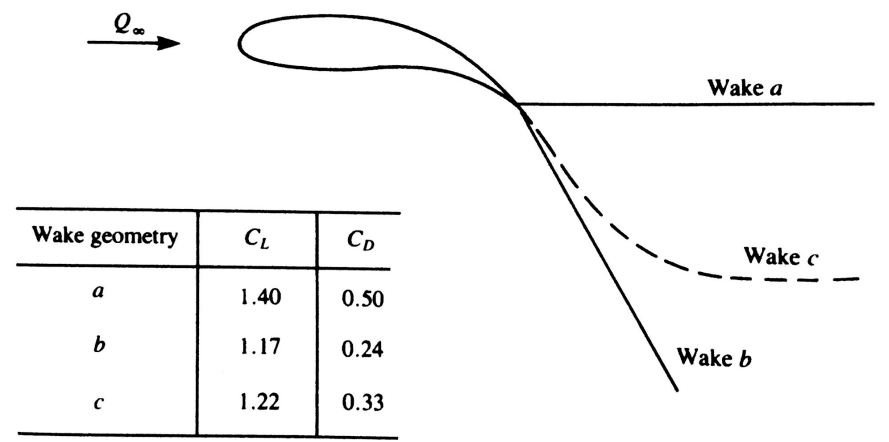

Figure 5.4: Effect of prescribed wake geometries on the aerodynamic predictions of a cambered rectangular wing using a panel method [12].

Multiple-wing configurations have also shown different results depending on the shapes of the wakes. It was found by Miller and Youngblood that the predicted lift of a canard/wing aircraft differed when the wake shed by the canard was positioned in the wing-canard mean plane, rather than aligned with the freestream direction or allowed to roll up. Ultimately, the latter two models produced results which most closely aligned with wind-tunnel testing [44]. A more in depth study of the wake of a canard was performed by Madson and Erickson [45], where they compared predictions using the two wake shapes shown in Fig. 5.5a. They found that a freestream fixed wake produced a 5\% increase in the lift curve slope over a body fixed wake, and the freestream fixed wake produced results much closer to test data. Figure 5.5b shows the resulting spanwise circulation distributions. It was found that the wake model did not have a large effect on the circulation strength of the canard, rather, it had an important effect on the lift distribution along the main wing. Using a freestream fixed wake increased lift inboard of the canard tip station, due to the diminished downwash field produced by the canard wake at that location. Conversely, the outboard sections of the main wing had less lift with a freestream fixed wake, since the 
upwash field was also diminished.
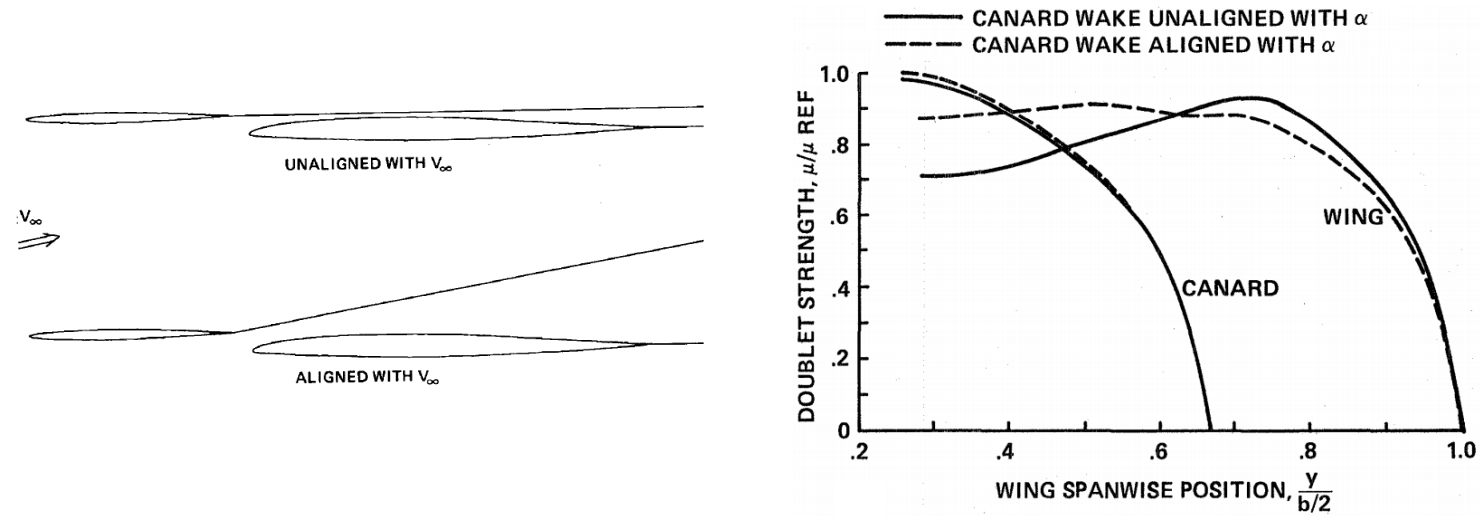

(a) Body fixed (unaligned) and freestream aligned (drag (b) Spanwise circulation distributions using a panel free) wake geometries. Adapted from Ref. 24. method [45].

Figure 5.5: Two prescribed wake models used to analyze a canard-wing fighter concept at an angle of attack of 10 degrees.

Because the higher-order potential flow method of Chapter 3 has none of the singularity issues that are otherwise typical for traditional vortex lattice methods, it is a useful tool for a study of wakes and their relative positions and the subsequent impact on the aerodynamic force predictions. The method of finding the induced drag along the trailing edge is also beneficial since the effects on the induced forces from a wake shed by an upstream surface are captured in this study. In addition, the method of calculating induced drag is less sensitive to changes in the relaxed, rolled-up wake shape when compared to the Trefftz plane drag calculation [22].

\subsection{Comparison of Wake Shapes}

\subsubsection{Single Rectangular Wing}

To analyze the effects of wake shapes on a single lifting surface, and to validate the use of this model for this study, a rectangular wing with a symmetrical airfoil shape and an aspect ratio of 10 was analyzed with the distributed vorticity element lifting surface method. A convergence study was completed on the number of elements used to model the lifting surface for each type of wake used, similar to the convergence study presented for the NASA Trap Wing in Chapter 3. Convergence of span efficiency was 
found when using 8 spanwise elements and 8 chordwise elements, regardless of the wake shape. This discretization was used to model the rectangular wing halfspan for the rectangular wing study. From this convergence study, a simple conclusion can be drawn that the wake shape used to analyze a single, high aspect, rectangular wing does not affect the convergence of the analysis results as the number of elements are increased. This means that, for this study, the same configuration can be modeled with multiple wake types without having to change the discretization of the surface to achieve convergence.

The size of the wake to be used for the study that is described in this section was selected based off convergence of the lift, induced drag, and span efficiency, as the wake grew in length during the time stepping method. Executing 20 timesteps produced converged results for all wake types used. Therefore, this number of timesteps was chosen for the remainder of the study on the rectangular wing. The wing advances one quarter of the root chord length every time step, which also determines the streamwise dimension of the wake elements. Subsequently, the converged solution has a wake which is five times the wing root chord length in the streamwise direction. Since convergence of the span efficiency as the wake grows is independent of the wake type for this single rectangular wing, the same configuration can be modeled with multiple wake types for this study, without having to change the size of the wake to achieve convergence.

Plots of the five wake shapes tested can be seen on the rectangular wing halfspan in Fig. 5.6, for an analysis at an angle of attack of 6 degrees. Note that the prescribed angles are with respect to the zerolift line, since the airfoil is symmetrical. As such, an analysis performed using a freestream fixed, drag free wake at an angle of attack of 10 degrees, for example, produces the same result as an analysis with a wake prescribed to 10 degrees. Also note that the wake elements generated during the first timestep, the row of wake elements furthest from the wing, are fixed to the freestream direction, rather than the prescribed wake angle. This is a result of the time stepping method. During the first timestep, the wake is fixed to the freestream direction to remove large induced velocities that may exist since the system is far from convergence. After convergence is found, the first row of wake elements has moved far enough downstream that the effect of the incorrect wake angle is assumed to be negligible. 


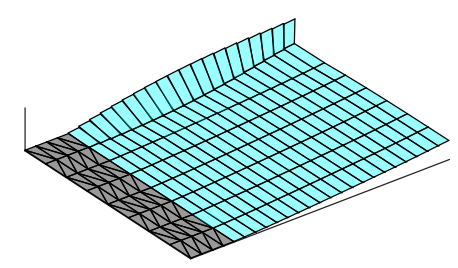

(a) Relaxed.
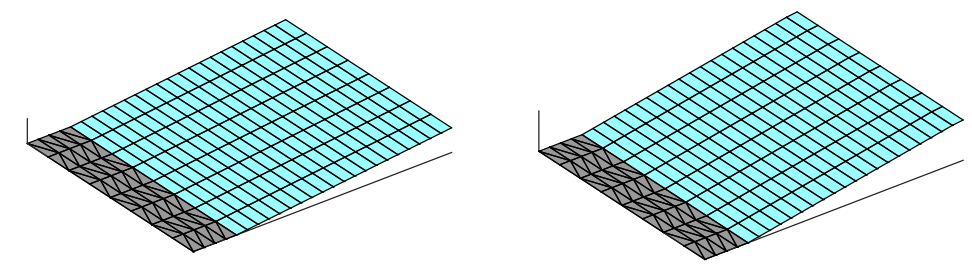

(b) Drag free / freestream fixed.

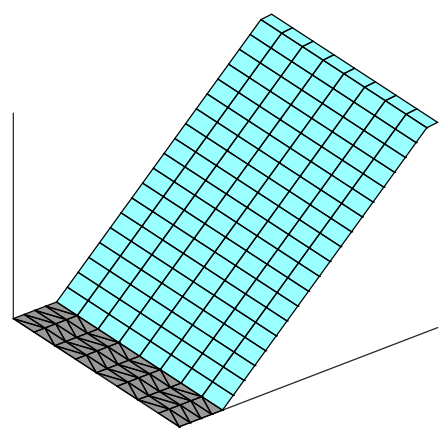

(c) Prescribed to 10 degrees.

(d) Prescribed to 45 degrees.

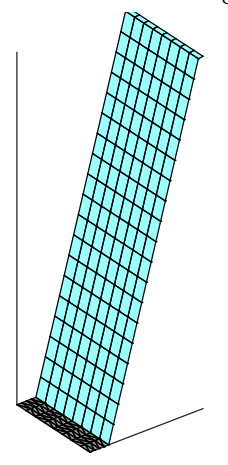

(e) Prescribed to 80 degrees.

Figure 5.6: Wake shapes analyzed on a rectangular wing with an aspect ratio of 10 at an angle of attack of 6 degrees.

The lift curves and drag polars for results obtained using a relaxed wake, a drag free wake, and wakes prescribed to 10, 45 and 80 degrees above the zero-lift line are plotted in Fig 5.7. The results predict very little differences in the lift and induced drag for all wake shapes except for the results with wakes that are prescribed to extreme angles. 


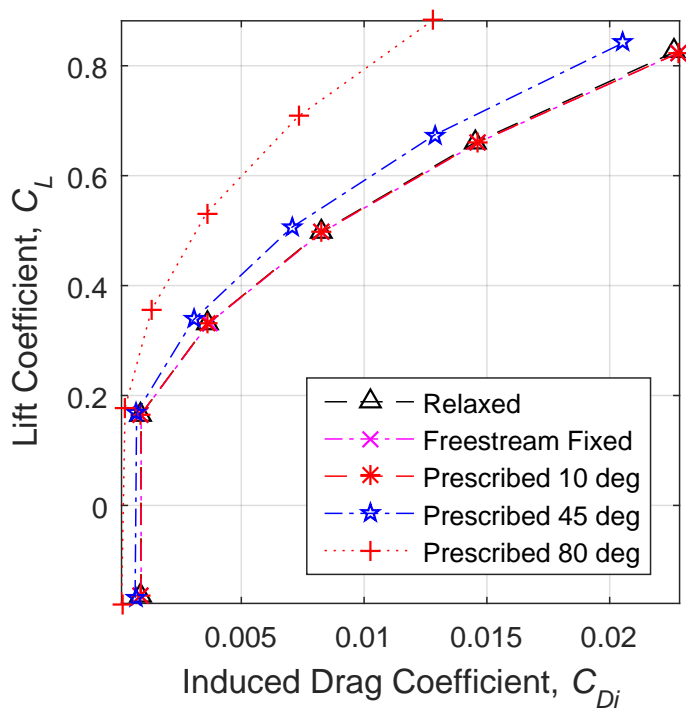

(a) Drag polar.

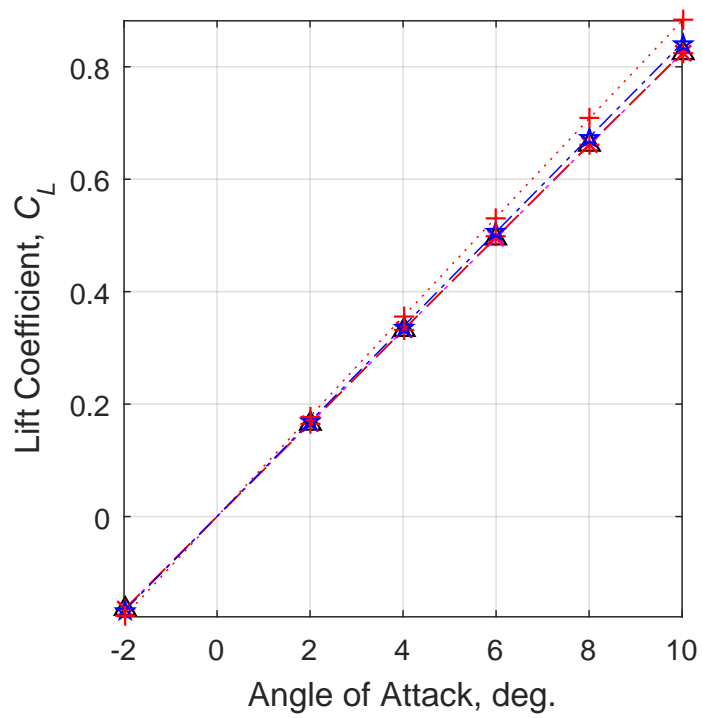

(b) Lift curve.

Figure 5.7: Comparison of lift and induced drag for five different wake shapes for a rectangular wing with an aspect ratio of 10 .

As shown in Fig. 5.8, with high positive wake angles, the velocity induced by the wake on the wing has a large component in the freestream direction. This results in an increase in the predicted lift when comparing large wake angles to drag free wakes. Simultaneously, the component of the wake induced velocity normal to the freestream direction is reduced, resulting in a smaller angle of attack reduction when compared to a freestream fixed wake. Thus, the predicted induced drag is decreased as the wake angle increases. An interesting side note is that a wake prescribed at positive 90 degrees to the freestream will produce zero induced drag with the method. Additionally, although not tested in this study, a wake prescribed below the freestream direction will decrease lift, since the incoming flow velocity is reduced by the wake induced velocity. This will also produce less induced drag than a freestream fixed wake. Therefore, a freestream fixed wake will produce the most induced drag for a given lift coefficient, for a single wing configuration. These correlations between prescribed wake shapes and aerodynamic predictions are also applicable to the relaxed, force-free shapes. They are less intuitive, however, the effects are applied to each element's plane, rather than the plane of the entire wake. 


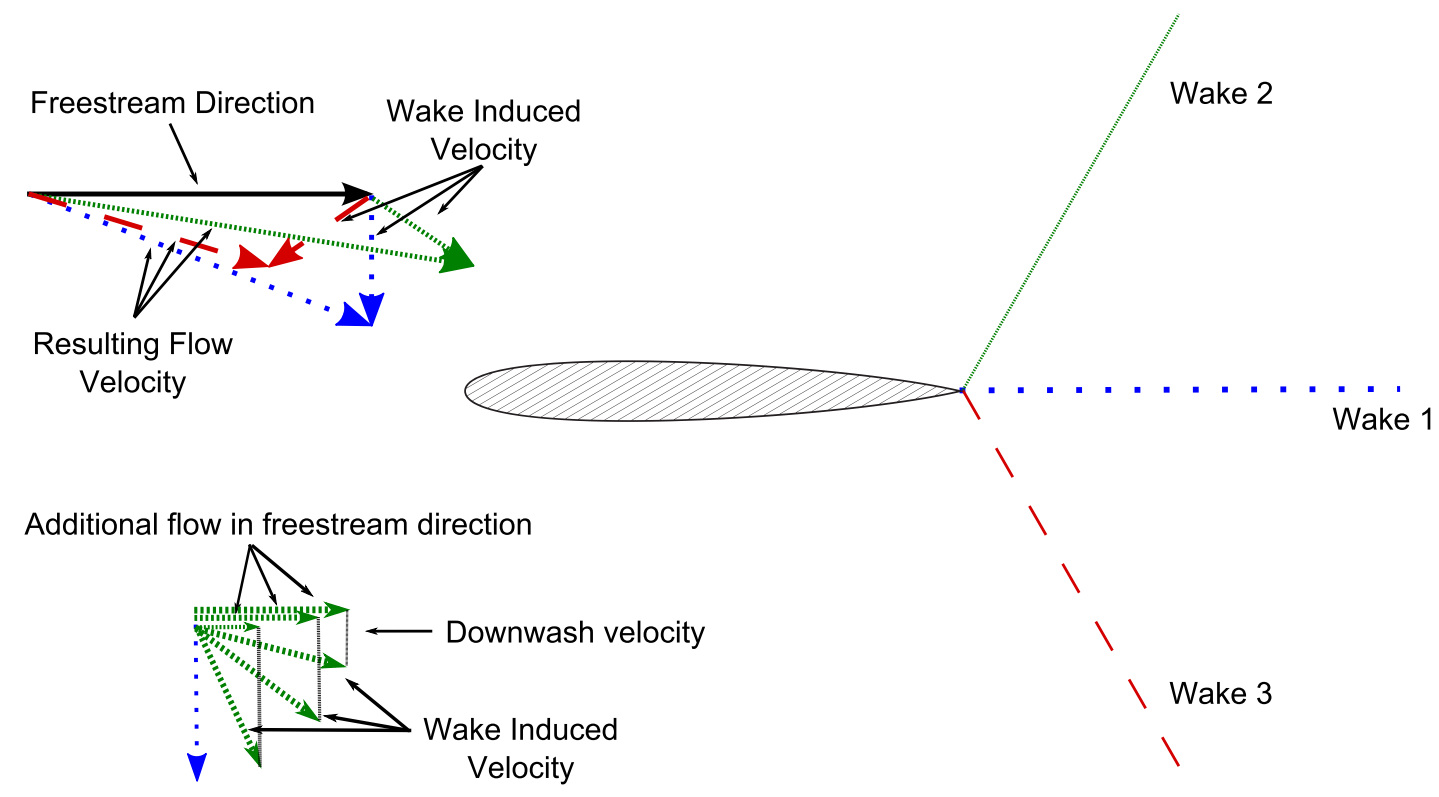

Figure 5.8: Effect on incoming flow velocity due to three prescribed wake shapes of equal strength. Wake 1 is aligned with the freestream (drag free), wake 2 is prescribed above the freestream (positive), and wake 3 below the freestream (negative). The lower inset shows the additional component of the resultant flow vector in the freestream direction as a wake is prescribed to increasing positive wake angles.

\subsubsection{NASA Trap Wing}

To study the effects of wake shapes on a high-lift configuration, the analysis of the NASA Trap Wing LaRC Config. 1, presented in Chapter 3, was extended to include predictions using more wake shapes. The convergence studies presented in Chapter 3 were also extended to ensure convergence was met using all wake types.

For the freestream fixed and all prescribed wake cases, good convergence was seen when 10 elements were used in the spanwise direction. In contrast to the results of the rectangular wing study, the span efficiency convergence when using a relaxed wake becomes more erratic when modeling multiple lifting surfaces with multiple wakes. This is due to the changing geometry of the relaxed wake shape as the number of spanwise elements is increased. Nonetheless, using more than 10 spanwise elements and 12 elements in the chordwise direction produced results that were deemed accurate enough for this study on wake shapes. For further studies, it is suggested that more elements should be used when modeling with a relaxed wake when compared to models using prescribes wake shapes. 
The size of the wake used for this study was based off the convergence of lift, drag, and span efficiency, as the wake grew in length. Similar to the convergence study presented in Chapter 3 and the rectangular wing study, convergence was found when the number of timesteps was equal to 20. This corresponds to a wake length which is five times the root chord length in the horizontal direction. Since this trend continued for all wake shapes used, this wake size was used for this wake shape study on the NASA Trap Wing.

The seven wake positions corresponding to a relaxed wake, a freestream fixed (drag free) wake, and wakes prescribed to $-45,-10,10,45$ and 80 degrees are shown in Fig. 5.9. Since the wing is not symmetrical, a relative axis must be chosen for the prescribed wake directions. This has been chosen as the global XY axis, which corresponds to the freestream direction at an angle of attack of zero degrees. This means that an analysis at an angle of attack of 10 degrees with a freestream fixed wake would produce a wake which is identical to a wake prescribed to 10 degrees.

Figures 5.10 and 5.11 compare the results of the study to the experimentally obtained NASA Trap Wing LaRC Config. 1 data. As was the case for the analysis of the NASA Trap Wing presented in Chapter 3, no viscous model is incorporated into the current analysis method. Hence, only the linear region of the lift curve is being compared, and the predicted drag values are solely induced drag, whereas the experimental drag values are total drag. Therefore, an offset in drag is expected, and only the general shape of the polars are being compared.

As was found with the rectangular wing study, the freestream fixed wake produces the most induced drag for a given lift coefficient. This value then decreases as the wake angle is tilted. The reason that the drag produced by negative wake angles decreases above a certain lift coefficient is not fully understood. Zero induced drag should be predicted by a wake prescribed 90 degrees to the freestream direction, however, this does not seem to be the case for negative wake angles. In addition, the intersection points between positive and negative wake angles are not fully understood. For this reason, negative wake angles will be ignored when comparing drag values. 


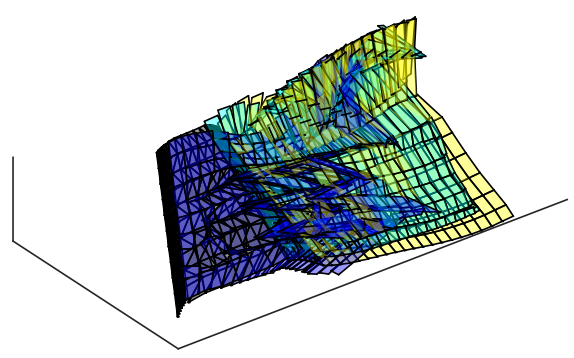

(a) Relaxed.

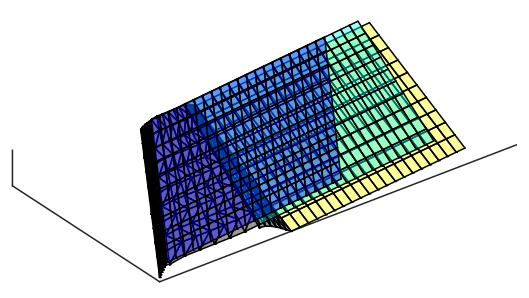

(b) Drag free / freestream fixed.

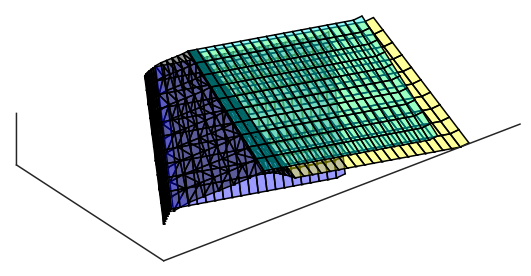

(d) Prescribed to negative 10 degrees.

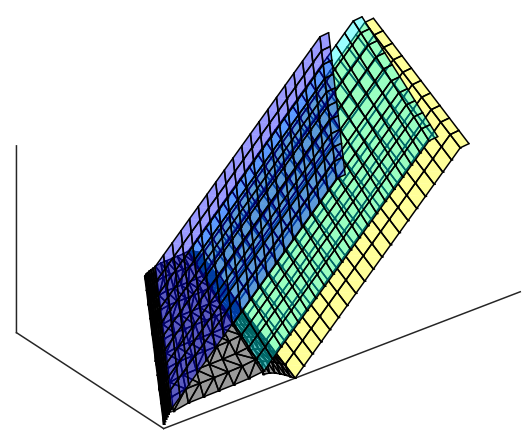

(f) Prescribed to 45 degrees.

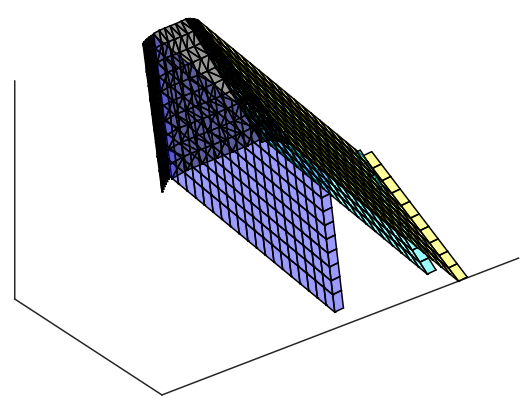

(c) Prescribed to negative 45 degrees.

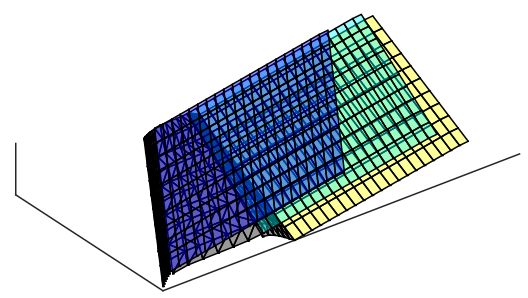

(e) Prescribed to 10 degrees.

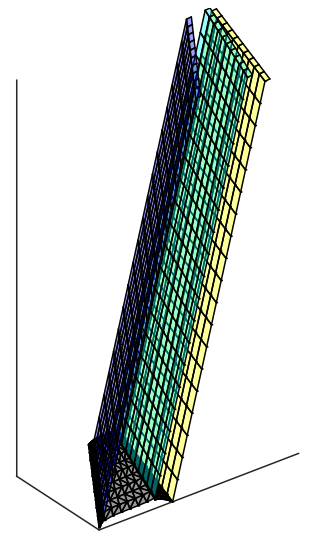

(g) Prescribed to 80 degrees.

Figure 5.9: Wake shapes analyzed on the NASA Trap Wing LaRC Config. 1 at an angle of attack of 5 degrees. 


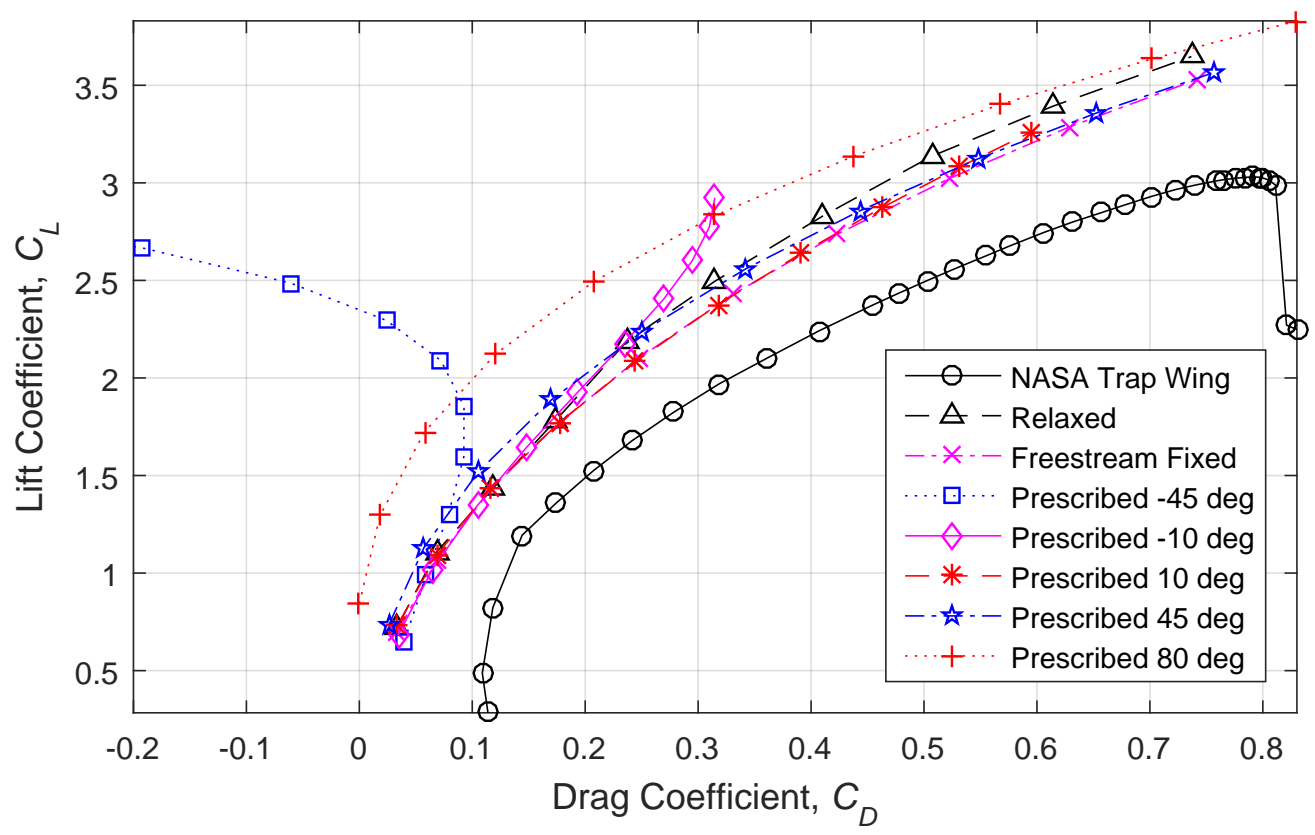

Figure 5.10: Drag predictions for the NASA Trap Wing LaRC Config. 1 using different wake types.

As was previously mentioned, a wake prescribed to the freestream angle produces the same aerodynamic predictions as a freestream fixed wake. At lower and higher freestream angles, the prescribed wake will predict lower induced drag. Therefore, the predicted drag polars will not be of the same shape, as shown in Fig. 5.10. As a result, for a given lift coefficient, wakes prescribed to angles less than 10 degrees from the freestream angle of attack produce very similar results to the freestream fixed wake.

As can be seen in Fig. 5.11, the major difference between the predicted lift coefficients is the lift curve slope. When a wake is prescribed above the freestream direction, additional lift is predicted. The opposite is true for wakes prescribed below the freesream direciton. At a given angle of attack, lift coefficients will deviate approximately $2.7 \%$ for every 10 degree difference between the prescribed wake angle and the freestream direction. This is due to the combination of two effects. The first effects is that the wake of a lifting surface affects the incoming flow vector. This is in line with the observations that were made with the single rectangular wing analysis, and is depicted in Fig. 5.8. The second is effect is unique to cases with multiple lifting elements. Since the downwash velocities are highest in the plane of the wake itself, prescribing a wake to a defined angle will affect the strength of the downwash field that a subsequent lifting surface passes through. The resulting spanwise circulation distributions will be 


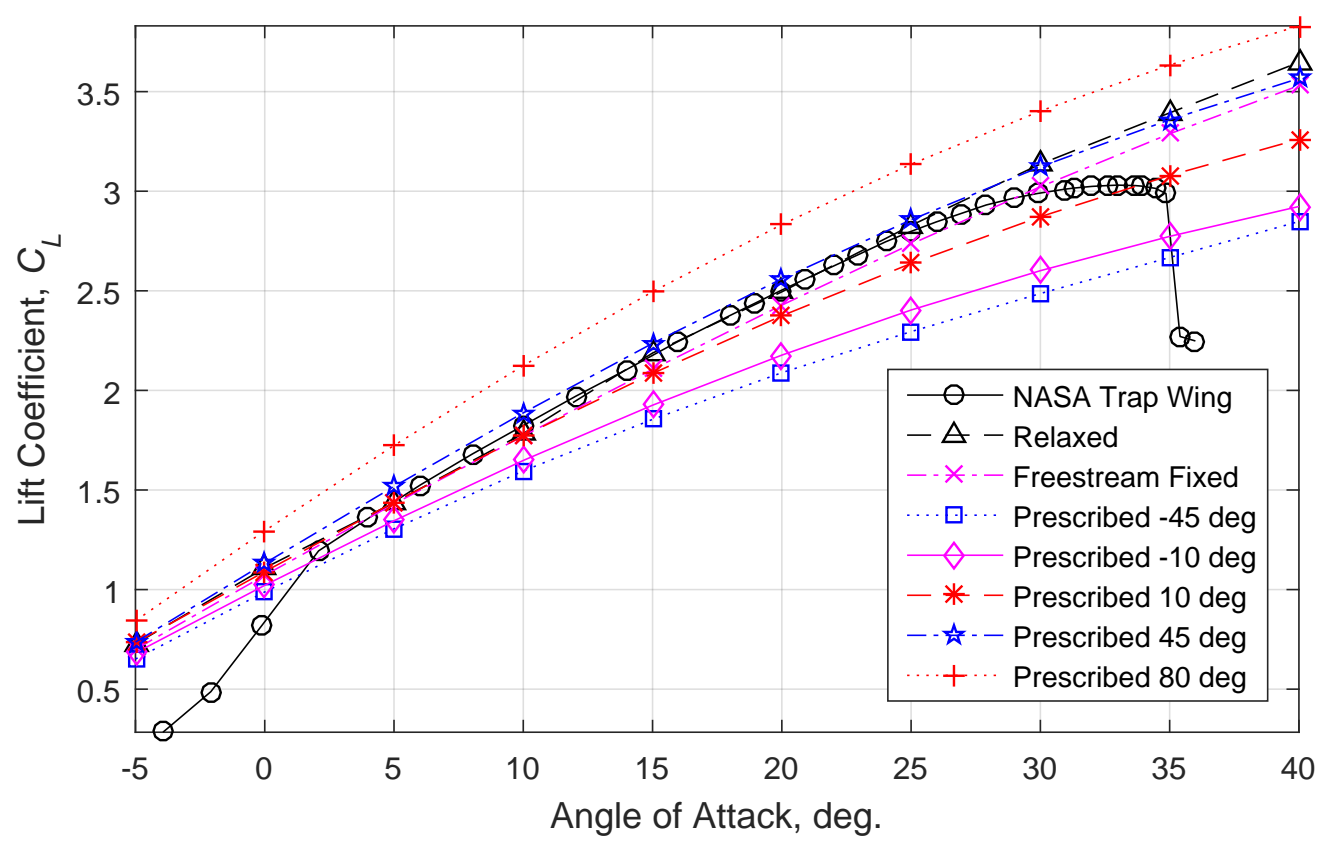

Figure 5.11: Lift predictions for the NASA Trap Wing LaRC Config. 1 using different wake types.

affected, as shown for multiple wake types in Fig. 5.13. Since the trailing edge of the slat and main wing shed their wakes on top of the subsequent lifting surfaces, prescribing a wake angle to any angle above or below the subsequent lifting surface decreases the downwash at that location, therefore leading to an increase in the lift of the downstream element. Alternatively, prescribing a wake that passes through a downstream lifting surface results in large downwash velocities at the downstream location.

It is a result of the first effect described above, the change in inflow direction due to the close proximity of a wake, which causes the lift curves to be non-linear when prescribed wakes are used. As shown by the lower inset in Fig. 5.8, as the wake induced velocity vector is tilted counter-clockwise, the additional velocity it produces in the freestream direction increases with the cosine of the difference between the angle of attack and the prescribed wake angle. As a result, the estimated extra lift reduces as the angle of attack approaches the prescribed wake angle.

The results of this wake shape study on the NASA Trap Wing find the same conclusions that were made during the canard-wing studies by Miller and Youngblood [44], and further explored by Madson and Erikson [45]. Figure 5.11 shows that a relaxed wake provides results closest to experimental data, and a freestream fixed wake will provide more lift at a given angle of attack when compared to a wake 
aligned close to the body. Applying the correction factor derived above to the prescribed wake shapes results in the lift predictions as shown in Fig. 5.12. Each of the predictions show good agreement to the results found when using a freestream fixed wake.

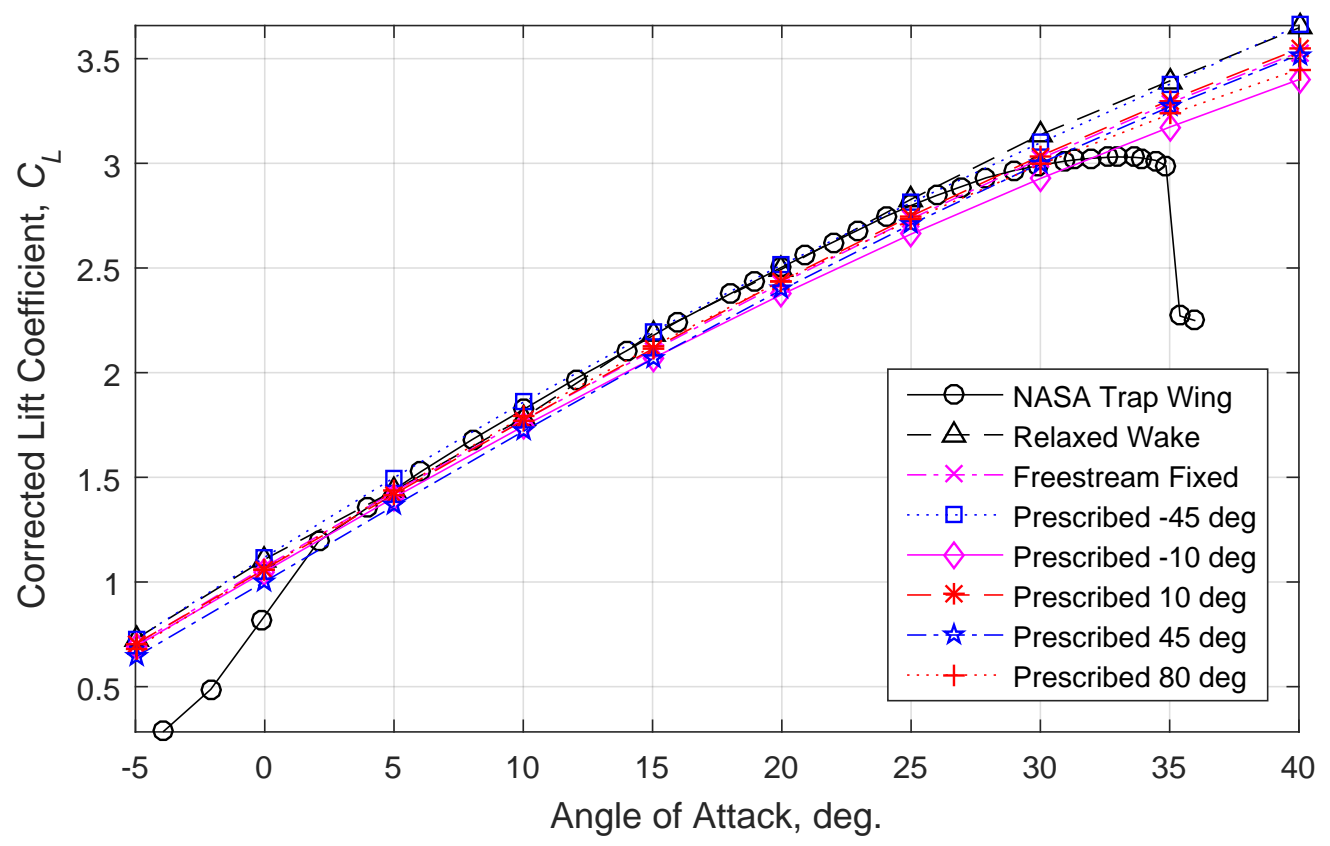

Figure 5.12: Corrected lift predictions. Values are corrected based on the conclusion that lift coefficients deviate $2.7 \%$ for every 10 degree difference between the prescribed wake angle and the freestream direction.

The spanwise circulation distributions shown below are normalized to the circulation at the root of the main wing, as found when using a relaxed wake shape. Negative circulation at the inboard section of the slat produces a net downwards force at that location. This may be physical, due to the nature of the slat, however, it is probably due to the simplifications required to model a complex, three-dimensional surface with an infinitely thin wing. These problems, and the solution taken, are described in Ref. 39.

As expected, the circulation values produced when using a wake prescribed to positive angles are higher than those predicted with a relaxed wake, except at the tip of the flap. This is a result of the relaxed wakes from the slat and main wing being rolled up to angles higher than 80 degrees as they pass over the flap. This reduces the downwash at that location more than the wakes prescribed to high angles. The opposite effect happens with wakes prescribed to angles less than the freestream direction. 


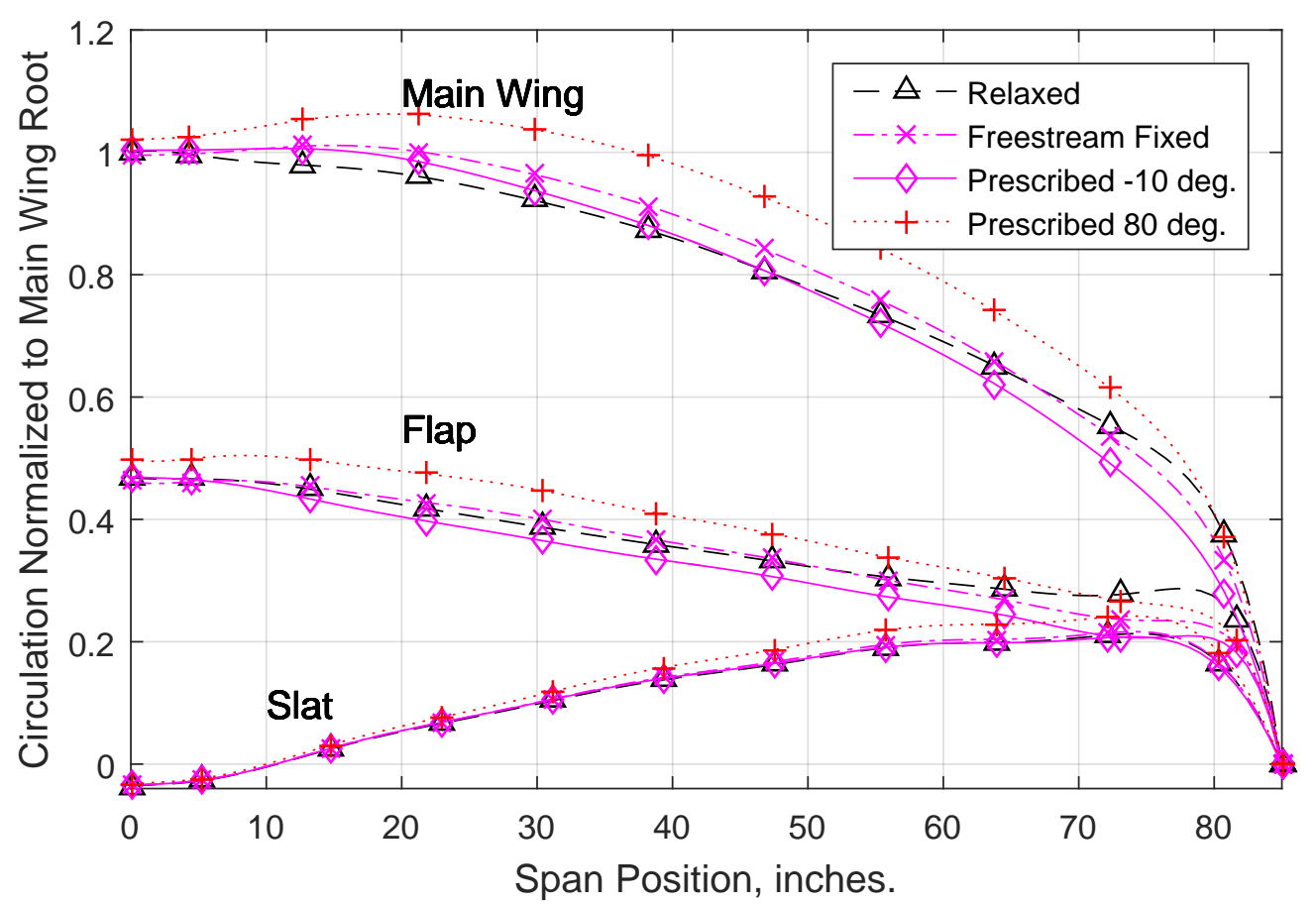

Figure 5.13: Predicted spanwise circulation distributions for the NASA Trap Wing LaRC Config. 1, at an angle of attack of 20 degrees. Values are normalized to the circulation at the main wing root section, as predicted with a relaxed wake.

\subsection{Summary}

The effects of wake shapes on the aerodynamic predictions of multiple wing types were explored using the lifting surface approach of Chapter 3. For a single rectangular wing, convergence of the results is not affected by the wake type. This can save computational effort during an analysis, since the same geometry can be used if different wake types are being modeled. In contrast, an analysis of multiple lifting surfaces requires more elements in the spanwise direction when using a relaxed wake model when compared to prescribed wake models. For both geometries tested, results converged when the wakes measured 5 times of the length of the root chord in the streamwise direction. As such, for this study, the wake length was held constant while the type of wake was modified.

For the single, high aspect ratio wing analysis, very little difference in the induced drag predictions were observed when changing wake shapes, except when using wakes prescribed to extreme angles above 
45 degrees. These results differed when analyzing multiple lifting surface configurations. As described, for a given lift coefficient, the most induced drag will always be predicted by a freestream fixed wake. If prescribed wakes are to be used, they should not be prescribed more than 10 degrees from the freestream direction to reduce error in the induced drag calculation.

Lift coefficients vary little with different wake types when analyzing a single rectangular wing. In contrast, when analyzing multiple lifting surfaces, the lift curve slopes produced with different wake types vary. At a given angle of attack, lift coefficients will deviate approximately $2.7 \%$ for every ten degree difference between the prescribed wake angle and the freestream direction. This correction factor can be applied to the results of prescribed wake shapes to generate values which show good agreement to experimental data.

The results computed with relaxed wake shapes showed the best agreement towards experimentally obtained data. However, this comes at the cost of increased computational effort. A relaxed wake analysis requires approximately $25 \%$ more time to achieve convergence, when compared to a prescribed wake. If a slight reduction in accuracy can be accepted in favor of a shorter analysis time, the recommendations made in this study should be used to reduce errors introduced by the wake prescribing process.

Ultimately, this study on wake shapes has shown that prescribing the wakes to any position, other than the position of a relaxed wake, introduces errors to the solution. Although these errors can be corrected, the method of Chapter 3, which uses distributed vorticity elements to represent the shear layer in the wake, can be used with a relaxed wake shape without any need for manual wake adjustments. This method does not introduce any errors due to the position of the wake. 


\section{Chapter 6}

\section{Conclusions}

This thesis presents a method to analyze high-lift devices early in the conceptual design phase. The method is based on a higher-order lifting surface model, which has been implemented as both a lifting surface method and a full panel method. Both implementations have the numerical robustness of a wake with a continuous vorticity distribution. This allows for a relaxed wake model to be used, and eliminates the singularity issues caused by wake interactions. As such, the method is well suited for an iterative approach where manual intervention is not ideal. These advantages over traditional aerodynamic analysis methods allow designers to more efficiently analyze high-lift systems at the conceptual design stage, when designs change rapidly and efficient aerodynamic predictions are necessary.

\subsection{Lifting Surface Method}

The lifting surface implementation predicts lift and induced drag values that agree well with experimental data. The method is able to predict changes in performance due to geometry modifications as well. The major advantage of the lifting surface method is its computational efficiency; a single analysis can be completed in minutes using a personal computer, compared to hours required by modern CFD solvers, even when using hundreds of computing nodes. This allows for the lift and induced drag predictions of multiple lifting systems to be estimated quickly. 


\subsection{Panel Method}

A higher-order potential flow method was expanded to a panel method. Including the thickness effects allows for the calculation of surface pressure differentials that can be used to predict the maximum lift capabilities of a high-lift system. When using the analytical pressure difference rule of Valarezo and Chin, the predicted maximum lift coefficient of the NASA Trap Wing was found to within $6 \%$. This method also predicted the same critical spanwise location that was found by modern CFD solvers. When applying J.P Mayer's 0.7 vacuum pressure relation, the maximum angle of attack of the system was estimated to within roughly $10 \%$. Both of these prediction methods provide rapid results, allowing for the maximum lift capabilities of multiple designs to be quickly estimated.

\subsection{Wake Shapes}

The errors introduced by a prescribed wake shape on the aerodynamic predictions of a lifting element can be large if the wake is prescribed to angles far from the freestream direction. It was found that, for a single, rectangular wing, the choice of wake type did not affect the convergence of the results as panel density was increased or the wake length grew. In contrast, for a more complex, multiple lifting element system, using a relaxed wake requires a larger number of spanwise elements in order to achieve convergence, compared to when using a prescribed wake model.

When modifying the prescribed wake angle, very little differences were seen in the predictions of the single rectangular wing except for when the wake was prescribed to angles greater than 45 degrees above the horizontal. For the multiple lifting element system tested, noticeable differences were observed with respect to the experiment when the wake was prescribed to angles larger than 10 degrees above the horizontal.

Ultimately, relaxed wake predictions show the best agreement towards experimentally obtained data. However, an analysis using a relaxed wake comes at the cost of increased computational effort. In this study, it was found that a relaxed wake required approximately $25 \%$ more time for convergence when compared to a prescribed wake approach. Nevertheless, compared to typical CFD-based predictions, the higher-order potential flow model required significantly less computational effort, which makes it quite suitable for the conceptual design phase. 


\section{Bibliography}

[1] Rudolph, P. K., "High-Lift Systems on Commercial Subsonic Airliners," NASA CR-4746, Sept. 1996.

[2] Van Dam, C., "The Aerodynamic Design of Multi-Element High-Lift Systems for Transport Airplanes," Progress in Aerospace Sciences, Vol. 38, No. 2, 2002, pp. 101-144.

[3] Flaig, A. and Hilbig, R., "High-Lift Design for Large Civil Aircraft," AGARD CP-515, 1993, pp. $31.1-31.12$.

[4] Nield, B. N., "An Overview of the Boeing 777 High Lift Aerodynamic Design," Aeronautical Journal, Vol. 99, No. 989, 1995, pp. 361-371.

[5] Bissonnette, B. and Bramesfeld, G., "Demonstration of a Conceptual Design Tool for Multiple Lifting Elements," 53rd AIAA Aerospace Sciences Meeting, Vol. 2015-1031, Kissimmee, FL, 2015.

[6] Meredith, P., "Viscous Phenomena Affecting High-Lift Systems and Suggestions for Future CFD Development," AGARD CP-515, 1993, pp. 19.1-19.8.

[7] Smith, A. M. O., "High-Lift Aerodynamics," Journal of Aircraft, Vol. 12, No. 6, 1975, pp. 501-530.

[8] Reckzeh, D., "Aerodynamic Design of the High-Lift-Wing for a Megaliner Aircraft," Aerospace Science and Technology, Vol. 7, No. 2, 2003, pp. 107-119.

[9] Werner-Spatz, C., Heinze, W., and Horst, P., "Improved Representation of High-Lift Devices for a Multidisciplinary Conceptual Aircraft Design Process," Journal of Aircraft, Vol. 46, No. 6, 2009, pp. 1984-1994. 
[10] Sutcliffe, M., Reckzeh, D., and Fischer, M., "Hicon Aerodynamics- High Lift Aerodynamic Design for the Future," 25th International Congress of the Aeronautical Sciences, Hamburg, Germany, 2006.

[11] F.A.R, "Part 25-Airworthiness standards: Transport category airplanes," Federal Aviation Administration (FAA), USA, 2013.

[12] Katz, J. and Plotkin, A., Low-Speed Aerodynamics, Vol. 13, Cambridge University Press, New York, 2001.

[13] Drela, M., Flight Vehicle Aerodynamics, MIT Press, Massachusetts, 2014.

[14] Coder, J. G. and Maughmer, M. D., "Comparisons of Theoretical Methods for Predicting Airfoil Aerodynamic Characteristics," Journal of Aircraft, Vol. 51, No. 1, 2014, pp. 183-191.

[15] Maughmer, M. D. and Coder, J. G., "Comparisons of Theoretical Methods for Predicting Airfoil Aerodynamic Characteristics," Tech. Rep. 10-D-106, U.S Army Research, Development and Engineering Command, 2010.

[16] Strüber, H., "The Aerodynamic Design of the A350 XWB-900 High Lift System," 29th International Congress of the Aeronautical Sciences, St. Petersburg, Russia, 2014.

[17] Valarezo, W. O. and Chin, V. D., "Method for the Prediction of Wing Maximum Lift," Journal of Aircraft, Vol. 31, No. 1, 1994, pp. 103-109.

[18] Maskew, B., "Program VSAERO Theory Document: A computer program for calculating nonlinear aerodynamic characteristics of arbitrary configurations," NASA CR-4023, Sept. 1987.

[19] Ashby, D. L., Dudley, M. R., Iguchi, S. K., Browne, L., and Katz, J., "Potential Flow Theory and Operation Guide for the Panel Code PMARC," NASA TM-102851, Jan. 1991.

[20] Mortara, K. W. and Maughmer, M. D., "A Method for the Prediction of Induced Drag for Planar and Non-planar Wings," 11th Applied Aerodynamics Conference, Vol. 1993-3420, Monterey, CA, 1993, pp. 179-189.

[21] Eppler, R., "Die Entwicklung der Tragflügeltheorie," Zeitschrift für Flugwissenschaften und Weltraumforschung, Vol. 11, No. 3, 1987, pp. 133-144. 
[22] Bramesfeld, G., A Higher Order Vortex-Lattice Method with a Force-Free Wake, Ph.D. thesis, The Pennsylvania State University, 2006.

[23] Drela, M., "XFOIL: An analysis and design system for low Reynolds number airfoils," Low Reynolds number aerodynamics, Springer, 1989, pp. 1-12.

[24] Erickson, L. L., "Panel Methods: An introduction," NASA TP-2995, 1990.

[25] Tinoco, E., Ball, D., and RICE, II, F., "PAN AIR Analysis of a Transport High-Lift Configuration," Journal of Aircraft, Vol. 24, No. 3, 1987, pp. 181-187.

[26] Epton, M. and Magnus, A., "PAN AIR: A computer program for predicting subsonic or supersonic linear potential flows about arbitrary configurations using a higher order panel method. Volume 1: Theory document (version 3.0)," NASA CR-3251, Mar. 1990.

[27] Rumsey, C., Slotnick, J., Long, M., Stuever, R., and Wayman, T., "Summary of the First AIAA CFD High-Lift Prediction Workshop," Journal of Aircraft, Vol. 48, No. 6, 2011, pp. 2068-2079.

[28] Murayama, M., Tanaka, K., and Yamamoto, K., "CFD Comparison Study for Trapezoidal HighLift Wing Configurations by Structured and Unstructured Mesh Method," 49th AIAA Aerospace Sciences Meeting, Vol. 2011-937, Orlando, FL, 2011.

[29] Lee, H. C. and Pulliam, T. H., "Effect of Using Near and Off-Body Grids with Grid Adaption to Simulate Airplane Geometries," 20th AIAA Computational Fluid Dynamics Conference, Vol. 2011-3985, Honolulu, HI, Jun. 2011.

[30] Sclafani, A. J., Slotnick, J. P., Vassberg, J. C., Pulliam, T. H., and Lee, H. C., "OVERFLOW Analysis of the NASA Trap Wing Model from the First High Lift Prediction Workshop," 49th AIAA Aerospace Sciences Meeting, Vol. 2011-866, Orlando, FL, Jan. 2011.

[31] Fares, E. and Nölting, S., "Unsteady Flow Simulation of a High-Lift Configuration Using a Lattice Boltzmann Approach," 49th AIAA Aerospace Sciences Meeting, Vol. 2011-869, Orlando, FL, Jan. 2011, pp. $4-5$.

[32] Ravindra, K., Shende, N. V., Patel, S. K., and Balakrishnan, N., "Unstructured Adaptive Mesh Calculations for NASA Trap Wing Using the Code HiFUN," 30th AIAA Applied Aerodynamics Conference, Vol. 2012-2849, New Orleans, LA, Jun. 2012. 
[33] Hannon, J. A., "1998/1999 Trap Wing Data Archive," http://hiliftpw.larc.nasa.gov/ 98-99WebArchiveWithMods/Trap\%20Wing\%20Data\%20Archive.html, 2014, [Online; retrieved 13July-2016].

[34] Horstmann, K.-H., "Ein Mehrfach-Traglinienverfahren und seine Verwendung für Entwurf und Nachrechnung nichtplanarer Flügelanordnungen.” DLR, German Aerospace Center, Rept. FB 87-51, 1987.

[35] Bramesfeld, G. and Maughmer, M. D., "Relaxed-wake Vortex-Lattice Method Using Distributed Vorticity Elements," Journal of Aircraft, Vol. 45, No. 2, 2008, pp. 560-568.

[36] Bissonnette, W., East, B., and Bramesfeld, G., "Design Optimization of the Flap System of a Large Transport Aircraft," CASI AERO 2015, Montreal, QC, 2015.

[37] Cole, J. A., Maughmer, M. D., and Bramesfeld, G., "Aerodynamic Design Considerations for Tiltrotor Wing Extensions and Winglets," 51st AIAA Aerospace Sciences Meeting, Vol. 2013-1088, Grapevine, TX, 2013.

[38] Combes, T. P., Malik, A. S., Bramesfeld, G., and McQuilling, M. W., "Efficient Fluid-Structure Interaction Method for Conceptual Design of Flexible, Fixed-Wing Micro-Air-Vehicle Wings," AIAA Journal, Vol. 53, No. 6, 2015, pp. 1442-1454.

[39] Bissonnette, W., "Development and Demonstration of a Conceptual Design Tool for Multiple Lifting Elements," Undergraduate Thesis, Ryerson University, 2014.

[40] Willis, D. J., An Unsteady, Accelerated, High Order Panel Method With Vortex Particle Wakes, Ph.D. thesis, Massachusetts Institute of Technology, 2006.

[41] Bissonnette, B. and Bramesfeld, G., "Effects of Wake Shapes on High-Lift System Aerodynamic Predictions," 54th AIAA Aerospace Sciences Meeting, Vol. 2016-2040, San Diego, CA, 2016.

[42] Sforza, P. M., Commercial Airplane Design Principles, Elsevier, Massachusetts, 2014.

[43] Olson, E. D. and Albertson, C. W., "Aircraft High-Lift Aerodynamic Analysis Using a SurfaceVorticity Solver," 54th AIAA Aerospace Sciences Meeting, Vol. 2016-0779, San Diego, CA, 2016. 
[44] Miller, S. G. and Youngblood, D. B., "Applications of USSAERO-B and the PANAIR Production Code to the CDAF Model, a Canard/Wing Configuration," 1983 AIAA Applied Aerodynamics Conference, Vol. 83-1829, Danvers, MA, 1983.

[45] Madson, M. D. and Erickson, L. L., "PAN AIR Analysis of the NASA/MCAIR 279-3: An advanced supersonic V/STOL fighter/attack aircraft," NASA TM-86838, Apr. 1986. 
BIBLIOGRAPHY 